\title{
INFERÊNCIA SOBRE MEDIDAS DE POSIÇÃO E DISPERSÃO EM DADOS CIRCULARES
}

\section{Gladys Dorotea Cacsire Barriga}

\author{
DISSERTAÇÃO APRESENTADA \\ $\mathrm{AO}$ \\ INSTITUTO DE MATEMÁTICA E ESTATÍSTICA \\ DA \\ UNIVERSIDADE DE SÃO PAULO \\ PARA OBTENÇÃO DO GRAU \\ DE \\ MESTRE EM ESTATÍSTICA
}

Área de Concentração: Estatística

Orientadora: Prof $\stackrel{a}{\text {. Dr }}$. Carmen Diva Saldiva de André

-São Paulo, maio de 1997- 


\title{
INFERENNCIA SOBRE MEDIDAS DE POSICÃO E DISPERSÃO EM DADOS CIRCULARES
}

\author{
Este exemplar corresponde à redação final \\ da dissertação devidamente corrigida e \\ defendida por Gladys Dorotea Cacsire Barriga \\ e aprovada pela comissão julgadora.
}

São Paulo, 23 maio de 1997.

Banca examinadora:

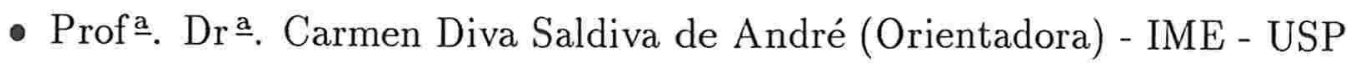

- Prof ‥ Dr ‥ Gilberto Alvarenga de Paula - IME - USP

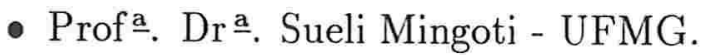




\title{
Resumo
}

Em muitos experimentos nas diversas áreas do conhecimento os dados correspondem a medidas de ângulos. A análise desses dados não pode ser feita utilizando-se as técnicas estatísticas usuais porque os resultados obtidos muitas vezes são incoerentes.

Nesta dissertação é apresentada parte da metodologia estatística para a análise de dados angulares. São apresentadas técnicas de análise descritiva, os modelos probabilísticos adotados com maior frequência e métodos de inferência sobre os parâmetros desses modelos em uma ou mais amostras independentes. São consideradas técnicas paramétricas e não paramétricas. Algumas aplicações ilustram a utilização das técnicas estudadas. Rotinas computacionais apropriadas são apresentadas no final do trabalho.

\begin{abstract}
In several experiments the data are measured in form of angles. The analysis of these data does not fit into the usual methods of statistical analysis.

Some statistical methods for the analysis of circular data are presented in this dissertation. Descriptive techniques, potential probabilistic models, and parametric and nonparametric inference methods for one or more independent samples are considered. Some applications illustrate the use of these techniques. At last, suitable computational routines are presented.
\end{abstract}




\section{Índice}

1 Introdução 1

2 Métodos descritivos 3

2.1 Introdução . . . . . . . . . . . . . . . . . . . . . . 3

2.2 Representação gráfica da amostra . . . . . . . . . . . . . . . . . 3

2.3 Medidas de locação . . . . . . . . . . . . . . . . . . . 4

2.3 .1 Direção média . . . . . . . . . . . . . . . . . . 5 5

2.3 .2 Direção mediana . . . . . . . . . . . . . . . . 10

2.3.3 Direção modal . . . . . . . . . . . . . . . . . . . 11

2.4 Medidas de dispersão na amostra . . . . . . . . . . . . . . 12

2.4.1 Variância amostral . . . . . . . . . . . . . . . 12

2.4.2 Desvio - padrão circular . . . . . . . . . . . . . . 14

2.4.3 Desvio médio circular . . . . . . . . . . . . . . . . . . . . . . 14

2.4 .4 Intervalo interquartílico . . . . . . . . . . . . . 15

2.4.5 Amplitude circular . . . . . . . . . . . . . . . 15

2.5 Momentos trigonométricos amostrais . . . . . . . . . . . . . . 16

2.6 Dispersão circular amostral e erro padrão circular . . . . . . . . . . . 18

2.7 Medidas de curtose e assimetria . . . . . . . . . . . . . . . 19

2.8 Modificações para dados axiais . . . . . . . . . . . . . . 20

$\begin{array}{lll}3 & \text { Modelos } & 21\end{array}$ 
3.1 Introdução . . . . . . . . . . . . . . . . . . . . . . 21

3.2 Momentos de distribuições circulares . . . . . . . . . . . . . . 22

3.3 Propriedades do vetor média e da variância circular . . . . . . . . 25

3.4 Distribuiçõos circulares . . . . . . . . . . . . . . . 25

3.4 .1 Distribuição uniforme . . . . . . . . . . . . 25

3.4 .2 Distribuição normal enrolada . . . . . . . . . . . . 26

3.4.3 Distribuição de von Mises . . . . . . . . . . . . . 27

4 Análise de uma amostra $\quad 34$

4.1 Introdução . . . . . . . . . . . . . . . . . . . . . 34

4.2 Verifição de uniformidade . . . . . . . . . . . . . . . . 35

4.2 .1 Método gráfico . . . . . . . . . . . . . . 35

4.2 .2 Teste de Rayleigh . . . . . . . . . . . . . . . . 35

4.3 Métodos não paramétricos para a análise de dados unimodais . . . 36

4.3 .1 Estimação da direção mediana . . . . . . . . . . . . 36

4.3.2 Teste de hipótese sobre a direção mediana . . . . . . . . . . 37

4.3 .3 Estimação da direção média . . . . . . . . . . . . . . . . 38

4.3.4 Teste de hipótese sobre a direção média . . . . . . . . . . . 38

4.4 Análise estatística de uma amostra da distribuição von Mises . . . . . 39

4.4 .1 Teste de aderência . . . . . . . . . . . . . . . 40

4.4 .2 Teste para outliers . . . . . . . . . . . . . . 41

4.4.3 Estimação de máxima verossimilhança dos parâmetros $\mu$ e $\kappa \quad 42$

4.4.4 Estimação por intervalo dos parâmetros $\mu$ e $\kappa \ldots . . . . .45$

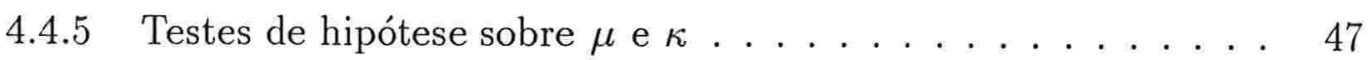

5 Técnicas não paramétricas para duas ou mais amostras 49

5.1 Introdução . . . . . . . . . . . . . . . . . . . . . . 49

5.2 Inferências sobre as direções medianas de duas ou mais populações circulares ........................ 51 
5.2.1 Teste de igualdade de duas ou mais direções medianas . . . . . 51

5.2.2 Estimação da direção mediana comum de duas ou mais populações 52

5.3 Inferências sobre as direções médias de duas ou mais populações circulares . . . . . . . . . . . . . . . . 52

5.3.1 Teste da igualdade de duas ou mais direções médias . . . . . . 53

5.3.2 Estimação da direção média comum de duas ou mais distribuições 55

5.4 Teste de igualdade das dispersões de duas ou mais distribuições . . . 56

5.5 Teste de igualdade de duas ou mais distribuições . . . . . . . . . . . 58

5.5.1 O teste dos escores uniformes . . . . . . . . . . . 59

5.6 Generalização para dados axias . . . . . . . . . . . . . 62

6 Análise de duas ou mais amostras da distribuição de von Mises $\quad 63$

6.1 Introdução . . . . . . . . . . . . . . . . . 63

6.2 Testes de igualdade de direções médias . . . . . . . . . . . . . 63

6.2.1 Os parâmetros de concentração não são todos iguais . . . . . . 64

6.2 .2 Os parâmetros de concentração são iguais . . . . . . . . . 65

6.3 Inferências após a comparação das direções médias . . . . . . . . . . 68

6.3.1 Estimação da direção média comum de duas ou mais distribuições de von Mises . . . . . . . . . . . 68

6.3.2 Construção de intervalos de confiança para a diferença de duas direções médias . . . . . . . . . . . . . . . 69

6.4 Teste de igualdade de parâmetros de concentração de duas ou mais distribuições de von Mises . . . . . . . . . . . . . . . . . 71

6.5 Estimação do parâmetro de concentração comum de duas ou mais distribuições de von Mises . . . . . . . . . . . . . . . 73

7 Aplicações $\quad 75$

7.1 Introdução . . . . . . . . . . . . . . . . . 75

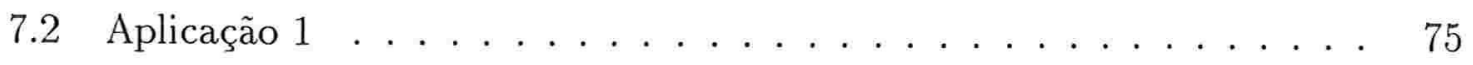

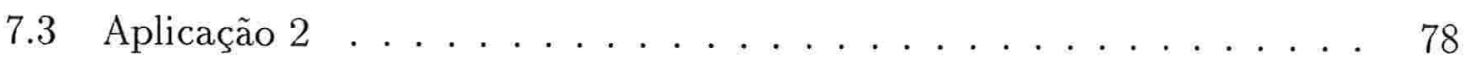


7.4 Aplicação $3 \ldots \ldots$. . . . . . . . . . . . . . . . . 80

8 Comentários finais $\quad 83$

$\begin{array}{ll}\text { Apêndices } & 85\end{array}$

A Demostração de propriedades do vetor média $\quad 85$

B Métodos Bootstrap $\quad 88$

B.1 Introdução . . . . . . . . . . . . . . . . . . . . . . . . . . 88

B.2 Intervalo de confiança para a direção média $\mu \ldots \ldots$. . . . . . . 89

B.3 Teste de hipótese sobre a direção média $\mu$ em problemas com uma amostra . . . . . . . . . . . . . . . 90

B.4 Comparação das direções médias de duas ou mais populações . . . . . 90

B.5 Estimação da direção média comum de duas ou mais distribuições . . 91

B.6 Intervalo de confiança para a direção média $\mu$ de uma $V M(\mu, \kappa)$. . . 92

B.7 Teste de hipótese sobre a direção média de uma população $\operatorname{VM}(\mu, \kappa) \quad 92$

B.8 Intervalo de confiança para o parâmetro de concentração em uma $V M(\mu, \kappa) \ldots \ldots \ldots \ldots \ldots \ldots$

B.9 Teste de hipótese sobre a o parâmetro de concentração em uma $V M(\mu, \kappa)$ 93

B.10 Teste de igualdade de direções médias de duas ou mais distribuições von Mises . . . . . . . . . . . . . . . . . . . . . 93

B.11 Estimação da direção média comun de 2 ou mais distribuições von Mises 93

B.12 Estimação do parâmetro de concentração comum de duas ou mais distribuições de von Mises . . . . . . . . . . . . . . . . . . . . . . . . . 94

$\begin{array}{ll}\text { C Programas } & 95\end{array}$

Referências Bibliográficas $\quad 122$ 


\section{Capítulo 1}

\section{Introdução}

Medidas angulares ocorrem em muitas ciências aplicadas tais como biologia, medicina, geografia, meteorologia, geofísica, oceanografia, etc. Exemplos típicos de tais dados incluem direções de partida e de desaparecimento de pássaros em pontos de soltura, movimento direcional de animais em reposta a um estímulo, direções do vento e correntes de oceanos, direções de seixos em leitos de rio e direções de fraturas em rochas. Também resultados de estudos de um fenômeno periódico, com um período conhecido, podem ser encarados como dados circulares. Por exemplo, aos horários de chegada de pacientes à UTI de um hospital em um período de $24 \mathrm{~h}$ podemos fazer corresponder ângulos, considerando um "relógio"de $24 \mathrm{~h}$, isto é, a cada grau associamos um intervalo de tempo de 4 minutos.

A periodicidade inerente aos dados circulares traz consigo problemas de natureza peculiar que não ocorrem em observações na reta. Este fato faz com que sejam necessárias definições específicas de medidas de posição e dispersão, bem como de momentos, e sejam considerados modelos probabilísticos apropriados.

A literatura básica sobre este assunto consiste em Mardia(1972), Batschelet(1981) e Fisher(1993). Nos útimos dez anos observou-se grande desenvolvimento dos métodos de análise de dados circulares. Em particular, métodos baseados na técnica "bootstrap"foram propostos para a análise de pequenas amostras.

Podemos dividir as observações angulares em duas grandes categorias. Dizemos que os dados são vetoriais, quando pudermos atribuir um sentido à direção observada. Como exemplo, temos: direções de deslocamento de um animal, direções do vento, horário de chegada de pacientes à UTI de um hospital, etc. Os dados são denominados axiais, caso contrário. Por exemplo, orientações de montes de cupim, orientações de fraturas em uma rocha, etc... . Efetivamente, a amplitude desse tipo de observação 
pode ser reduzida a $180^{\circ}$.

O objetivo deste trabalho é apresentar, de forma clara, técnicas estatísticas para inferências sobre as medidas de posição e dispersão em uma ou mais amostras independentes de dados circulares, e implementá-las em rotinas computacionais. Restringiremos nosso estudo a amostras de dados vetoriais, indicando brevemente como algumas técnicas podem ser aplicadas a dados axiais.

No capítulo 2 mostramos como resumir de forma descritiva as informações contidas em uma amostra. No capítulo 3 apresentamos os modelos probabilísticos utilizados com maior frequência na literatura de dados circulares. No capítulo 4 consideramos métodos de análise para uma amostra de uma população circular. Técnicas nãoparamétricas para análise de duas ou mais amostras são apresentadas no capítulo 5 . No capítulo 6 é apresentada a análise de duas ou mais amostras da distribuição de von Mises. Com o objetivo de ilustrar as técnicas descritas no trabalho, no capítulo 7 apresentamos algumas aplicações numéricas. 


\section{Capítulo 2}

\section{Métodos descritivos}

\subsection{Introdução}

Neste capítulo trataremos da análise exploratória de dados circulares. Como veremos, as técnicas de descrição e resumo de uma amostra $\theta_{1}, \ldots, \theta_{n}$ de observações angulares são bastante diferentes das utilizadas para dados na reta. Apresentaremos formas de representação gráfica das observações e o cálculo de medidas descritivas de posição, dispersão, curtose e assimetria.

\subsection{Representação gráfica da amostra}

A representação gráfica da amostra é feita associando-se a cada ângulo observado um ponto sobre uma circunferência. Ilustramos esse procedimento nos exemplos 2.2.1 e 2.2.2. Observemos que a construção desses gráficos nos permite ter uma idéia inicial sobre características importantes da amostra tais como : se a amostra provém de uma distribuição uniforme(ver capítulo 3), se ocorrem uma ou mais modas, se a distribuição é simétrica, e se ocorreram valores aberrantes ( "outliers"). Quando os dados são axiais, costuma-se associar a cada ângulo da amostra um ponto em cada extremo do eixo que passa pelo centro da circunferência e tem direção igual ao ângulo observado. 
Exemplo 2.2.1 (Mardia, 1972) Uma roleta foi girada 9 vêzes e suas posições de parada foram medidas, considerando-se os ângulos em relação a uma direção fịxa. Os resultados obtidos, em graus, foram: 43,45,52,61,75, 88,88, 279, 357 e estão representados na Figura 2.1.

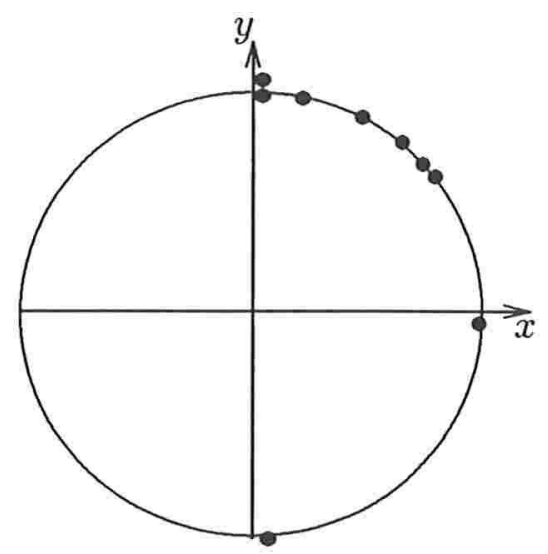

Figura 2.1: Representação das posições de parada de uma roleta.

Observamos que existe uma "direção de preferência", pois os dados se concentram no arco $\left(0^{\circ}, 90^{\circ}\right)$.

Exemplo 2.2.2 (Batschelet, 1981) Os rumos, em graus, tomados por 15 pomboscorreio, quando soltos individualmente a $66 \mathrm{~km}$ de seu pombal, foram: 250, 275, 285, $285,290,290,295,300,305,310,315,320,330,330,5$.

A representação gráfica da amostra está na Figura 2.2 .

\subsection{Medidas de locação}

Nesta seção apresentaremos métodos de obtenção de medidas de locação em uma amostra unimodal de dados vetoriais. O cálculo dessas medidas para dados axiais se efetuará como descrito no final do capítulo.

Denotaremos por $P_{i}$ o ponto correspondente ao ângulo $\theta_{i}$ numa circunferência centrada na origem, com raio unitário. A cada ponto $P_{i}$ corresponde um vetor unitário $\overrightarrow{O P}_{i}, i=1, \ldots, n$ (ver Figura 2.3). Considerando um sistema cartesiano com origem em $O$, as coordenadas cartesianas de $P_{i}$ são $\left(\cos \theta_{i}, \operatorname{sen} \theta_{i}\right), i=1, \ldots, n$. 


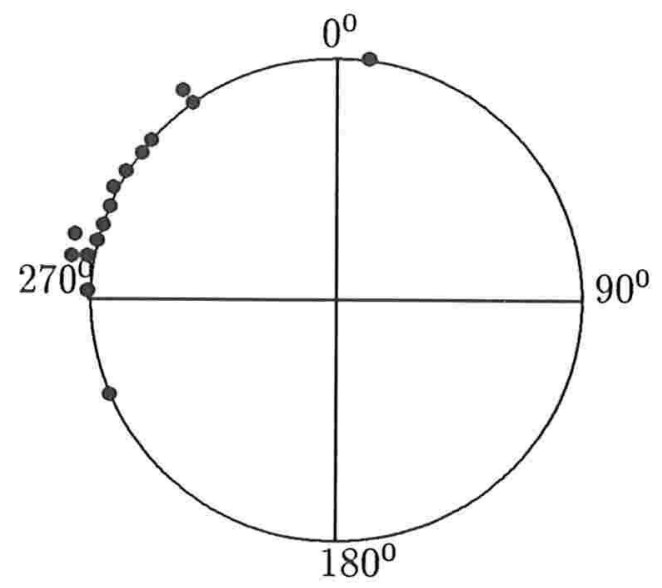

Figura 2.2: Representação dos rumos de 15 pombos correio.

\subsubsection{Direção média}

Para ilustrar o problema do cálculo da direção média em dados circulares, consideremos uma amostra de três direções dadas pelos ângulos: $\theta_{1}=80^{\circ}, \theta_{2}=350^{\circ}$, e $\theta_{3}=50^{\circ}$; observando a representação gráfica desses valores dada na Figura 2.4, é de se esperar que o ângulo médio assuma um valor entre $0^{0}$ e $50^{\circ}$. Entretanto, se calcularmos a média aritmética dos valores abservados, obtemos que $\left(\theta_{1}+\theta_{2}+\theta_{3}\right) / 3=160^{\circ}$, que é claramente um valor inaceitável.

Esse exemplò simples mostra que a média aritmética dos ângulos da amostra pode não ser uma estatística adequada para representar a direção média.

Consideremos, então, a representação gráfica dos ângulos da amostra num círculo unitário dada na Figura 2.4. A cada ponto podemos associar massa de igual valor, $M$, e encontrarmos o centro de massa, $G$, também chamado centro de gravidade (ver Figura 2.5). Se este centro é diferente da origem $O$, então o vetor que aponta para o centro de gravidade $\overrightarrow{O G}$ é definido como o vetor média amostral, e determina uma direção denominada direção média amostral (ou ângulo médio). Na Figura 2.5 representamos o centro de gravidade e a direção média em uma amostra de tamanho três.

Vamos considerar duas formas de determinação do vetor média: uma utiliza álgebra vetorial e a outra funções trigonométricas. 


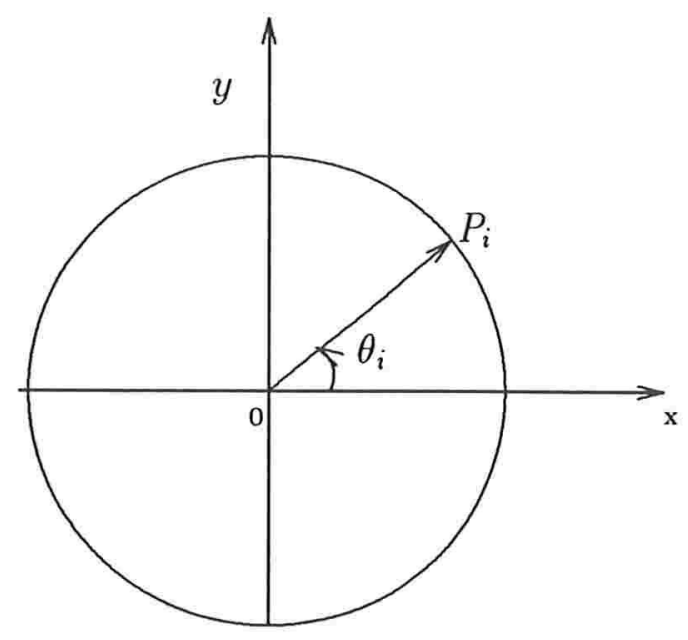

Figura 2.3: Representação gráfica do $i$-ésimo ponto amostral $\theta_{i}$.

Determinação do vetor média através de álgebra vetorial

Sejam $\overrightarrow{O P}_{1}, \overrightarrow{O P}_{2}, \ldots, \overrightarrow{O P}_{n}$, os vetores unitários correspondentes à amostra $\theta_{1}, \theta_{2}, \ldots, \theta_{n}$. Atribuindo massa $M$ a $P_{1}, P_{2}, \ldots, P_{n}$, temos que o vetor:

$$
\overrightarrow{O G}=\frac{1}{n} \sum_{i=1}^{n} \overrightarrow{O P}_{i}
$$

aponta para o centro de massa de $P_{1}, P_{2}, \ldots \ldots, P_{n}$. Então $\overrightarrow{O G}$ é o vetor média da amostra.

Denotamos por $R$ o comprimento do vetor resultante, isto é,

$$
R=\left\|\sum_{i=1}^{n} \overrightarrow{O P}_{i}\right\|
$$

e, por $r$ o comprimento do vetor média, ou seja,

$$
r=\|\overrightarrow{O G}\|=\frac{R}{n}
$$

Observemos que o centro de massa $G$ se localiza, em geral, no interior da circunferência de raio unitário. Ele será um ponto da circunferência somente no caso especial em que todos os ângulos da amostra são iguais, ou seja, as massas ocorrem 


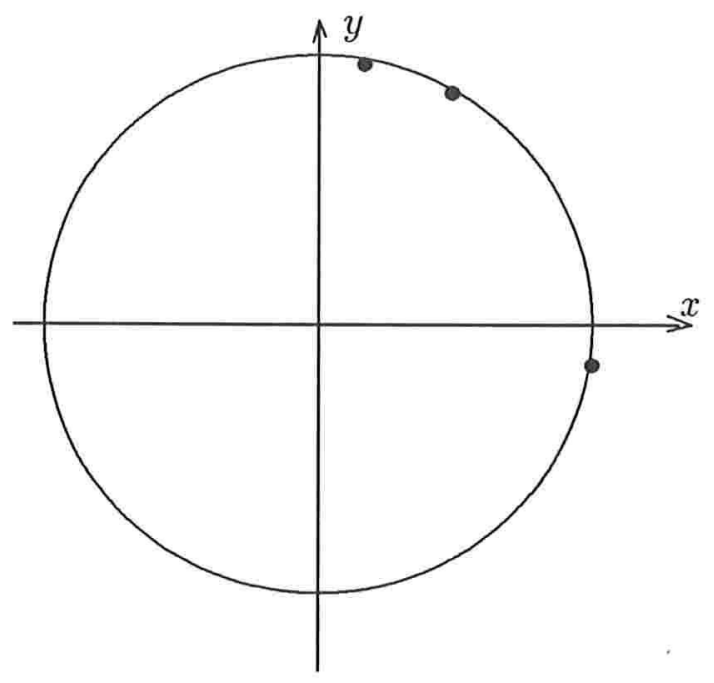

Figura 2.4: Representação gráfica de uma amostra.

em um único ponto. Como estamos considerando vetores de comprimento unitário, temos ainda que,

$$
0 \leq R \leq n \quad \text { e } \quad 0 \leq r \leq 1
$$

\section{Determinação do vetor média utilizando funções trigonométricas}

Denotemos por $(\bar{x}, \bar{y})$ as coordenadas cartesianas do centro de massa $G$ de $P_{1}, \ldots, P_{n}$, ou seja, se $P_{i}=\left(x_{i}, y_{i}\right), i=1,2, \ldots, n$,

$$
\bar{x}=\frac{1}{n} \sum_{i=1}^{n} x_{i}, \quad \text { e } \quad \bar{y}=\frac{1}{n} \sum_{i=1}^{n} y_{i} .
$$

Podemos também escrever as coordenadas de $G$ como:

$$
\bar{x}=\frac{1}{n} \sum_{i=1}^{n} \cos \theta_{i}=\bar{C} \quad \text { e } \quad \bar{y}=\frac{1}{n} \sum_{i=1}^{n} \operatorname{sen} \theta_{i}=\bar{S} .
$$




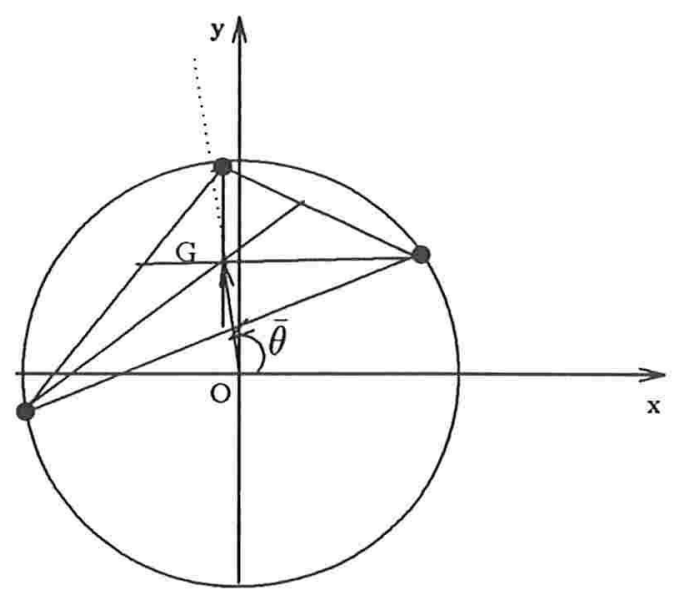

Figura 2.5: Representação gráfica do centro de gravidade em uma amostra.

Ou seja, as coordenadas cartesianas do centro de massa $G$ podem ser escritas como $(\bar{C}, \bar{S})$. O comprimento do vetor média $\overrightarrow{O G}$ é, então:

$$
r=\left[\bar{C}^{2}+\bar{S}^{2}\right]^{1 / 2}=\frac{1}{n}\left[\left(\sum_{i=1}^{n} \cos \theta_{i}\right)^{2}+\left(\sum_{i=1}^{n} \operatorname{sen} \theta_{i}\right)^{2}\right]^{1 / 2}
$$

Então:

$$
R=\left[\left(\sum_{i=1}^{n} \cos \theta_{i}\right)^{2}+\left(\sum_{i=1}^{n} \operatorname{sen} \theta_{i}\right)^{2}\right]^{1 / 2} .
$$

Da definição de direção média e de (2.1), temos então que a direção média amostral $\bar{\theta}$ é dada por:

$$
\bar{\theta}= \begin{cases}\operatorname{arctag}\left(\frac{\bar{S}}{\bar{C}}\right) & \text { se } \bar{S}>0, \bar{C}>0 \\ 180^{\circ}+\operatorname{arctag}\left(\frac{\bar{S}}{\bar{C}}\right) & \text { se } \bar{C}<0 ; \\ 360^{\circ}+\operatorname{arctag}\left(\frac{\bar{S}}{\bar{C}}\right) & \text { se } \bar{S}<0, \bar{C}>0 .\end{cases}
$$

São casos excepcionais:

$$
\bar{\theta}= \begin{cases}90^{\circ} & \text { se } \bar{S}>0, \bar{C}=0 \\ 270^{\circ} & \text { se } \bar{C}=0 \quad \bar{S}<0 \\ \text { não determinado } & \text { se } \bar{S}=0, \bar{C}=0\end{cases}
$$


Exemplo 2.3.1 Consideremos os dados do exemplo 2.2.1. Temos que $\sum_{i=1}^{n} \cos \theta_{i}=$ 4,0226; $\sum_{i=1}^{n} \operatorname{sen} \theta_{i}=4,9764 ; \bar{C}=0,4470 ; \bar{S}=0,5529$. Então $\bar{\theta}=\operatorname{arctag}\left(\frac{\bar{S}}{\bar{C}}\right)=51,0^{0}$.

Exemplo 2.3.2 Consideremos os dados do exemplo 2.2.2. Obtemos: $\sum_{i=1}^{n} \cos \theta_{i}=$ 7,$287 ; \sum_{i=1}^{n} \operatorname{sen} \theta_{i}=-11,367 ; \bar{C}=0,4858 ; \quad \bar{S}=-0,7578, \quad r=0,9001 . \quad$ Como $\bar{S}<0$ e $\bar{C}>0$, temos: $\bar{\theta}=360^{\circ}+\operatorname{arctag}\left(\frac{\bar{S}}{\bar{C}}\right)=302,7^{0}$.

\section{Propriedades do vetor média}

O vetor média $\overrightarrow{O G}$ e, consequentemente, a direção média $\bar{\theta}$, possuem as seguintes propriedades que estão demostradas no Apêndice $A$ :

1: o vetor média não depende da origem, isto é, permanece na mesma posição se mudarmos a direção correspondente a zero graus. Suponhamos que a direção do ângulo zero seja rotacionada em um ângulo $\alpha$ no sentido anti-horário. Então os valores amostrais $\theta_{i}$ passam a ser $\theta_{i}^{\prime}=\theta_{i}-\alpha, i=1,2, \ldots, n$. O novo ângulo médio passa a ser $\bar{\theta}^{\prime}=\bar{\theta}-\alpha$, e o comprimento do vetor média $r$ não se altera.

2: $\sum_{i=1}^{n} \operatorname{sen}\left(\theta_{i}-\bar{\theta}\right)=0$. Em estatística circular, desvios das observações $\theta_{i}$ em relação à direção média são medidos por $\operatorname{sen}\left(\theta_{i}-\bar{\theta}\right)$ e $\cos \left(\theta_{i}-\bar{\theta}\right), i=1, \ldots, n$. Notemos que a soma do desvios em torno da média: $\sum_{i=1}^{n} \operatorname{sen}\left(\theta_{i}-\bar{\theta}\right)=0 \mathrm{em}$ analogia ao caso linear: $\sum_{i=1}^{n}\left(x_{i}-\bar{x}\right)=0$.

3: $\sum_{i=1}^{n} \cos \left(\theta_{i}-\bar{\theta}\right)=n r$

4: $\frac{1}{n} \sum_{i=1}^{n}\left(\theta_{i}-\bar{\theta}\right)^{2} \approx 2(1-r)$, se os desvios $\left(\theta_{i}-\bar{\theta}\right)$ forem pequenos, $i=1, \ldots, n$. 


\subsubsection{Direção mediana}

Consideremos a representação da amostra $\theta_{1}, \ldots, \theta_{n}$ sobre o círculo unitário centrado em $O$ dada na Figura 2.6. Um segmento $O P$, onde $P$ é um ponto da circunferência, com as seguintes propriedades:

i) Metade dos pontos amostrais estão de cada lado do diâmetro $P Q$ e

ii) A maioria dos pontos amostrais são mais próximos de $P$ do que de $Q$

define a direção mediana da amostra.

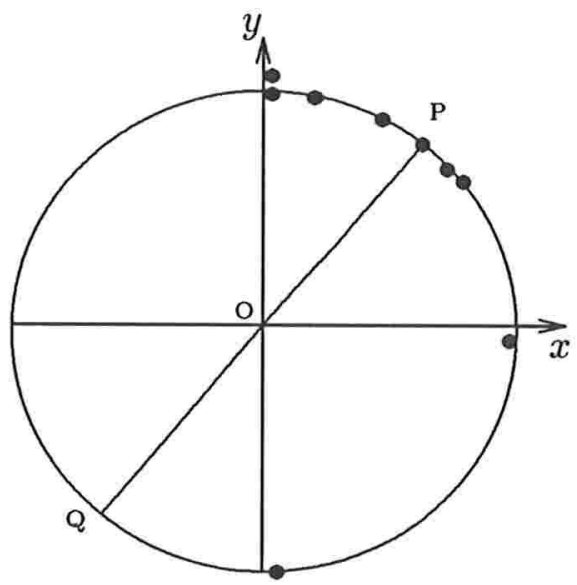

Figura 2.6: Representação da direção mediana.

Se o tamanho da amostra $n$ é impar, $P$ é um dos pontos da amostra; quando $n$ é par $P$ está entre dois pontos amostrais.

Observemos que, se os dados estão concentrados em um arco de comprimento substancialmente menor que a circunferência toda (menor que $180^{\circ}$ ), o procedimento para a determinação da mediana em dados lineares pode ser adotado. Neste caso, quando o conjunto de pontos ultrapassa $0^{\circ}$, isto é, ocorrem observações à direita e à esquerda de $0^{0}$ (por exemplo, $10^{0}, 5^{0}, 1^{0}, 358^{0}, 350^{\circ}$ ), deve-se inicialmente fazer uma rotação de forma que isto não ocorra, (no exemplo, fazendo uma rotação de $10^{\circ}$ no sentido horário, as observações passam a ser,respectivamente: $20^{\circ}, 15^{0}, 11^{0}, 8^{0}$ e $0^{0}$ ). Aplica-se o procedimento para determinação da mediana na reta, e depois é feita a mesma rotação no sentido oposto. 
A mediana também pode ser determinada minimizando-se a expressão:

$$
d(\theta)=\pi-\frac{1}{n} \sum_{i=1}^{n}|\pi-| \theta_{i}-\theta||
$$

Exemplo 2.3.3 Consideremos os dados do Exemplo 2.2.1. Se considerássemos as observações como dados na reta, a direção mediana seria $75^{\circ}$. Entretanto, o diâmetro que passa pelo ponto correspondente a $75^{\circ}$ não divide a circunferência em duas partes com igual número de observações, como podemos ver na Figura 2.1. O ângulo com as propriedades $i$ ) e ii) dadas acima corresponde a $\tilde{\theta}=52^{0}$.

Uma outra forma de se chegar a este resultado é observar que todos os dados estão concentrados em um arco de comprimento menor que $180^{\circ}$. Neste exemplo podemos rotacionar os eixos em $90^{\circ}$ no sentido horário. Desta forma, todos os valores observados estarão entre $0^{\circ}$ e $180^{\circ}$, assumindo valores $\theta_{i}^{\prime}=\left(\theta_{i}+90^{\circ}\right)\left(\bmod 360^{\circ}\right)$, ou seja $, 9^{0}, 87^{\circ}, 133^{\circ}, 135^{\circ}, 142^{0}, 151^{\circ}, 165^{\circ}, 178^{\circ}, 178^{\circ}$. Utilizando o mesmo procedimento que em dados na reta, temos que a mediana é $142^{\circ}$. Fazendo agora a rotação no sentido inverso, obtemos que $\tilde{\theta}=142^{\circ}-90^{\circ}=52^{\circ}$.

Exemplo 2.3.4 Consideremos os dados do exemplo 2.2.2. O diâmetro pelo ponto correspondente a $300^{\circ}$ divide a circunferência em duas partes com igual número de pontos, e, portanto, $\tilde{\theta}=300^{\circ}$ é a direção mediana.

De outra forma, após rotacionar os eixos em $180^{\circ}$ no sentido horário obtemos os ângulos: $70^{\circ}, 95^{\circ}, 105^{\circ}, 105^{\circ}, 110^{\circ}, 110^{\circ}, 115^{\circ}, 120^{\circ}, 125^{\circ}, 130^{\circ}, 135^{\circ}, 140^{\circ}, 150^{\circ}$, $150^{\circ}, 180^{\circ}$. Calculando-se a mediana como em dados na reta obtemos o ângulo $120^{\circ}$. Rotacionando no sentido inverso obtemos que $\tilde{\theta}=\left(120^{\circ}-180^{\circ}\right) \quad\left(\bmod 360^{\circ}\right)=$ $300^{0}$.

\subsubsection{Direção modal}

A direção modal $\ddot{\theta}$ na amostra é a direção de concentração máxima dos dados. Sua determinação é análoga ao caso na reta.

Exemplo 2.3.5 Consideremos os dados do Exemplo 2.2.1. A direção modal é dada por: $\check{\theta}=88^{\circ}$. 


\subsection{Medidas de dispersão na amostra}

Considerando amostras de dados vetoriais unimodais apresentamos nesta seção algumas medidas de concentração desses ângulos em torno da direção média. No final do capítulo trataremos do cálculo de medidas de dispersão para dados axiais. Uma outra medida de dispersão (dispersão circular amostral) obtida a partir dos momentos trigonométricos amostrais a serem definidos, será apresentada na seção 2.5.

\subsubsection{Variância amostral}

Na Figura 2.7 representamos graficamente 4 amostras de tamanho 5, com o mesmo ângulo médio. Observamos que à medida que aumenta a dispersão dos pontos, diminui o comprimento do vetor média $(r)$. Como $0 \leq r \leq 1$, notamos que os valores extremos de $r$ possuem propriedades interessantes: $r=1$ implica que todos os pontos são coincidentes e $r=0$ implica em uma dispersão uniforme pelo círculo. Assim, em dados unimodais, o comprimento do vetor média pode ser considerado como uma medida de concentração em torno do ângulo médio. A variância circular amostral é, então, definida como:

$$
V=1-r
$$

Notemos que $0 \leq V \leq 1$, o que não ocorre em dados na reta. Quanto menor o valor de $V$, mais homogênea é a amostra.

Utilizando argumentos diferentes, Mardia(1972) demonstrou que a expressão (2.4) é uma medida de variabilidade em torno do ângulo médio. Seja $P_{i}$ um ponto correspondente ao ângulo $\theta_{i}$ em um círculo de raio unitário e $P$ o ponto correspondente a uma direção $\alpha$ fixada. Inicialmente vamos supor $\alpha=0$. Uma medida de dispersão de $P_{i}$ em relação a $P$ é o menor dos dois ângulos que o segmento $O P_{i}$ forma com $O P$. Denotemos esse ângulo por $\varepsilon_{i}$. Assim:

$$
\varepsilon_{i}=\min \left(\theta_{i}, 2 \pi-\theta_{i}\right)=\pi-\left|\left(\pi-\theta_{i}\right)\right| .
$$

Notemos que $0^{0} \leq \varepsilon_{i} \leq 180^{\circ}$. Como $1-\cos \varepsilon_{i}$ é uma função crescente monótona de $\varepsilon_{i}$

$$
D=\frac{1}{n} \sum_{i=1}^{n}\left(1-\cos \varepsilon_{i}\right)
$$

é uma medida de dispersão dos pontos $P_{i}$ correspondentes a $\theta_{i}, i=1, \ldots, n$. Mas: 

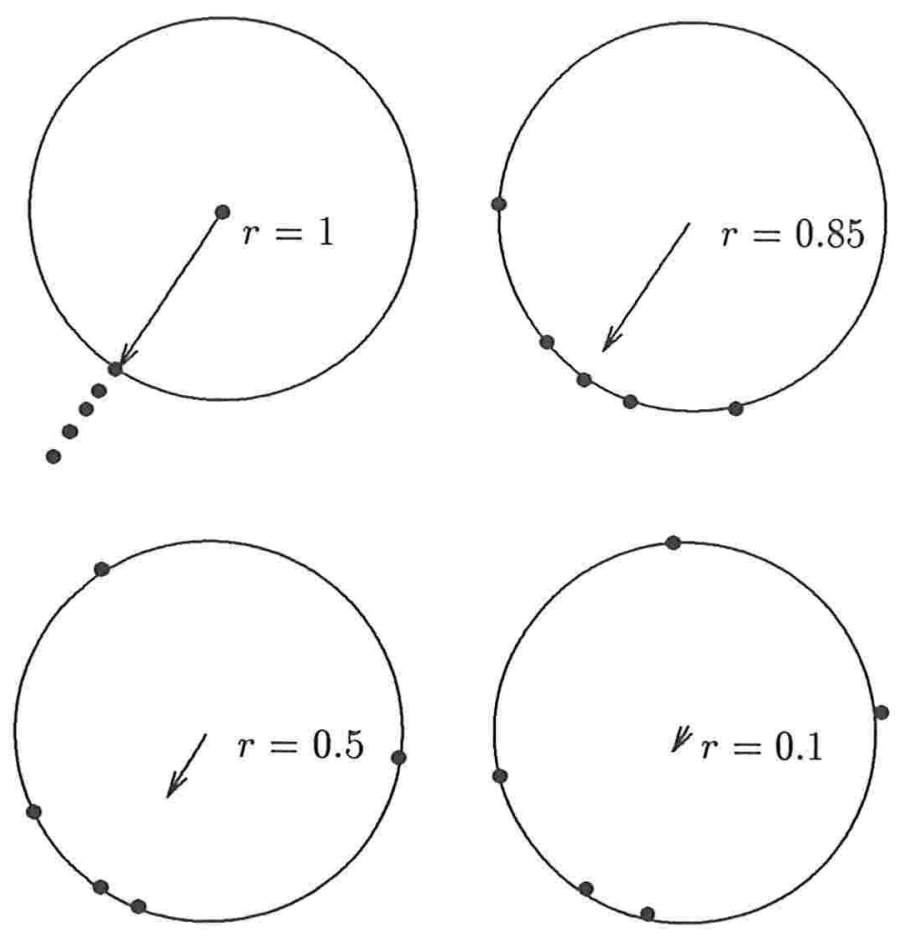

Figura 2.7: Vetor média em quatro amostras de dados circulares.

$$
\frac{1}{n} \sum_{i=1}^{n}\left(1-\cos \varepsilon_{i}\right)=\frac{1}{n} \sum_{i=1}^{n}\left(1-\cos \theta_{i}\right) .
$$

Num caso geral, considerando a direção zero igual a $\alpha$ e fazendo $\theta_{i}^{\prime}=\theta_{i}-\alpha$, temos:

$$
\begin{aligned}
D & =\frac{1}{n} \sum_{i=1}^{n}\left(1-\cos \left(\theta_{i}-\alpha\right)\right) \\
& =1-\frac{1}{n} \sum_{i=1}^{n} \cos \left(\theta_{i}-\alpha\right) \\
& =1-r \cos (\bar{\theta}-\alpha) .
\end{aligned}
$$

Assim, se considerarmos a direção zero $\alpha=\bar{\theta}$, temos que:

$$
D=1-r
$$


Observemos que, como o comprimento do vetor média não é afetado por uma mudança da origem, (subseção 2.3.1, propriedade (2))a variância circular também não o será.

\subsubsection{Desvio - padrão circular}

O desvio - padrão circular amostral é definido como:

$$
v=[-2 \ln (1-V)]^{1 / 2},
$$

onde $V$ é a variância circular. Notemos que, enquanto $0 \leq V \leq 1, v$ assume valores no intervalo $[0, \infty)$. Temos também que o desvio - padrão circular não é definido como a raiz quadrada da variância circular.

Uma aproximação para $v$ é dada por: $v \simeq(2 V)^{1 / 2}$, pois $V \simeq \ln (1-V)$ para valores pequenos de $V$.

O erro nesta aproximação é menor que $5 \%$ para $V<0,18$, ou seja $r>0,82$, como discutido por Fisher(1993).

\subsubsection{Desvio médio circular}

Seja $\theta_{1}, \ldots, \theta_{n}$ uma amostra de dados circulares. Rotacionando a direção zero no sentido anti-horário em um ângulo $\alpha$, podemos escrever $\theta_{i}^{\prime}=\left(\theta_{i}-\alpha\right) \quad(\bmod 2 \pi)$. Na subseção 2.4 .1 consideramos que $\varepsilon_{i}$ é dado por:

$$
\varepsilon_{i}=\min \left(\theta_{i}^{\prime}, 2 \pi-\theta_{i}^{\prime}\right)=\pi-\left|\pi-\theta_{i}\right|,
$$

é uma medida de desvio de $\theta_{i}^{\prime}$ em relação a $\alpha$. Este desvio pode ser considerado como uma distância circular e podemos utilizá-lo para definir a seguinte medida de dispersão:

$$
d_{0}=\frac{1}{n} \sum_{i=1}^{n} \varepsilon_{i}
$$

denominada desvio circular médio em relação à direção $\alpha$. Podemos também escrever:

$$
d_{0}=\pi-\frac{1}{n} \sum_{i=1}^{n}\left|\pi-\theta_{i}^{\prime}\right|=\pi-\frac{1}{n} \sum_{i=1}^{n}|\pi-| \theta_{i}-\alpha|| .
$$


Mardia(1972) mostrou que $d_{0}$ assume valor mínimo quando $\alpha$ é igual à direção mediana $\tilde{\theta}$.

\subsubsection{Intervalo interquartílico}

Definimos a primeira direção quartílica como sendo o ângulo $Q_{1}^{0}$ tal que $25 \%$ das observações pertencem ao arco determinado pela direção mediana $\theta$ e $Q_{1}^{0}$, quando percorremos a circunferência no sentido horário.

A terceira direção quartílica $Q_{3}^{0}$ é tal que $25 \%$ das observações estão localizadas entre $\tilde{\theta}$ e $Q_{3}^{0}$, quando percorremos a circunferência no sentido anti-horário.

O intervalo interquartílico é o comprimento do arco $\left(Q_{1}^{0}, Q_{3}^{0}\right)$ que contém $\tilde{\theta}$.

Exemplo 2.4.1 Considerando os dados do exemplo 2.2.1. Obtemos:

$$
\begin{aligned}
& Q_{1}^{0}=26^{0} \\
& Q_{3}^{0}=81,5^{0} .
\end{aligned}
$$

Logo, o intervalo interquartílico é $55,5^{0}$.

\subsubsection{Amplitude circular}

A amplitude de uma amostra circular é o comprimento do menor arco que contém todos os pontos amostrais.

Para a determinação da amplitude precisamos da definição das estatísticas de ordem circulares. Consideremos uma amostra de $n$ observações $\theta_{1}, \ldots, \theta_{n}$, onde $0<$ $\theta_{i} \leq 2 \pi$, e a correspondente amostra ordenada em ordem crescente $\theta_{(1)} \leq \ldots \leq \theta_{(n)}$. $\theta_{(i)}$ é denominada a i-ésima estatística de ordem. Notemos que a ordenação da amostra é afetada pela mudança da direção zero. Para a determinação da amplitude definimos:

$$
\begin{aligned}
T_{i} & =\theta_{(i+1)}-\theta_{(i)}, \quad i=1, \ldots, n-1, \quad e \\
T_{n} & =2 \pi-\theta_{(n)}+\theta_{(1)},
\end{aligned}
$$

onde $T_{i}, i=1, \ldots, n$ são medidas dos comprimentos de arco entre pontos consecutivos. A amplitude circular é definida por: 


$$
W=2 \pi-\max \left(T_{1}, \ldots, T_{n}\right)
$$

Exemplo 2.4.2 Considerando os dados do Exemplo 2.2.1. Obtemos:

$$
\begin{aligned}
& \theta_{(i)}: 43,45,52,61,75,88,88,279,357 \\
& T_{i}: 2,7,9,14,13,13,191,78,46 .
\end{aligned}
$$

Logo, a amplitude é:

$$
W=360^{\circ}-191^{\circ}=169^{\circ} .
$$

\subsection{Momentos trigonométricos amostrais}

Na seção 2.3 vimos que a média aritmética não é adequada como medida de posição em dados circulares. No geral, os momentos amostrais em distribuições na reta não são aplicáveis a distribuições circulares, e necessitamos da definição dos momentos trigonométricos amostrais que vão ser constituídos por vetores de dimensão $2 \times 1$.

O vetor média $\overrightarrow{O G}$ definido na subseção 2.3.1, é denominado primeiro momento trigonométrico amostral. Podemos representá-lo utilizando suas coordenadas cartesianas, ou seja,

$$
\overrightarrow{O G}=\mathbf{m}_{1}^{\prime}=(\bar{C}, \bar{S})
$$

onde

$$
\bar{C}=\frac{1}{n} \sum_{i=1}^{n} \cos \theta_{i}
$$

$\mathrm{e}$

$$
\bar{S}=\frac{1}{n} \sum_{i=1}^{n} \operatorname{sen} \theta_{i}
$$

ou, em cordenadas polares,

$$
\mathrm{m}_{1}^{\prime}=(r, \bar{\theta}) .
$$


Utilizando a notação de números complexos, podemos também escrever:

$$
\mathrm{m}_{1}^{\prime}=\bar{C}+i \bar{S}=r(\cos \bar{\theta}+i \operatorname{sen} \bar{\theta})=r e^{i \bar{\theta}} .
$$

O segundo momento trigonométrico amostral é definido como:

$$
\mathbf{m}_{2}^{\prime}=\left(\bar{C}_{2}, \bar{S}_{2}\right)
$$

onde:

$$
\begin{aligned}
& \bar{C}_{2}=\frac{1}{n} \sum_{i=1}^{n} \cos 2 \theta_{i} e \\
& \bar{S}_{2}=\frac{1}{n} \sum_{i=1}^{n} \operatorname{sen} 2 \theta_{i},
\end{aligned}
$$

podendo também ser escrito como:

$$
\mathbf{m}_{2}^{\prime}=\bar{C}_{2}+i \bar{S}_{2}=r_{2} e^{i \bar{\theta}_{2}}
$$

onde

$$
\begin{aligned}
& r_{2}=\left(\bar{C}_{2}{ }^{2}+\bar{S}_{2}{ }^{2}\right)^{1 / 2} e \\
& \bar{\theta}_{2}: \text { direção do segundo momento amostral. }
\end{aligned}
$$

Notemos que, para obter o vetor $\mathbf{m}_{2}^{\prime}$, primeiro duplicamos cada ângulo, ou seja, consideramos os ângulos $2 \theta_{i}(\bmod 2 \pi)$ ao invés de $\theta_{i}$. A nova distribuição tem seu próprio vetor média, o qual é denominado segundo momento trigonométrico, ou seja, o segundo momento trigonométrico amostral é o vetor média amostral das observações duplicadas.

Em geral, podemos definir o p-ésimo momento trigonométrico amostral em torno da direção zero como:

$$
\mathrm{m}_{\mathrm{p}}^{\prime}=\left(\bar{C}_{p}, \bar{S}_{p}\right),
$$

onde

$$
\begin{aligned}
\bar{C}_{p} & =\frac{1}{n} \sum_{i=1}^{n} \cos p \theta_{i} e \\
\bar{S}_{p} & =\frac{1}{n} \sum_{i=1}^{n} \operatorname{sen} p \theta_{i},
\end{aligned}
$$


podendo tambem ser escrito como:

$$
\mathrm{m}_{\mathrm{p}}^{\prime}=\bar{C}_{p}+i \bar{S}_{p}=r_{p} e^{i \bar{\theta}_{p}},
$$

onde

$$
\begin{aligned}
& r_{p}=\left(\bar{C}_{p}{ }^{2}+\bar{S}_{p}{ }^{2}\right)^{1 / 2} e \\
& \overline{\theta_{p}}=\text { direção do p-ésimo momento amostral. }
\end{aligned}
$$

O p-ésimo momento trigonométrico amostral centrado na direção média é definido como:

$$
\mathrm{m}_{\mathrm{p}}=\left(\bar{C}_{p}^{*}, \bar{S}_{p}^{*}\right)
$$

onde:

$$
\begin{aligned}
\bar{C}_{p}{ }^{*} & =\frac{1}{n} \sum_{i=1}^{n} \cos p\left(\theta_{i}-\bar{\theta}\right) \mathrm{e} \\
\bar{S}_{p}{ }^{*} & =\frac{1}{n} \sum_{i=1}^{n} \operatorname{senp}\left(\theta_{i}-\bar{\theta}\right),
\end{aligned}
$$

podendo tambem ser escrito como:

$$
\mathbf{m}_{\mathbf{p}}=\bar{C}_{p}^{*}+i \bar{S}_{p}^{*}
$$

Em particular ,

$$
\begin{aligned}
\mathbf{m}_{1} & =\frac{1}{n} \sum_{i=1}^{n} \cos \left(\theta_{i}-\bar{\theta}\right)+i \frac{1}{n} \sum_{i=1}^{n} \operatorname{sen}\left(\theta_{i}-\bar{\theta}\right)=\frac{1}{n} \sum_{i=1}^{n} \cos \left(\theta_{i}-\bar{\theta}\right) \quad e \\
\mathbf{m}_{\mathbf{2}} & =\frac{1}{n} \sum_{i=1}^{n} \cos 2\left(\theta_{i}-\bar{\theta}\right)+i \frac{1}{n} \sum_{i=1}^{n} \operatorname{sen} 2\left(\theta_{i}-\bar{\theta}\right)
\end{aligned}
$$

\subsection{Dispersão circular amostral e erro padrão cir- cular}

Considerando o segundo momento trigonométrico centrado na média, é possível definir uma outra medida de dispersão denominada dispersão circular amostral, dada por: 


$$
\hat{\delta}=\frac{1-r_{2}}{2 r^{2}}
$$

onde

$$
\begin{aligned}
r & =\frac{1}{n} \sum_{i=1}^{n} \cos \left(\theta_{i}-\bar{\theta}\right) e \\
r_{2} & =\left[\left(\frac{1}{n} \sum_{i=1}^{n} \cos 2\left(\theta_{i}-\bar{\theta}\right)\right)^{2}+\left(\frac{1}{n} \sum_{i=1}^{n} \operatorname{sen} 2\left(\theta_{i}-\bar{\theta}\right)\right)^{2}\right]^{1 / 2}
\end{aligned}
$$

Conforme veremos posteriormente a dispersão circular amostral é muito utilizada em inferências sobre a direção média.

A partir da dispersão circular amostral, definimos o erro padrão circular como:

$$
\hat{\sigma}=\left(\frac{\hat{\delta}}{n}\right)^{1 / 2}
$$

Exemplo 2.6.1 Considerando os dados do Exemplo 2.2.1, obtemos que o comprimento do vetor do primeiro e segundo momentos trigonométricos amostrais são, respectivamente, $r=0,7109$ e $r_{2}=0,5955$. Assim a dispersão circular amostral é $\hat{\delta}=0,1023$ e o erro padrão circular é $\hat{\sigma}=(0,1023 / 9)^{1 / 2}=0,107$.

\subsection{Medidas de curtose e assimetria}

Medidas de assimetria e curtose em uma amostra de dados circulares são, respectivamente, definidas como:

$$
\begin{aligned}
\hat{S} & =\frac{r_{2} \operatorname{sen}\left(\overline{\theta_{2}}-2 \bar{\theta}\right)}{(1-r)^{3 / 2}} e \\
\hat{K} & =\frac{r_{2} \cos \left(\overline{\theta_{2}}-2 \bar{\theta}\right)-(1-V)^{4}}{(1-r)^{2}}
\end{aligned}
$$

onde

$$
\begin{aligned}
r & : \text { é o comprimento do vetor média amostral } \\
V & =1-r \text { e } \\
\bar{\theta}_{2} & : \text { é a direção do segundo momento trigonométrico amostral. }
\end{aligned}
$$


Distribuições de frequência simétricas tenderão a ter valores de $\hat{S}$ próximos de zero. Distribuições com o mesmo grau de achatamento da normal terão $\hat{K}$ aproximadamente igual zero.

Exemplo 2.7.1 Tendo em conta os dados do Exemplo 2.2.1, obtemos $\bar{\theta}_{2}=2,287$; $\hat{S}=1,75$ e $\hat{K}=3,3836$.

\subsection{Modificações para dados axiais}

As técnicas usadas para análise de dados vetoriais também podem ser utilizadas para dados axiais após uma modificação nos dados.

Quando temos dados axiais, as obsevações são restritas a assumir valores em um arco de comprimento $180^{\circ}$ (isto é, podemos reduzir todos os ângulos módulo $180^{\circ}$ ). Para poder calcular as medidas de locação e dispersão apresentadas anteriormente, temos que eliminar esta restrição, isto é, temos que relacionar o período de $180^{\circ} \mathrm{com}$ $360^{\circ}$, da mesma forma que relacionamos $24 h$ com $360^{\circ}$ em medidas de tempo. Fisher e Powell(1989) e Fisher(1993) sugerem que, na análise de dados axiais seja utilizado o seguinte procedimento:

1. Duplica-se todos os valores das observações, módulo $360^{\circ}$. Ao duplicarmos os ângulos dizemos que estamos transformado os dados axiais em vetoriais.

2. Faz-se a análise desejada, considerando dados transformados.

3. No cálculo de medidas de posição, cancela-se o efeito da duplicação dividindo por 2 o resultado obtido. Recomenda-se que as medidas de dispersão sejam deixadas em unidades vetoriais. 


\section{Capítulo 3}

\section{Modelos}

\subsection{Introdução}

No capítulo 2, definimos algumas estatísticas apropriadas à descrição de uma amostra de dados circulares. Em algumas situações, o conhecimento do mecanismo gerador das observações nos permite supor um modelo probabilístico que caracterize o comportamento dos dados. Ajustando o modelo segundo algum critério apropriado, podemos resumir eficientemente nossos dados através das estimativas dos parâmetros do modelo probabilístico especificado.

A maioria dos modelos circulares são análogos circulares de distribuições univariadas importantes.

Descreveremos neste trabalho alguns modelos de probabilidade contínuos utilizados com maior frequência na análise de dados circulares: as distribuições circular uniforme, a normal enrolada e, mais detalhadamente, a de von Mises, por ser a considerada com maior frequência na análise de dados circulares. Outros modelos discretos e contínuos podem ser encontrados em Mardia (1972).

Denotaremos por $f(\theta)$ a função de densidade de probabilidade da variável aleatória circular contínua $\Theta$, isto é, uma função contínua tal que:

i) $f(\theta) \geq 0, \quad-\infty<\theta<\infty$;

ii) $f(\theta+2 \pi)=f(\theta), \quad-\infty<\theta<\infty$;

iii) $\int_{0}^{2 \pi} f(\theta) d \theta=1$. 
A função de distribuição $F(\theta)$ correspondente a $f(\theta)$ pode ser definida sobre qualquer intervalo $\left(\theta_{1}, \theta_{2}\right)$ como:

$$
F\left(\theta_{2}\right)-F\left(\theta_{1}\right)=\int_{\theta_{1}}^{\theta_{2}} f(\theta) d \theta .
$$

Entretanto, é conveniente definirmos um ponto inicial para a distribuição, como por exemplo $0^{\circ}$, e assim:

$$
F(\theta)=\int_{0}^{\theta} f(\phi) d \phi
$$

Em particular, $F(2 \pi)=1$

\subsection{Momentos de distribuições circulares}

Distribuições na reta são usualmente descritas por seus momentos. Conforme vimos na seção 2.5, quando apresentamos os momentos trigonométricos amostrais, as definições de momentos utilizados na reta não se aplicam ao caso circular. Vamos definir nesta seção, os momentos trigonométricos populacionais que definem de forma única as distribuições circulares. $\mathrm{O}$ momento trigonométrico de ordem $p$ é definido por:

$$
\boldsymbol{\mu}_{p}^{\prime}=\left[\begin{array}{l}
\alpha_{p}^{\prime} \\
\beta_{p}^{\prime}
\end{array}\right]=\left[\begin{array}{c}
E(\cos p \theta) \\
E(\operatorname{sen} p \theta)
\end{array}\right]
$$

O par $\left(\alpha_{p}^{\prime}, \beta_{p}^{\prime}\right)$ pode ser representado graficamente, como na Figura 3.1 denotemos por $\rho_{p}$ o comprimento do vetor $\mu_{p}^{\prime}$. Considerando suas coordenadas polares do par $\left(\alpha_{p}^{\prime}, \beta_{p}^{\prime}\right)$ temos que:

$$
\boldsymbol{\mu}_{p}^{\prime}=\left[\begin{array}{c}
\alpha_{p}^{\prime} \\
\beta_{p}^{\prime}
\end{array}\right]=\left[\begin{array}{c}
\rho_{p} \cos \mu_{p}^{\prime} \\
\rho_{p} \operatorname{sen} \mu_{p}^{\prime}
\end{array}\right] .
$$

Então em notação complexa :

$$
\begin{aligned}
\boldsymbol{\mu}_{p}^{\prime} & =\alpha_{p}^{\prime}+i \beta_{p}^{\prime} \\
& =\rho_{p} \cos \mu_{p}^{\prime}+i \rho_{p} \operatorname{sen} \mu_{p}^{\prime} \\
& =\rho_{p} e^{i \mu_{p}^{\prime}} .
\end{aligned}
$$


Para $p=1$, temos o momento trigonométrico de primeira ordem:

$$
\boldsymbol{\mu}_{1}^{\prime}=\left[\begin{array}{l}
\alpha_{1}^{\prime} \\
\beta_{1}^{\prime}
\end{array}\right]=\left[\begin{array}{c}
E(\cos \theta) \\
E(\operatorname{sen} \theta)
\end{array}\right] .
$$

Neste caso, $\alpha_{1}^{\prime}=\rho \cos \mu, \beta_{1}^{\prime}=\rho \operatorname{sen} \mu$, onde $\mu$ é a direção média, $\rho=\rho_{1}$ é o comprimento do vetor média e $\boldsymbol{\mu}_{1}^{\prime}$ é o vetor de média.

O $p$ - ésimo momento trigonométrico centrado na direção média $\mu$ é definido da seguinte maneira:

$$
\boldsymbol{\mu}_{p}=\left[\begin{array}{l}
\alpha_{p} \\
\beta_{p}
\end{array}\right]=\left[\begin{array}{l}
E(\cos p(\theta-\mu)) \\
E(\operatorname{sen} p(\theta-\mu))
\end{array}\right]
$$

Considerando as coordenadas polares correspondentes a $\left(\alpha_{p}, \beta_{p}\right)$ :

$$
\boldsymbol{\mu}_{p}=\left[\begin{array}{c}
\alpha_{p} \\
\beta_{p}
\end{array}\right]=\left[\begin{array}{c}
\rho_{p} \cos \mu_{p} \\
\rho_{p} \operatorname{sen} \mu_{p}
\end{array}\right]
$$

ou, utilizando a notação de números complexos

$$
\boldsymbol{\mu}_{p}=\alpha_{p}+i \beta_{p}=\int_{0}^{2 \pi} \cos p(\theta-\mu) f(\theta) d \theta+i \int_{0}^{2 \pi} \operatorname{senp}(\theta-\mu) f(\theta) d \theta=\rho_{p} e^{i \mu_{p}}
$$

Para $p=1$, se obtém $\alpha_{1}=\rho$ e $\beta_{1}=0$, pois

$$
\int_{0}^{2 \pi} \cos \theta d \theta=\rho \cos \mu \quad e \quad \int_{0}^{2 \pi} \operatorname{sen} \theta d \theta=\rho \operatorname{sen} \mu
$$

de modo que :

$$
\begin{aligned}
\int_{0}^{2 \pi} \cos (\theta-\mu) d \theta & =\int_{0}^{2 \pi}(\cos \theta \cos \mu+\operatorname{sen} \theta \operatorname{sen} \mu) d \theta \\
& =\cos \mu \int_{0}^{2 \pi} \cos \theta d \theta+\operatorname{sen} \mu \int_{0}^{2 \pi} \operatorname{sen} \theta d \theta \\
& =\rho\left(\cos ^{2} \mu+\operatorname{sen}^{2} \mu\right) \\
& =\rho
\end{aligned}
$$

e

$$
\begin{aligned}
\int_{0}^{2 \pi} \operatorname{sen}(\theta-\mu) d \theta & =\int_{0}^{2 \pi}(\operatorname{sen} \theta \cos \mu-\cos \theta \operatorname{sen} \mu) d \theta \\
& =\cos \mu \int_{0}^{2 \pi} \operatorname{sen} \theta d \theta-\operatorname{sen} \mu \int_{0}^{2 \pi} \cos \theta d \theta \\
& =\rho(\cos \mu \operatorname{sen} \mu-\operatorname{sen} \mu \cos \mu) \\
& =0
\end{aligned}
$$




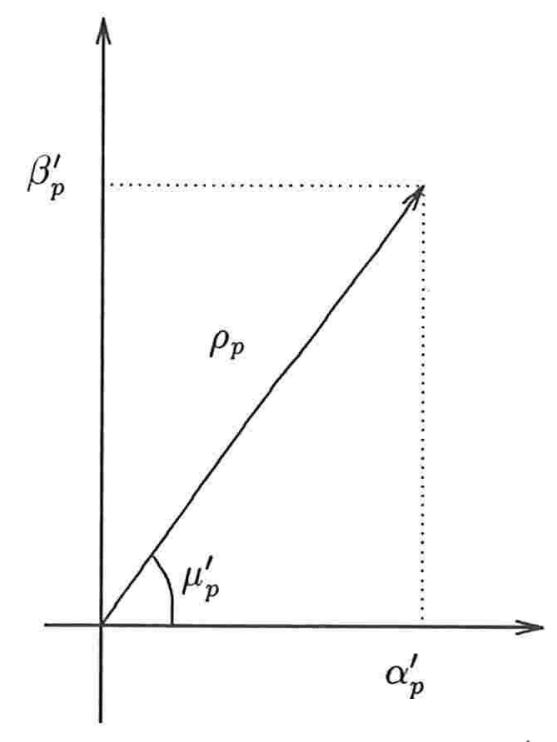

Figura 3.1: Representação gráfica do momento trigonométrico de ordem p.

As estatísticas correspondentes aos parâmetros populacionais $\boldsymbol{\mu}_{p}^{\prime}, \boldsymbol{\mu}_{p}, \rho_{p}, \mu, \mu_{p}, \alpha_{p}^{\prime}$ e $\beta_{p}^{\prime}$ são dadas respectivamente por: $\mathbf{m}_{p}^{\prime}, \mathbf{m}_{p}, r_{p}, \bar{\theta}, \bar{\theta}_{p}, \bar{C}_{p}$ e $\bar{S}_{p}$ definidas no capítulo 2 .

Definimos a seguir alguns parâmetros de grande interesse no estudo de distribuições circulares e que são funções dos primeiro e segundo momentos trigonométricos:

$$
\begin{array}{ll}
\text { variância circular de } \Theta & : \vee=1-\rho, \quad 0 \leq \vee \leq 1 . \\
\text { desvio padrão circular } & : \sigma=(-2 \log \rho)^{1 / 2} . \\
\text { dispersão circular } & : \delta=\frac{\left(1-\rho_{2}\right)}{2 \rho^{2}} . \\
\text { coeficiente de curtose } & : \mathrm{K}=\frac{\left(\alpha_{2}-\rho^{4}\right)}{(1-\rho)^{2}} . \\
\text { coeficiente de assimetria } & : \mathrm{S}=\frac{\beta_{2}}{(1-\rho)^{3 / 2}} .
\end{array}
$$

As estatísticas correspondentes a esses parâmetros foram definidas no capítulo 2 . 


\subsection{Propriedades do vetor média e da variância circular}

1. $E(\operatorname{sen}(\theta-\mu))=0$, onde $\mu$ é a direção média de $\Theta$.

2. $V=1-\rho, \quad 0 \leq V \leq 1$.

3. Se $V=0$ a distribuição é concentrada em um único ponto $\Theta=\mu$. Se $V=1$, a distribuição tem máxima variância e não existe concentração em torno de qualquer direção.

4. A variância circular em torno do ângulo $\theta=\nu$ é dada por $V^{\prime}=1-E(\cos (\theta-\nu))$. As quatidades $V^{\prime}$ e $V$ são relacionados por $V^{\prime}=V+2 \rho \operatorname{sen}^{2} \frac{1}{2}(\mu-\nu)$. Além disso, $V^{\prime}$ assume valor mínimo quando $\nu=\mu$.

5. $V$ é invariante sob qualquer mudança da direção zero. Assim se fizermos a transformação: $\Theta^{*}=(\Theta-\nu) \quad(\bmod 2 \pi)$, temos que $\mu^{*}=(\mu-\nu) \quad(\bmod 2 \pi)$, e $V^{*}=$ $V$.

\subsection{Distribuições circulares}

Nesta seção apresentaremos algumas distribuições circulares e suas propriedades mais importantes. Outras distribuições podem ser encontradas, por exemplo, em Fisher(1993).

\subsubsection{Distribuição uniforme}

Nesse modelo todas as direções entre 0 e $2 \pi$ são escolhidas com igual probabilidade, ou seja, não existe direção preferencial.

A densidade de probabildade é constante sobre a circunferência de raio unitário, isto é :

$$
f(\theta)=\frac{1}{2 \pi}, \quad 0 \leq \theta \leq 2 \pi
$$


(ver Figura 3.2 ). Sua função de distribuição é dada por

$$
F(\theta)=\frac{\theta}{2 \pi}, \quad 0 \leq \theta \leq 2 \pi .
$$

A direção média $\mu$ não é definida. O comprimento médio da resultante $\rho$ é igual a zero. Consequentemente temos que :

$$
\begin{gathered}
V=1 \\
\delta=\infty \quad e \\
\mu_{p}=\left[\begin{array}{l}
\alpha_{p} \\
\beta_{p}
\end{array}\right]=\left[\begin{array}{l}
0 \\
0
\end{array}\right] \text { para } p \geq 1 .
\end{gathered}
$$

Figura 3.2: Representação gráfica da distribuição uniforme circular.

\subsubsection{Distribuição normal enrolada}

É uma distribuição unimodal e simétrica obtida envolvendo-se o círculo de raio unitário por uma distribuição normal . Essa função densidade é construída da seguinte forma: seja $X$ uma variável aleatória na reta com função densidade $g(x)$ dada por uma $N\left(\mu, \sigma^{2}\right)$. Podemos definir uma variável circular $\Theta$ como:

$$
\Theta=X \quad(\bmod 2 \pi)
$$


A função densidade de probabilidade $f(\theta)$ de $\Theta$ é dada por:

$$
\begin{aligned}
f(\theta) & =\sum_{k=-\infty}^{\infty} g(\theta+2 k \pi) \\
& =\frac{1}{\sigma \sqrt{2 \pi}} \sum_{k=-\infty}^{\infty} \exp \left(-\frac{1}{2} \frac{((\theta-\mu)+2 k \pi)^{2}}{\sigma^{2}}\right), \quad 0<\theta \leq 2 \pi .
\end{aligned}
$$

Mardia(1972) mostrou que a expressão acima pode ser escrita como:

$$
f(\theta)=\frac{1}{2 \pi}\left[1+2 \sum_{p=1}^{\infty} \rho^{p^{2}} \cos p(\theta-\mu)\right], \quad 0 \leq \theta \leq 2 \pi, \quad 0 \leq \rho \leq 1,
$$

onde $\mu$ é a direção média e $\rho$ é o comprimento do vetor média. Nesta distribuição temos que dispersão circular $\delta$ é dada por:

$$
\delta=\frac{\left(1-\rho^{4}\right)}{\left(2 \rho^{2}\right)}
$$

e o p-ésimo momento trigonométrico central é dado por:

$$
\boldsymbol{\mu}_{p}=\left[\begin{array}{c}
\alpha_{p} \\
\beta_{p}
\end{array}\right]=\left[\begin{array}{c}
\rho^{p^{2}} \\
0
\end{array}\right], \quad p \geq 1
$$

onde

$$
\rho=e^{\left(-\sigma^{2} / 2\right)}, \quad \text { ou } \quad \sigma^{2}=-2 \log \rho
$$

Quando $\rho \rightarrow 0$, a distribuição converge à distribuição uniforme; quando $\rho \rightarrow$ 1 a distribuição converge à distribuição degenerada na direção média $\mu$, isto é, a distribuição é concentrada em um único ponto $\theta=\mu$.

\subsubsection{Distribuição de von Mises}

Uma variável aleatória circular $\Theta$ tem distribuição de von Mises, $V M(\mu, \kappa)$, se sua função de densidade de probabilidade é dada por:

$$
f(\theta)=\left[2 \pi I_{0}(\kappa)\right]^{-1} \exp (\kappa \cos (\theta-\mu)), \quad 0 \leq \theta \leq 2 \pi, \quad 0 \leq \kappa<\infty,
$$

onde

$\mu$ é o parâmetro que representa a direção média,

$\kappa$ é um parâmetro associado á concentração e 


$$
I_{0}(\kappa)=\sum_{r=0}^{\infty}(r !)^{-2}\left(\frac{1}{2} \kappa\right)^{2 r}
$$

é a função Bessel modificada de ordem zero (ver, por exemplo, Mardia(1972), Apêndice 1).

Esta distribuição foi introduzida por von Mises em 1918 para estudar desvios de medidas de pesos atômicos. Apesar de não haver relação entre essa aplicação e dados circulares, a distribuição de von Mises é atualmente a base da inferência estatística paramétrica para esse tipo de dados.

Esta distribuição é considerada o análogo natural no círculo da distribuição normal na reta. Na prática, ela é um modelo razoável para conjuntos de dados circulares unimodais simétricos que ocorrem em diferentes áreas de aplicação.

Como $\cos (x)=\cos (-x)$, a função de densidade (3.21) é simétrica em torno da direção média $\mu$. O valor mínimo do parâmetro de concentração $\kappa$ é zero e, neste caso, $f(\theta)=\frac{1}{2 \pi}$, ou seja, quando a concentração é minima, a distribuição de von Mises coincide com a distribuição circular uniforme. A moda da distribuição é $\theta=\mu$ e a antimoda é $\theta=\mu+\pi$ (ver Figura 3.3).

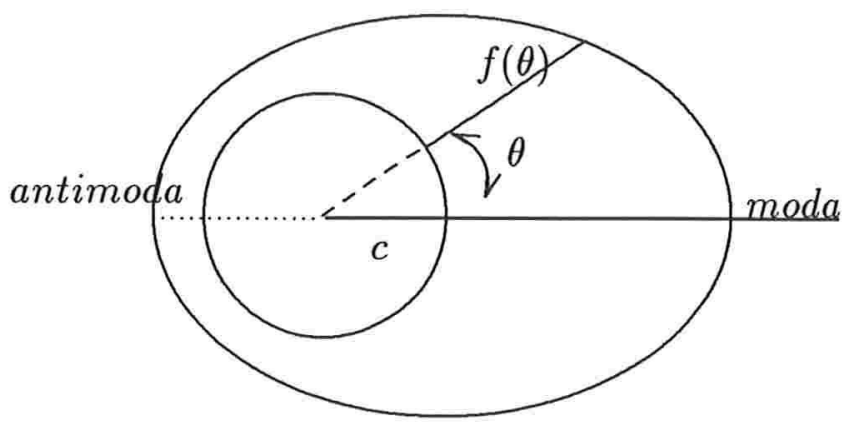

Figura 3.3: Representação polar da distribuição de von Mises $(\mu, \kappa)$.

A razão entre os valores da densidade na moda e na antimoda é $e^{2 \kappa}$, e assim, quanto maior o valor de $\kappa$, maior é a concentração em torno da direção modal.

Na Figura 3.4 representamos a funcão da densidade de von Mises com $\mu=0 \mathrm{e}$ diferentes valores de $\kappa$. Na Figura 3.5 representamos a densidade de von Mises para $\kappa=1$ e diferentes valores de $\mu$. 


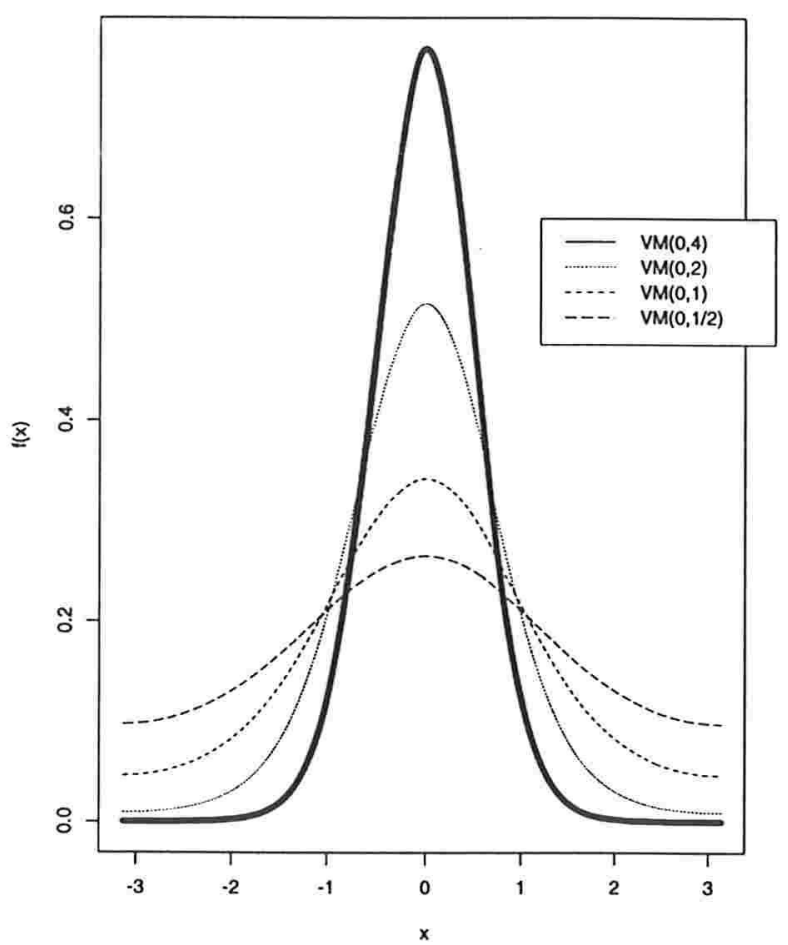

Figura 3.4: Representação da distribuição de von Mises para: $\mu_{0}=0$ e $\kappa=$ $1 / 2,1,2,4$.

O p-ésimo momento central é dado por:

$$
\boldsymbol{\mu}_{p}=\left[\begin{array}{c}
\alpha_{p} \\
\beta_{p}
\end{array}\right]=\left[\begin{array}{c}
\rho_{p} \\
0
\end{array}\right]
$$

onde

$\rho_{p}$ é o comprimento do vetor $\boldsymbol{\mu}_{p}$ e pode ser escrito como:

$$
\begin{aligned}
\rho_{p} & =I_{p}(\kappa) / I_{0}(\kappa) \\
I_{p}(\kappa) & =\sum_{r=0}^{\infty}((r+p) ! r !)^{-1}\left(\frac{1}{2} \kappa\right)^{2 r+p}, \quad p=1,2, \ldots
\end{aligned}
$$

é função Bessel modificada de primera classe e de p-ésima ordem e $I_{0}(\kappa)$ é como na expressão (3.22). 


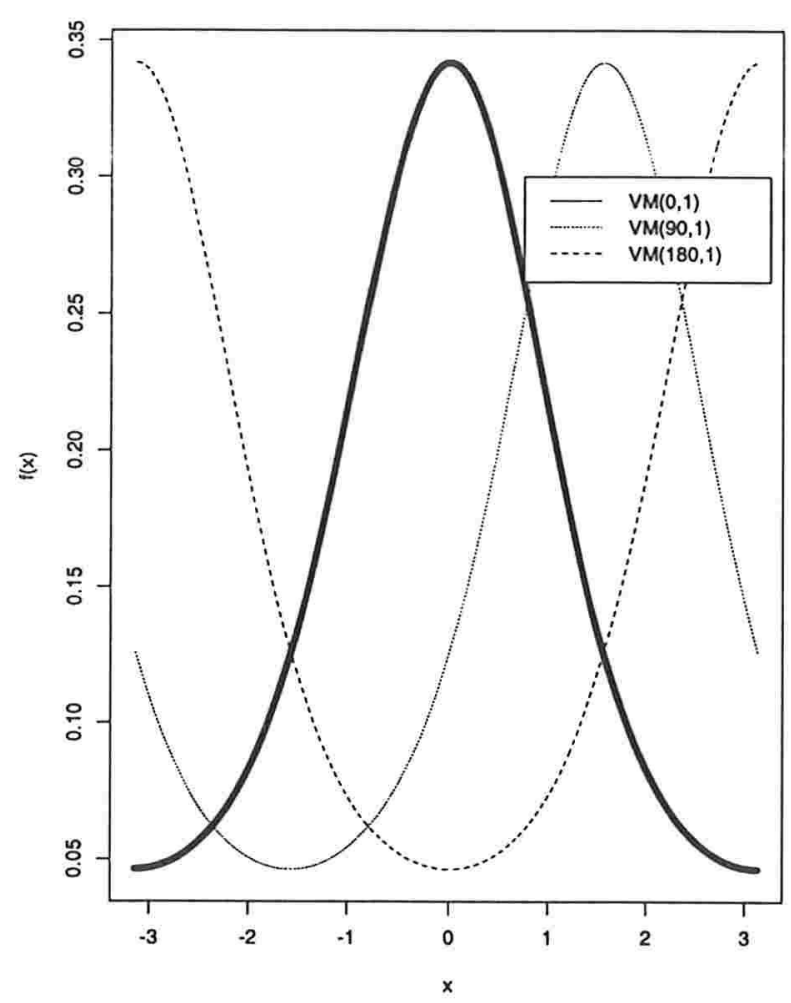

Figura 3.5: Representação da densidade de distribuição de von Mises para: $\mu=$ $0, \pi / 2, \pi$, e $\kappa=1$.

O primeiro momento da distribuição, quando $\mu \neq 0$, é dado por:

$$
\boldsymbol{\mu}=\left[\begin{array}{l}
\alpha \\
\beta
\end{array}\right]=\left[\begin{array}{l}
\rho \cos \mu \\
\rho \operatorname{sen} \mu
\end{array}\right]
$$

onde

$\rho$ é o comprimento do vetor média e pode ser escrito como:

$$
\rho=I_{1}(\kappa) / I_{0}(\kappa)=A(\kappa) \text {. }
$$

A função de distribuição é dada por:

$$
F(\theta)=\left[2 \pi I_{0}(\kappa)\right]^{-1} \int_{0}^{\theta} \exp (\kappa \cos (\phi-\mu) d \phi
$$


Batschelet(1981) e Mardia(1972) fornecem tabelas da função de distribuição para valores de $\kappa$ no intervalo $[0,10]$.

\section{Algumas propriedades e notações}

1. Uma propriedade importante da distribuição normal $N\left(\mu, \sigma^{2}\right)$ na reta é que o intervalo $\mu \pm k \sigma$, para algum $k>0$, é um intervalo com probabilidade fixa (aproximadamente 0.683 para $\mu \pm \sigma$ )indepedente do valor de $\sigma>0$. A razão para isto é que a variavél aleatória $X \sim N\left(\mu, \sigma^{2}\right)$ pode ser transformada em uma variavél aleatória padronizada $Z \sim N(0,1)$ através de uma simples mudança de locação e escala: $Z=\left(\frac{X-\mu}{\sigma}\right)$. Isto não ocorre com uma variável aleatória que se distribui como uma von Mises $V M(\mu, \kappa)$, que pode ser centrada em $\mu$ mas não pode ser reescalonada de forma a ter concentração igual à unidade. Este fato dificulta a utilização da distribuição de von Mises na prática, pois não é possível a construção de tabelas com uma distribuição padrão.

2. Outra dificuldade na utilização da distribuição de von Mises é causada pelo difícil cálculo das funções de Bessel modificadas $I_{0}(\kappa), I_{1}(\kappa), \ldots$. Apresentamos a seguir um procedimento recursivo para o cálculo dessas expressões. Outras referências sobre o cálculo e propriedades dessas funções podem ser encontradas em Mardia(1972), Baschelet(1981), Watson(1983) e Abramowitz e Stegum(1970). Temos que:

$$
I_{0}(\kappa)=\sum_{r=0}^{\infty}(r !)^{-2}\left(\frac{1}{2} \kappa\right)^{2 r} .
$$

No geral,

$$
\begin{aligned}
& I_{p}(\kappa)=\sum_{r=0}^{\infty}((r+p) ! r !)^{-1}\left(\frac{1}{2} \kappa\right)^{2 r+p}, \quad p=1,2, \ldots \\
& A_{p}(\kappa)=\frac{I_{p}(\kappa)}{I_{0}(\kappa)}, \quad p=0,1,2, \ldots
\end{aligned}
$$

Para calcular valores de $I_{p}(\kappa)$ e $A_{p}(\kappa)$ para $p \geq 2$, basta avaliar estas funções para $p=0$ e $p=1$, pois as seguintes relações de recorrência podem ser usadas para $p=2,3, \ldots$

$$
\begin{aligned}
& I_{p}(\kappa)=I_{p-2}(\kappa)-\frac{2(p-1)}{\kappa} I_{p-1}(\kappa) e \\
& A_{p}(\kappa)=A_{p-2}(\kappa)-\frac{2(p-1)}{\kappa} A_{p-1}(\kappa) .
\end{aligned}
$$


Em particular,

$$
\begin{aligned}
I_{2}(\kappa) & =I_{0}(\kappa)-\frac{2}{\kappa} I_{1}(\kappa) e \\
A_{2}(\kappa) & =1-\frac{2}{\kappa} A_{1}(\kappa) .
\end{aligned}
$$

Aproximações para $I_{0}(\kappa)$ e $I_{1}(\kappa)$ são dadas em Abramowitz e Stegun (1970). Nas Figuras 3.6 apresentamos gráficos de $I_{0}(\kappa), I_{1}(\kappa)$, e $A_{1}(\kappa)$. Observamos que essas funções são monotônicas crescentes.

\section{Relações com outras distribuições}

Apresentamos a seguir relações existentes entre a distribuição von Mises e outras distribuições circulares.

\section{von Mises e uniforme}

Quando fazemos $\kappa=0$ em (3.21) observamos que a distribuição von Mises se reduz a uma distribuição uniforme circular.

\section{von Mises e normal enrolada}

A distribuição normal enrolada tem forma bastante similar à da distribuição de von Mises. Observando as expressões do comprimento do vetor média $\rho$ nas duas distribuições, dadas nas expressões (3.20) e (3.26) observamos que, quando as dispersões aumentam, ambas distribuições convergem para a uniforme circular. Quando $\kappa \rightarrow \infty$ e $\sigma \rightarrow 0$ e as duas distribuições convergem para uma distribuição degenerada no mesmo ponto. Portanto, para casos extremos existe concordância entre as distribuições. Stephens(1963) verificou que existe concordância satisfatória também para valores intermediários.

Observemos que, para grandes valores de $\kappa$, a distribuição von Mises se aproxima da normal.

No Apêndice C apresentamos um programa computacional para gerar dados com distribuição de von Mises baseados no algoritmo proposto por Best e Fisher(1979). 

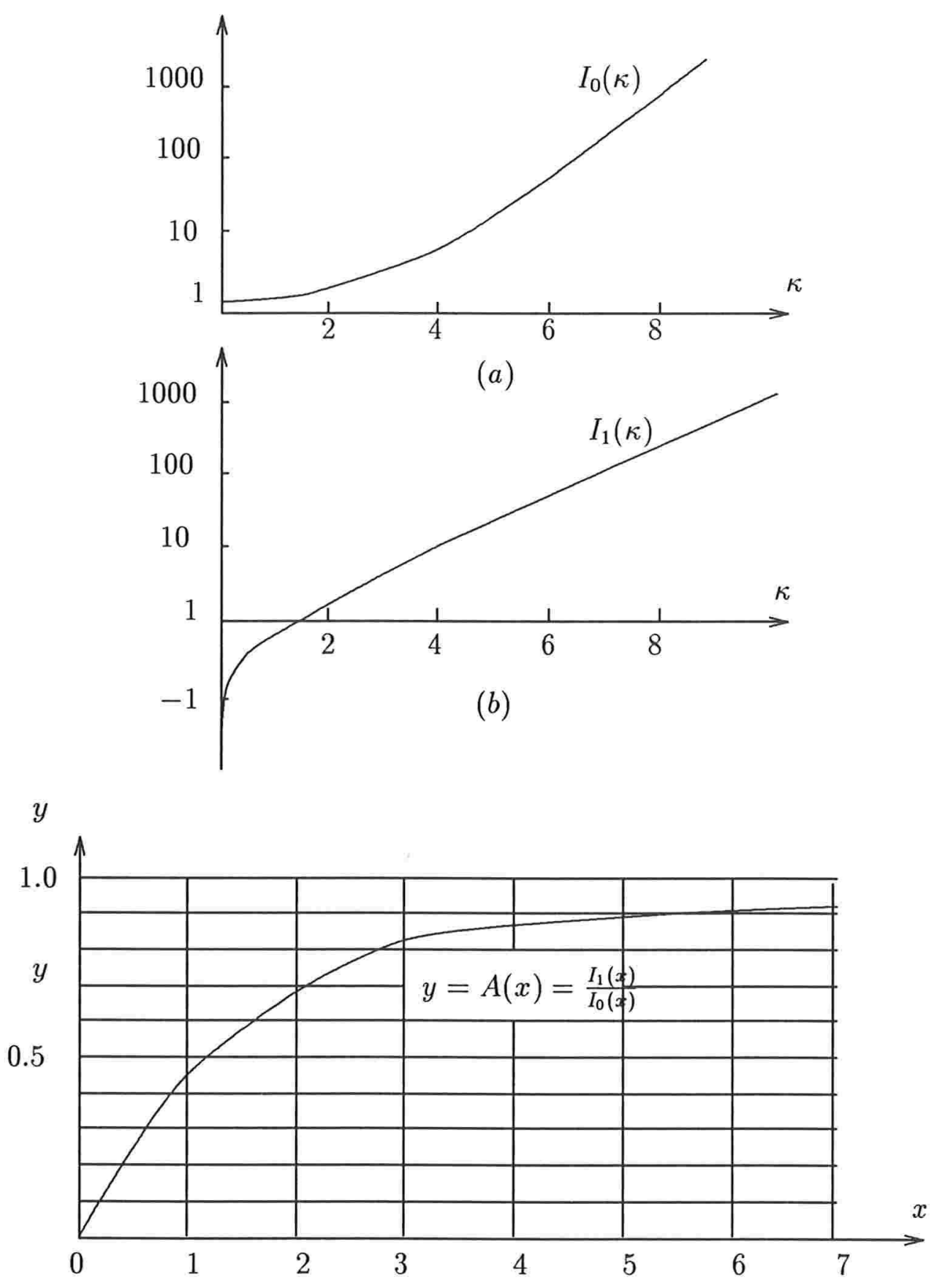

(c)

Figura 3.6: Gráficos das funções Bessel $(a) I_{0}(\kappa),(b) I_{1}(\kappa)$ e $(c) A_{1}(\kappa)$. 


\section{Capítulo 4}

\section{Análise de uma amostra}

\subsection{Introdução}

Neste capítulo os métodos que apresentaremos estão relacionados com a análise de uma amostra de observações independentes, $\theta_{1}, \ldots, \theta_{n}$, de uma população circular.

Como um primeiro passo da análise, sugerimos que as técnicas apresentadas no capítulo 2 sejam aplicadas, para termos indicação sobre o comportamento dos dados. A construção de gráficos do tipo dos apresentados na Seção 2.2 podem sugerir, embora de forma grosseira, se os dados provêm de uma distribuição uniforme, unimodal ou multimodal e indicar possíveis "outliers". Se os dados corresponderem a uma amostra de uma distribuição uniforme circular, não faz sentido prosseguirmos a análise buscando estabelecer uma direção de preferência. Nos casos em que esses gráficos indicam que a população é unimodal, o cálculo das medidas de posição e dispersão dadas nas Seções 2.3 e 2.4 fornecem um resumo dos dados.

$\mathrm{Na}$ Seção 4.2 apresentamos métodos para a verificação da uniformidade que devem ser aplicados após a análise descritiva. Para dados unimodais, apresentamos na Seção 4.3 métodos não paramétricos para a estimação e testes de hipótese sobre medidas de locação. Na Seção 4.4 descrevemos técnicas de estimação e testes de hipótese sobre os parâmetros de posição e concentração de uma distribuição de von Mises. 


\subsection{Verifição de uniformidade}

Nesta seção apresentamos dois métodos para verificação de uniformidade de uma amostra de medidas circulares: um método gráfico e outro inferencial.

\subsubsection{Método gráfico}

Antes de proceder a um teste formal de uniformidade, é adequado construirmos um gráfico de probabilidades uniforme. Para isto, obtemos as estatísticas de ordem como na reta $\theta_{(1)} \leq \ldots \leq \theta_{(n)}$ da amostra como foi descrito na Subseção 2.4.5, e calculamos $x_{1}=\frac{\theta_{(1)}}{2 \pi}, \ldots, x_{n}=\frac{\theta_{(n)}}{2 \pi}$. Construimos, então, um gráfico dos pontos

$$
\left(\frac{1}{n+1}, x_{1}\right), \ldots,\left(\frac{n}{n+1}, x_{n}\right) \text {. }
$$

Se o modelo é uniforme, os pontos devem se localizar aproximadamente ao longo da reta de $45^{0}$ que passa pelo ponto $(0,0)$.

\subsubsection{Teste de Rayleigh}

Existe na literatura de dados circulares mais de um teste para a verificação da uniformidade (ver, por exemplo, Fisher,(1993)). A escolha entre eles depende do modelo especificado na hipótese alternativa. Por exemplo, se estivermos interessados em detectar qualquer forma de não-uniformidade, o teste geral 'ommnibus test' descrito em Fisher(1993) é adequado. Como, em geral, temos interesse em verificar se os dados correspondem a uma amostra de uma população unimodal, vamos considerar o teste de Rayleigh, descrito em Batschelet(1981), para testar as hipóteses:

$$
\begin{aligned}
& H_{0}: A \text { distribuição é uniforme } \\
& H_{1}: \text { A distribuição é unimodal. }
\end{aligned}
$$

Este procedimento se baseia no fato de que o comprimento do vetor média amostral, $r$, dá uma ideia de concentração em torno de uma única direção. Para amostras 
da distribuição uniforme circular, o valor de $r$, em geral, é pequeno. Como, sob a hipótese de que os dados seguem uma distribuição uniforme, a distribuição amostral de $r$ é conhecida (ver, Mardia(1972) seção 4.4), então $r$ é uma estatística natural para o teste. A hipótese de uniformidade é rejeitada para valores grandes de $r$.

Batschelet(1981) e Mardia(1972) fornecem os níveis descritivos associados a valores observados da estatística do teste para diferentes tamanhos da amostra. Valores críticos para o teste também podem ser encontrados em Zar(1974). Quando o tamanho da amostra é muito grande a estatística

$$
Z=n r^{2}
$$

pode ser usada para testar a hipótese (4.1). Neste caso rejeita-se $H_{0}$ se o valor observado de $Z$ é grande. Os valores críticos para o teste podem ser encontrados em Batschelet(1981) ou Zar(1974).

\subsection{Métodos não paramétricos para a análise de dados unimodais}

Nesta seção estudaremos técnicas de inferência não paramétrica para dados unimodais. Nosso problema de interesse é estimar a direção de preferência, e testar se essa direção concorda com algum valor especificado. Como direção preferida consideramos a direção mediana e a direção média.

\subsubsection{Estimação da direção mediana}

Consideramos a estimação da direção mediana $\tilde{\mu}$ em uma amostra por ponto e por intervalo. O estimador pontual $\tilde{\theta}$ foi apresentado na Seção 2.3. Para a estimação de $\tilde{\mu}$ por intervalo, vamos assumir que a dispersão da amostra seja pequena, isto é, os dados estão concentrados em um arco de comprimento substancialmente menor que a circunferência toda. Desta forma podemos construir intervalos de confiança para $\tilde{\mu}$ adotando os procedimentos utilizados em dados na reta, descritos, por exemplo, em Conover(1971). Lembremos que a construção desses intervalos está relacionada com a região de aceitação do teste de hipóteses: 


$$
\begin{array}{ll}
H_{0}: & p=1 / 2 \\
H_{1}: & p \neq 1 / 2
\end{array}
$$

em uma distribuição $B(n, p)$. Consideremos a amostra ordenada $\theta_{(1)}, \ldots, \theta_{(n)}$.

Para $n<16$, Fisher e Powell(1989) e Fisher(1993), fornecem os limites inferior $\theta_{I}$ e superior $\theta_{S}$ para $\tilde{\mu}$. Devido ao pequeno tamanho de amostra, apenas um número limitado de coeficientes de confiança pode ser fixado. Este problema diminui à medida que $n$ aumenta.

Quando $n \geq 16$, podemos obter limites de confiança aproximados para $\tilde{\mu}$ (ver Conover(1971), seção 3.2). Neste caso os limites inferior e superior de confiança para $\tilde{\mu}$ são obtidos contando-se $m$ valores à esquerda de $\tilde{\theta}$ e $m$ à direita de $\tilde{\theta}$ na amostra ordenada. Fixado um coeficiente de confiança $\gamma$, o valor de $m$ é dado por:

$$
m=1+\left[\frac{1}{2} \sqrt{n} z_{\alpha / 2}\right]
$$

onde

$$
\begin{aligned}
& \alpha=1-\gamma \\
& z_{\alpha / 2} \text { é o quantil de ordem } 1-\frac{\alpha}{2} \text { da distribuição } N(0,1) \text { e } \\
& {[.] \text { : indica a parte inteira do valor da expressão. }}
\end{aligned}
$$

\subsubsection{Teste de hipótese sobre a direção mediana}

Suponhamos que desejamos testar as hipóteses:

$$
\begin{array}{ll}
H_{0}: & \tilde{\mu}=\tilde{\mu}_{0} \\
H_{1} & : \quad \tilde{\mu} \neq \tilde{\mu}_{0}
\end{array}
$$

a um nível de significância $\alpha$. Uma forma de efetuar o teste é através da construção do intervalo de confiança para $\tilde{\mu}$. Para $n \geq 16$, o teste também pode ser realizado considerando-se a estatística:

$$
Y=\frac{(2 m-n+k)^{2}}{n-k}
$$


onde

$m$ é o número de dados no $\operatorname{arco}\left(\tilde{\mu}_{0}, \tilde{\mu}_{0}+180^{\circ}\right)$ não iguais a $\tilde{\mu}_{0} \mathrm{e}$ $k$ é o número de valores amostrais iguais a $\tilde{\mu}_{0}$.

Se $\tilde{\mu}_{0}$ é a direção mediana verdadeira, $m$ não deve diferir muito de $(n-k) / 2$.

Rejeitamos $H_{0}$, com $\alpha$ fixado, quando $Y$ é muito grande. Sob $H_{0}$, a distribuição de $Y$ é próxima de uma $\chi_{1}^{2}$. Assim rejeitamos $H_{0}$ se $Y>\chi_{1, \alpha}^{2}$, onde $\chi_{1, \alpha}^{2}$ é o quantil de ordem $1-\alpha$ da distribuição $\chi^{2}$ com 1 grau de liberdade.

\subsubsection{Estimação da direção média}

Um estimador pontual da direção média populacional $\mu$ é dado por $\bar{\theta}$ (ver expressão (2.2)).

A construção de um intervalo de confiança para $\mu$, com coeficiente de confiança $\gamma=1-\alpha$, depende do tamanho da amostra .

Para $n<25$, é recomendado o método bootstrap, descrito na seção B.2 do Apêndice B e proposto por Fisher e Hall(1989).

Para $n \geq 25$, Fisher e Lewis(1983) propuseram o seguinte intervalo de confiança aproximado:

$$
\left[\bar{\theta} \pm \operatorname{arcsen}\left(z_{\alpha / 2} \hat{\sigma}\right)\right]
$$

onde

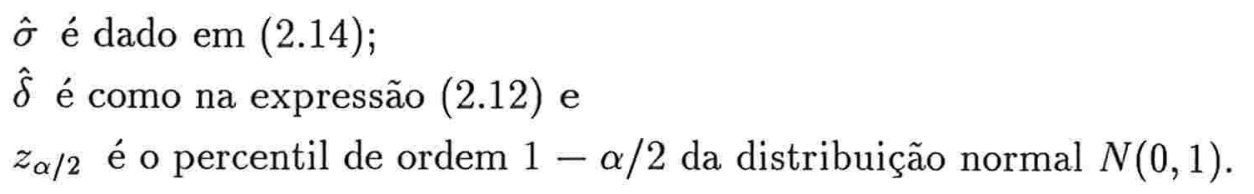

\subsubsection{Teste de hipótese sobre a direção média}

Consideremos o problema de testar as hipóteses:

$$
\begin{aligned}
& H_{0}: \quad \mu=\mu_{0} \\
& H_{1}: \quad \mu \neq \mu_{0},
\end{aligned}
$$


onde $\mu_{0}$ é uma direção fixada, a um nível de significância $\alpha$. Se já tivermos construído um intervalo de confiança para $\mu$ com coeficiente de confiança $\gamma=1-\alpha$, uma forma de testar $H_{0}$ seria através desse intervalo. Se $\mu_{0}$ pertence ao intervalo, não rejeitamos $H_{0}$.

Outra forma de realizar o teste é considerar diretamente uma estatística baseada na distancia entre $\bar{\theta}$ e $\mu_{0}$, dada por:

$$
T=\frac{\operatorname{sen}\left(\bar{\theta}-\mu_{0}\right)}{\hat{\sigma}}
$$

onde

$\hat{\sigma}$ é o erro padrão circular, obtido a partir da expressão (2.14) e $\bar{\theta}$ é a direção média amostral.

Rejeitamos $H_{0}$ para valores grandes de $|T|$.

Para $n<25$ a distribuição da estatística $T$ sob $H_{0}$ é construída utilizando-se o procedimento bootstrap descrito na seção B.3 do Apêndice B. Este procedimento foi sugerido por Fisher e Powell(1989).

Para valores grandes de $n(n \geq 25)$, Watson(1983) mostrou que, sob $H_{0}, T$ tem distribuição próxima da $N(0,1)$. Assim, rejeitamos $H_{0}$ se $|T|>z_{\alpha}$, onde $z_{\alpha}$ é o quantil de ordem $1-\frac{\alpha}{2}$ da distribuição $N(0,1)$.

\subsection{Análise estatística de uma amostra da distri- buição von Mises}

Conforme citamos na Seção 3.1 o modelo de von Mises é o mais utilizado em técnicas paramétricas para a análise de dados circulares. Nesta seção estudaremos a análise de uma amostra da distribuição de von Mises considerando um teste de aderência ao modelo, um procedimento para detectar outliers na amostra, a estimação dos parâmetros do modelo por ponto e intervalo, e testes de hipóteses sobre $\mu$ e $\kappa$. 


\subsubsection{Teste de aderência}

Nesta subseção são descritos um método gráfico e um formal para avaliar se os dados seguem uma distribuição de von Mises.

\section{a) Método gráfico}

Vamos construir um gráfico do tipo $Q-Q$ plot. Para isso, inicialmente precisamos obter as estimativas $\hat{\mu}$ e $\hat{\kappa}$ dos parâmetros $\mu$ e $\kappa$ (ver expresões (2.2) e (4.19)). Calculamos então:

$$
q_{i}=F_{\hat{\kappa}}^{-1}\left(p_{i}\right),
$$

onde

$$
\begin{aligned}
& p_{i}=\frac{i}{(n+1)}, \quad i=1, \ldots, n \text { e } \\
& F_{\hat{\kappa}}^{-1} \text { é a inversa da função de distribuição de uma von Mises } V M(0, \hat{\kappa}),
\end{aligned}
$$

isto é, $F_{\hat{\kappa}}^{-1}(p)=\theta$ significa que $F_{\hat{\kappa}}(\theta)=p, 0 \leq p \leq 1$, ou seja, $\theta$ é o quantil de ordem $p$ de uma von Mises $(0, \hat{\kappa})$. $O$ cálculo de $F_{\hat{\kappa}}^{-1}(p)$ para um dado $p$ não é simples. Um procedimento iterativo para a obtenção de um valor aproximado dessa função é apresentado em Fisher(1993). Calculamos também:

$$
z_{i}=\operatorname{sen} \frac{1}{2}\left(\theta_{i}-\hat{\mu}\right), \quad i=1, \ldots, n,
$$

e os rearranjamos em ordem crescente obtendo $z_{(1)} \leq \ldots \leq z_{(n)}$. Representamos graficamente os pontos:

$$
\left(\operatorname{sen}\left(\frac{1}{2} q_{1}\right), z_{(1)}\right), \ldots,\left(\operatorname{sen}\left(\frac{1}{2} q_{n}\right), z_{(n)}\right) .
$$

Se os dados correspondem a uma amostra de uma distribuição de von Mises, os pontos devem estar dispersos sobre uma reta que passa pela origem com uma inclinação de $45^{\circ}$. Quando a amostra é grande, Fisher(1993) recomenda que, a fim de corrigir instabilidades que podem ocorrer nos extremos do gráfico, sejam calculados: 


$$
z_{i}^{*}=\left|\operatorname{sen} \frac{1}{4}\left(\theta_{i}-\hat{\mu}\right)\right|, \quad i=1, \ldots, n,
$$

e sejam representados graficamente os pontos

$$
\left(\operatorname{sen}\left(\frac{1}{4} q_{1}\right), z_{(1)}^{*}\right), \ldots,\left(\operatorname{sen}\left(\frac{1}{4} q_{n}\right), z_{(n)}^{*}\right) .
$$

b) Teste de aderência

Quando $n \geq 20$, podemos utilizar um procedimento formal para testar a hipótese:

$$
H_{0}: \text { Os dados seguem uma distribuição von Mises. }
$$

$\mathrm{O}$ teste de $H_{0}$ se baseia na função de distribuição acumulada da von Mises. A estatística do teste é dada por:

$$
U^{2}=\sum_{i=1}^{n}\left[z_{(i)}^{2}\right]-\frac{1}{n} \sum_{i=1}^{n}(2 i-1) z_{(i)}+\frac{n}{3}-n\left(\bar{z}-\frac{1}{2}\right)^{2}
$$

onde

$$
\begin{aligned}
& z_{i}=F_{\hat{\kappa}}\left(\theta_{i}-\hat{\mu}\right), \quad i=1, \ldots, n, \\
& \hat{\mu} \text { e } \hat{\kappa} \text { são obtidos das expressões }(4.15) \text { e }(4.19) \mathrm{e} \\
& \bar{z}=\sum_{i=1}^{n} z_{(i)} / n \text { é obtido rearranjando os } z_{i} \text { em ordem crescente, } z_{(1)}<\ldots<z_{(n)} .
\end{aligned}
$$

A distribuição de $U^{2}$ está tabulada em Fischer(1993) e rejeitamos $H_{0}$ para valores grandes de $U^{2}$.

\subsubsection{Teste para outliers}

Ocasionalmente encontramos conjuntos de dados nos quais uma ou mais observações se destacam das demais. Esses valores discrepantes (outliers) podem ocorrer devido a um erro na codificação dos dados, ou à variação amostral ter ocasionado um valor isolado. Exploratoriamente, podemos julgar se um ponto se destaca da massa de dados representando graficamente a amostra em um diagrama circular (Seção $2.2)$, ou através de um $Q$-Qplot, assumindo que os dados foram obtidos a partir de 
um dado modelo probabilístico. Embora a ocorrência de outliers não apresente sérios problemas à análise de dados circulares (Fisher,1993), consideramos nesta Subseção um procedimento formal de teste que permite verificar se um ponto se afasta de forma significativa da massa de dados, quando a amostra provém de uma distribuição de von Mises. Este teste foi descrito por Collett(1980).

Suponha que uma observação $\theta_{k}$ se afasta da massa de dados, e sejam $R$ e $R_{k}$ os comprimentos das resultantes na amostra completa e na amostra obtida excluindo-se $\theta_{k}$, respectivamente. A estatística do teste é dada por:

$$
M_{n}=\frac{R_{k}-R+1}{n-R}, \quad k=1, \ldots, n .
$$

Se $\theta_{k}$ é um valor discrepante a diferença entre $R$ e $R_{k}$ é grande; assim $\theta_{k}$ é classificado como um "outlier" se $M_{n}$ é grande. Valores críticos para o teste são dados en Fisher(1993).

\subsubsection{Estimação de máxima verossimilhança dos parâmetros $\mu$ e $\kappa$}

Seja $\theta_{1}, \ldots, \theta_{n}$ uma amostra de tamanho $n$ de uma distribuição de von $\operatorname{Mises}(\mu, \kappa)$.

A função de verossimilhança das observações é dada por:

$$
L(\mu, \kappa ; \theta)=\left[2 \pi I_{0}(\kappa)\right]^{-n} \exp \left\{\kappa \sum_{i=1}^{n} \cos \left(\theta_{i}-\mu\right)\right\}
$$

Maximizar (4.12) é equivalente a maximizar o logaritmo da função de verossimilhança, ou seja,

$$
\ell(\mu, \kappa)=-n \ln 2 \pi-n \ln I_{0}(\kappa)+\kappa \sum_{i=1}^{n} \cos \left(\theta_{i}-\mu\right)
$$

O estimador de máxima verossimilhança de $\mu, \hat{\mu}$, satisfaz a equação:

$$
\frac{\partial \ell}{\partial \mu}=\hat{\kappa} \sum_{i=1}^{n} \operatorname{sen}\left(\theta_{i}-\hat{\mu}\right)=0 \quad \Longrightarrow \sum_{i=1}^{n} \operatorname{sen}\left(\theta_{i}-\hat{\mu}\right)=0
$$


Da subseção (2.3.1)sabemos que esta equação é satisfeita quando $\hat{\mu}$ é o ângulo médio amostral. Assim, o estimador de máxima verossimilhança de $\mu$ é dado por:

$$
\hat{\mu}=\bar{\theta} \text {. }
$$

O estimador de $\kappa$ é tal que:

$$
\frac{\partial \ell}{\partial \kappa}=-n \frac{I_{0}^{\prime}(\hat{\kappa})}{I_{0}(\hat{\kappa})}+\sum_{i=1}^{n} \cos \left(\theta_{i}-\hat{\mu}\right)=0 .
$$

Considerando a propriedade da função Bessel: $I_{0}^{\prime}(\hat{\kappa})=I_{1}(\hat{\kappa})$, temos:

$$
\frac{I_{1}(\hat{\kappa})}{I_{0}(\hat{\kappa})}=\frac{1}{n} \sum_{i=1}^{n} \cos \left(\theta_{i}-\hat{\mu}\right) .
$$

Da subseção (2.3.1) sabemos que $\sum_{i=1}^{n} \cos \left(\theta_{i}-\bar{\theta}\right)=n r$. Logo, o estimador de máxima verossimilhança de $\kappa$ é a solução de:

$$
A(\hat{\kappa})=\frac{I_{1}(\hat{\kappa})}{I_{0}(\hat{\kappa})}=r .
$$

Mas esta equação não tem uma solução explícita e deve ser resolvida numericamente. Fisher(1993), usando propriedades da função Bessel, apresenta uma aproximação para a solução de (4.18) que é dada por:

$$
\hat{\kappa}=\left\{\begin{array}{lll}
2 r+r^{3}+\frac{5 r^{5}}{6} & \text { se } & r<0,53 \\
-0,4+1,39 r+\frac{0,43}{1-r} & \text { se } & 0,53 \leq r<0,85 \\
\frac{1}{r^{3}-4 r^{2}+3 r} & \text { se } & r \geq 0,85
\end{array}\right.
$$

Infelizmente, quando o tamanho da amostra e $r$ são pequenos $(r<0,45), \hat{\kappa}$ tende a superestimar o parâmetro $\kappa$. Fisher propôs a seguinte estimativa para $\kappa$ quando $n \leq 15$ :

$$
\hat{\kappa}^{*}=\left\{\begin{array}{lll}
\max \left(\hat{\kappa}-2(n \hat{\kappa})^{-1}, 0\right) & \text { se } & \hat{\kappa}<2 \\
\frac{(n-1)^{3} \hat{\kappa}}{n^{3}+n} & \text { se } & \hat{\kappa} \geq 2 .
\end{array}\right.
$$


Propriedades dos estimadores de máxima verossimilhança $\hat{\mu}$ e $\hat{\kappa}$

Para n grande temos que:

$$
\begin{aligned}
E(\hat{\mu}) & =\mu \mathrm{e} \\
\operatorname{var}(\hat{\mu}) & \simeq \frac{1}{n \kappa A(\kappa)} .
\end{aligned}
$$

Utilizando aproximações para $A(\kappa)$, Mardia(1972) mostrou que a expressão (4.22) pode ser escrita como:

$$
\operatorname{var}(\hat{\mu})=\left\{\begin{array}{lll}
\frac{2}{n \kappa^{2}}, & \text { se } & \kappa \leq 10 \\
\frac{1}{n \kappa}, & \text { se } & \kappa>10
\end{array}\right.
$$

Assim $\mu$ pode ser estimado com menor precisão para $\kappa$ pequeno do que para valores grandes de $\kappa$, o que seria esperado, uma vez que, quando $\kappa \rightarrow 0$, a distribuição de von Mises se aproxima da distribuição uniforme circular.

Com relação a $\hat{\kappa}$, temos que:

onde

$$
E(\hat{\kappa}) \simeq \kappa+\frac{1}{2 n B^{2}}\left(2 \kappa^{2} A(\kappa)(1-A(\kappa))-3 A^{2}(\kappa) \kappa+\kappa-2 A(\kappa)\right),
$$

$$
\begin{gathered}
B=\kappa\left(1-A^{2}(\kappa)\right)-A(\kappa), \quad e \\
\operatorname{var}(\hat{\kappa})=\frac{\kappa}{n\left(\kappa-\kappa A^{2}(\kappa)-A(\kappa)\right)} .
\end{gathered}
$$

Notamos que o viés de $\hat{\kappa}$ não é negligível, a menos que $n$ seja grande. Temos ainda que,

$$
\operatorname{cov}(\hat{\mu}, \hat{\kappa})=0 .
$$

Portanto, para $n$ grande, $\hat{\mu}$ e $\hat{\kappa}$ são variáveis aleatórias independentes e

$$
\begin{array}{ll}
\hat{\mu} & \stackrel{D}{\rightarrow} N(\mu, \operatorname{var}(\hat{\mu})) e \\
\hat{\kappa} \stackrel{D}{\rightarrow} N(\kappa, \operatorname{var}(\hat{\kappa})),
\end{array}
$$

onde $\operatorname{var}(\hat{\mu})$ e $\operatorname{var}(\hat{\kappa})$ são dadas por (4.22) e (4.24), respectivamente. Lembremos que, os estimadores de máxima verossimilhança são assintoticamente eficientes. 


\subsubsection{Estimação por intervalo dos parâmetros $\mu$ e $\kappa$}

\section{a) Estimação por intervalo de $\mu$}

A forma de obtenção dos limites de confiança para $\mu$, fixado um coeficiente de confiança $\gamma=1-\alpha$, vai depender do tamanho da amostra e da magnitude de $\hat{\kappa}$.

Um guia para seleção do método a ser utilizado com base aos valores de $n$ e $\hat{\kappa}$ é apresentado na tabela 4.1 .

Para valores muito pequenos de $\hat{\kappa}(\hat{\kappa}<0,4)$, é sugerido que o intervalo seja construído através do procedimento bootstrap paramétrico descrito em B.6.

Para valores grandes de $\hat{\kappa}(\hat{\kappa}>2)$ ou tamanhos de amostras grandes, podem ser utilizados os resultados assintóticos apresentados em Fisher e Lewis(1983). Esses autores mostraram que, para $n$ ou $\kappa$ grandes um intervalo de confiança para $\mu$ é:

$$
\hat{\mu} \pm \operatorname{sen}^{-1}\left(z_{\alpha / 2} \hat{\sigma}_{V M}\right)
$$

onde

$z_{\alpha / 2}$ denota o quantil de ordem $1-\frac{\alpha}{2}$ da distribuição $N(0,1)$ e,

$$
\hat{\sigma}_{V M}=\frac{1}{(n r \hat{\kappa})^{1 / 2}}
$$

Considerando as propriedades assintóticas de $\hat{\mu}$ e $\hat{\kappa}$ apresentadas na seção anterior, temos ainda que, para $n$ grande, um intervalo de confiança para $\mu$ é dado por:

$$
\hat{\mu} \pm z_{\frac{\alpha}{2}} \sqrt{\operatorname{var}(\hat{\mu})} \Longrightarrow \hat{\mu} \pm z_{\frac{\alpha}{2}}(n r \hat{\kappa})^{-1 / 2}
$$

onde $z_{\frac{\alpha}{2}}$ é o quantil de ordem $1-\frac{\alpha}{2}$ da distribuição $N(0,1)$. Como $\operatorname{sen}^{-1} x=x+\frac{x^{3}}{6}+\ldots$, os intervalos (4.25) e (4.27) são essencialmente equivalentes para pequenos valores de $(n r \hat{\kappa})^{-1}$, o que ocorre quando $n$ é grande.

Outros intervalos de confiança para $\mu$ foram propostos por outros autores, entre eles: Mardia(1972), Cabrera(1991) e Upton e Finglenton (1989). 
Tabela 4.1: Guia para seleção do método de estimação

\begin{tabular}{|l|c|c|}
\hline & Método bootstrap & Método assintótico \\
\hline$\hat{\kappa}<0,4$ & $\forall n$ & \\
$0,4 \leq \hat{\kappa}<1,0$ & $n<25$ & $n \geq 25$ \\
$1,0 \leq \hat{\kappa}<1,5$ & $n<15$ & $n \geq 15$ \\
$1,5 \leq \hat{\kappa}<2$ & $n<10$ & $n \geq 10$ \\
$\hat{\kappa} \geq 2,0$ & & $\forall n$ \\
\hline
\end{tabular}

\section{b)Estimação por intervalo de $\kappa$}

Quando $\hat{\kappa}<2$ utilizamos o método bootstrap paramétrico descrito em B.8. Obtendo, através de reamostragem, $B$ valores de $C=\sum \cos \theta_{i}$ e $S=\sum \operatorname{sen} \theta_{i}$ :

$$
\left[\begin{array}{c}
C_{1} \\
S_{1}
\end{array}\right] \cdots\left[\begin{array}{c}
C_{B} \\
S_{B}
\end{array}\right] .
$$

Calculamos $r_{i}^{*}=\left(C_{i}^{2}+S_{i}^{2}\right)^{1 / 2}, \hat{\kappa}_{i}^{*}$ utilizando as expressões (4.19) (ou (4.20)) com $r=r_{i}^{*}$ para $i=1, \ldots, B$. Ordenamos as estimativas bootstraps $\hat{\kappa}_{1}^{*}, \ldots, \hat{\kappa}_{B}^{*}$ em ordem crescente, obtendo $\hat{\kappa}_{(1)}^{*} \leq \ldots \leq \hat{\kappa}_{(B)}^{*}$. Um intervalo de confiança para $\kappa$ com coeficiente de confiança $\gamma=1-\alpha$ é dado por:

$$
\left(\hat{\kappa}_{(l+1)}^{*}, \hat{\kappa}_{(m)}^{*}\right)
$$

onde, $l=\left[\left(\frac{1}{2} B \alpha+\frac{1}{2}\right)\right]$, [.] indica parte inteira e $m=B-l$.

Para $\hat{\kappa} \geq 2$, utilizamos a seguinte aproximação demostrada por Stephen(1969):

$$
2 \psi(n-R) \simeq \chi_{n-1}^{2}
$$

onde

$$
\psi^{-1}=\kappa^{-1}+\frac{3}{8} \kappa^{-2} \text {. }
$$

Um intervalo de confiança para $\kappa$ com coeficiente de confiança $\gamma=(1-\alpha)$ é dado por:

$$
\left[\frac{1+(1+3 a)^{1 / 2}}{4 a}, \frac{1+(1+3 b)^{1 / 2}}{4 b}\right]
$$

onde 
$a=\frac{(n-r)}{\chi_{n-1}^{2}\left(1-\frac{1}{2} \alpha\right)}, b=\frac{(n-r)}{\chi_{n-1}^{2}\left(\frac{1}{2} \alpha\right)}$ e $\chi_{n-1}^{2}(\alpha)$ é o percentil de ordem $1-\alpha$ de uma $\chi_{n-1}^{2}$.

A escolha entre os dois métodos de construção de intervalo de confiança para $\kappa$ descritos acima depende apenas do valor observado $\hat{\kappa}$, que é um indicativo da magnitude do verdadeiro valor do parâmetro. Para amostras grandes, podemos construir um intervalo de confiança utilizando os resultados assintóticos apresentados na seção 4.4.3. Fixando um coeficiente de confiança $\gamma=1-\alpha$, temos que um intervalo de confiança para $\kappa$ é dado por:

$$
\hat{\kappa} \pm z_{\frac{\alpha}{2}} \sqrt{\hat{v} a r(\hat{\kappa})}
$$

onde $z_{\frac{\alpha}{2}}$ é o quantil de ordem $1-\frac{\alpha}{2}$ da distribuição $N(0,1)$ e $\hat{v} a r(\hat{\kappa})$ é a variância estimada de $\hat{\kappa}$ obtida a partir de (4.24) substituindo-se $\kappa$ por $\hat{\kappa}$.

\subsubsection{Testes de hipótese sobre $\mu$ e $\kappa$}

\section{a) Teste de hipótese sobre $\mu$}

Suponha que desejamos testar as hipóteses:

$$
\begin{aligned}
& H_{0}: \quad \mu=\mu_{0} \\
& H_{1}: \mu \neq \mu_{0},
\end{aligned}
$$

onde $\mu_{0}$ é uma constante conhecida. Suponhamos que o parâmetro de concentração $\kappa$ é desconhecido. Uma forma de testar $H_{0}$ é através da construção do intervalo de confiança para $\mu$ (ver subseção 4.4.4(a)); se $\mu_{0}$ está neste intervalo se aceita a hipótese nula.

Outra forma de testar $H_{0}$ (4.30) é através da estatística:

$$
T_{n}=\frac{\left[\operatorname{sen}\left(\hat{\mu}-\mu_{0}\right)\right]}{(n r \hat{\kappa})^{-\frac{1}{2}}} .
$$

Para valores grandes de $n$ ou $\hat{\kappa},\left(\right.$ Fisher e Lewis (1983)) $T_{n}$ tem distribuição próxima a uma $N(0,1)$. Assim, rejeitamos $H_{0}$ a um nível aproximadamente igual $\alpha$ se $\left|T_{n}\right|>z_{\frac{\alpha}{2}}$, onde $z_{\frac{\alpha}{2}}$ denota o quantil de ordem $1-\alpha / 2$ da distribuição normal padrão. Para valores pequenos de $n$ e $\hat{\kappa}$ a distribuição da estatística é gerada pelo método bootstrap (ver B.7). A Tabela 4.1 serve como guia para a escolha do método de teste a ser adotado. 
b) Teste de hípotese sobre $\kappa$

Suponhamos que desejamos testar as seguintes hipóteses:

$$
\begin{aligned}
& H_{0}: \quad \kappa=\kappa_{0} \\
& H_{1}: \quad \kappa \neq \kappa_{0},
\end{aligned}
$$

considerando que $\mu$ é desconhecido. Da mesma forma como em a) podemos utilizar o intervalo de confiança para $\kappa$ para testar as hipóteses acima. Quando $\kappa$ é grande $(\hat{\kappa}>2)$ podemos também realizar o teste diretamente, considerando a estatística :

$$
R=n A(\hat{\kappa}) .
$$

Rejeitamos $H_{0}$ ao nível $\alpha$ quando:

$$
R<n-\chi_{n-1}^{2}\left(\frac{\alpha}{2}\right) / 2 \psi_{0}
$$

ou

$$
R<n-\chi_{n-1}^{2}\left(1-\frac{\alpha}{2}\right) / 2 \psi_{0}
$$

onde, $\psi_{0}=\frac{1}{\frac{1}{\kappa_{0}}+\frac{3}{8 \kappa_{0}^{2}}}$. 


\section{Capítulo 5}

\section{Técnicas não paramétricas para duas ou mais amostras}

\subsection{Introdução}

Neste capítulo consideraremos a análise de experimentos completamente casualizados com um fator fixo (ver, por exemplo, Neter, Wasserman e Kutner(1996)), nos quais a variável resposta é circular. Nestes experimentos, tem-se interesse em responder a uma ou mais das seguintes questões: as amostras provêm de distribuições com a mesma direção de referência (direção média ou mediana)? Em caso positivo, como combiná-las de forma a obter uma estimativa comum desse parâmetro de locação? O que podemos dizer sobre as dispersões das distribuições? As distribuições podem, como um todo, ser consideradas idênticas?

Embora considerável teoria estatística tenha sido desenvolvida para as respostas a essas questões quando se tem duas ou mais amostras de distribuições de von Mises, esta distribuição pode não fornecer uma descrição adequada do conjunto de dados a ser analisado. Assim torna-se também necessário o conhecimento de métodos não paramétricos. Segundo Rao(1984), a importância desses métodos na análise de dados circulares é maior que para dados na reta. Em inferência linear (na reta) existem situações nas quais é natural a suposição de normalidade, como, por exemplo, quando trabalhamos com médias, ou quando as amostras são suficientemente grandes. Infelizmente, não existem, para dados circulares, situações semelhantes que justifiquem a suposição de que as amostras provêm de distribuições de von Mises. 
Antes da decisão pela aplicação de técnicas paramétricas ou não paramétricas, é recomendável um estudo descritivo detalhado das observações. Os procedimentos descritos no capítulo 2 podem ser aplicados às amostras individualmente. A análise descritiva nos indica se as distribuições que geraram os dados são simétricas, unimodais, se diferem apenas quanto à direção de referência, ou se suas dispersões devem também ser comparadas. O teste de uniformidade descrito na seção 4.2 também deve ser aplicado. Excluiremos de análises posteriores conjuntos de dados que tenham sido obtidos de uma distribuição circular uniforme. A verificação de que as amostras foram retiradas de uma população com distribuição de von Mises pode ser feita através dos procedimentos apresentados na seção 4.4.1. Caso o modelo de von Mises seja adequado, as técnicas de inferência estatística apresentadas no capítulo 6 devem ser adotadas.

Assumindo apenas que os dados a serem analisados correspondem a amostras de distribuições circulares unimodais contínuas, apresentamos neste capítulo técnicas não paramétricas para a análise de experimentos com um fator fixo. Na seção 5.2 apresentamos inferências sobre as direções medianas, e na seção 5.3, inferências sobre as direções médias. Um teste para a comparação das dispersões de duas populações é apresentado na seção 5.4. Testes de homogeneidade de duas ou mais distribuições são tratados na seção 5.5. Os procedimentos descritos nas seções 5.2 a 5.5 se aplicam diretamente a dados vetoriais. Na seção 5.6 mostramos como essas técnicas podem ser aplicadas a dados axiais.

Adotaremos a seguinte notação. Sejam:

- $\theta_{i j}, i=1, \ldots, q, j=1, \ldots, n_{i}$, amostras aleatórias independentes de tamanhos $n_{1}, \ldots, n_{q}$ de funções de densidade contínuas $f_{i}(\theta)$

- $n=\sum_{i=1}^{q} n_{i}$

- $\bar{\theta}_{i}$ : média amostral na i-ésima amostra;

- $\bar{C}_{i}=\sum_{j=1}^{n_{i}} \cos \theta_{i j} / n_{i} ; \bar{S}_{i}=\sum_{j=1}^{n_{i}} \operatorname{sen} \theta_{i j} / n_{i}$;

- $r_{i}^{2}=\left(\bar{C}_{i}^{2}+\bar{S}_{i}^{2}\right)$;

- $r_{2 i}=\left[\left(\frac{1}{n_{i}} \sum_{j=1}^{n_{i}} \cos 2\left(\theta_{i j}-\bar{\theta}\right)\right)^{2}+\left(\frac{1}{n_{i}} \sum_{j=1}^{n_{i}} \operatorname{sen} 2\left(\theta_{i j}-\bar{\theta}_{r}\right)\right)^{2}\right]^{1 / 2}$;

- $\hat{\delta}_{i}=\left(1-r_{2 i}\right) / 2 r_{i}^{2} \quad \mathrm{e}$ 
- $\hat{\sigma}_{i}=\left(\hat{\delta}_{i} / n_{i}\right)^{1 / 2}$.

\subsection{Inferências sobre as direções medianas de duas ou mais populações circulares}

Se a análise preliminar dos dados indicar que as amostras são provenientes de distribuições unimodais, é interessante compararmos suas "direções de preferência", isto é, suas direções médias ou direções medianas. Segundo Fisher(1993), a direção média, em geral, é mais utilizada em problemas práticos como "direção de preferência". Entretanto, a direção mediana é útil em aplicações nas quais se observa assimetria da distribuição, e inferências sobre essa direção vão ser consideradas nesta seção. Na subseção 5.2.1 apresentamos um teste para a comparação de medianas em populações circulares deduzido por Fisher e Powell(1989), e na subseção 5.2.2 mostramos como estimar a direção mediana comum de duas ou mais populações.

\subsubsection{Teste de igualdade de duas ou mais direções medianas}

Suponhamos que estamos interessados em testar

$$
H_{0}: \tilde{\mu}_{1}=\ldots=\tilde{\mu}_{q},
$$

onde $\tilde{\mu}_{i}$ é direção mediana. Este teste requer $n_{i} \geq 10$, a menos que todos os tamanhos de amostra $n_{1}, \ldots, n_{q}$ sejam aproximadamente iguais. O procedimento a ser adotado é: calculamos a direção mediana geral $\tilde{\theta}$ na amostra combinada com $n$ dados. Para cada $i=1,2, \ldots, q$, seja $m_{i}$ o número de valores negativos de $\theta_{i 1}-\tilde{\theta}, \ldots, \theta_{i n_{i}}-\tilde{\theta}$. Notemos que $\theta_{i j}-\tilde{\theta}$ assume valores no intervalo $[-\pi, \pi]$. Seja $M=m_{1}+\ldots+m_{q}$. A estatística para o teste é,

$$
P_{q}=\left[\frac{n^{2}}{M(n-M)}\right] \sum_{i=1}^{q} \frac{m_{i}^{2}}{n_{i}}-\frac{n M}{n-M} .
$$

Sejam $\hat{p}=\frac{M}{n}$ a proporção de negativos na amostra geral de tamanho $n$ e $\hat{p}_{i}=\frac{m_{i}}{n_{i}}$ a proporção de negativos na i-ésima amostra, $i=1, \ldots, q$. Se as medianas populacionais são iguais, então, devemos esperar que $\hat{p}_{i} \simeq \ldots \simeq \hat{p}_{q} \simeq \hat{p}$. Neste caso, observamos que a expressão (5.1), converte-se em: 
$P_{q}=\left[\frac{1}{\hat{p}(1-\hat{p})}\right] \sum_{i=1}^{q} m_{i} \hat{p}_{i}-\frac{M}{1-\hat{p}}=\frac{1}{1-\hat{p}}\left[\frac{1}{\hat{p}} \sum_{i=1}^{q} m_{i} \hat{p}-M\right]=\frac{1}{1-\hat{p}}\left[\sum_{i=1}^{q} m_{i}-M\right] \simeq 0$

A hipótese de que as $q$ direções medianas são iguais é então rejeitada para valores grandes de $P_{q}$. Fixado um nível de significância $\alpha$, rejeitamos $H_{0}$ se $P_{q}>\chi_{q-1,1-\alpha}^{2}$, onde $\chi_{q-1,1-\alpha}^{2}$ é o quantil de ordem $1-\alpha$ da distribuição $\chi^{2}$ com $q-1$ graus de liberdade.

\subsubsection{Estimação da direção mediana comum de duas ou mais populações}

Se não rejeitamos a hipótese de que as direções medianas populacionais são iguais, é interessante estimarmos a mediana comum $\tilde{\mu}$ por ponto e por intervalo. A obtenção de limites de confiança para $\tilde{\mu}$ não é trivial, a menos que tenhamos evidência de que todas as $q$ amostras provêm da mesma distribuição. Considerando este caso particular, a estimativa de $\tilde{\mu}$ por ponto é obtida agrupando-se as $q$ amostras em uma única amostra de tamanho $n=n_{1}+\ldots+n_{q}$ e adotando-se o procedimento para o cálculo da mediana em uma amostra apresentado na seção 4.3.1.

\subsection{Inferências sobre as direções médias de duas ou mais populações circulares}

Em amostras de dados na reta, a média e a mediana podem assumir valores muito diferentes devido à presença de valores aberrantes. Segundo Fisher(1993), este fato ocorre com menos frequência em dados circulares; no caso linear as caudas das distribuições podem se estender indefinidamente, mas isto claramente não ocorre no caso circular. Este é um dos motivos pelos quais a direção média amostral é, em geral, adotada como "direção de preferência"; particularmente, em amostras de tamanho moderado a grande, a direção média é mais fácil de ser calculada e, o que é mais importante, combinada com uma medida de dispersão dada pelo comprimento do vetor média, fornece um resumo adequado para os dados estudados. Nesta seção tratamos de inferências sobre as direções médias de $q$ populações circulares, apresentando em 
5.3.1 um teste para a igualdade de direções médias e em 5.3.2 a estimação da direção média comum de $q$ populações.

\subsubsection{Teste da igualdade de duas ou mais direções médias}

Nesta seção consideramos o problema de testar a igualdade de direções médias de duas ou mais distribuições circulares unimodais contínuas. Assim, nossa hipótese de interese é:

$$
H_{0}: \mu_{1}=\ldots=\mu_{q}
$$

onde $q \geq 2$.

Muitos testes foram desenvolvidos com o objetivo de comparar direções médias, especialmente para o caso de duas populações(ver, por exemplo, Batschelet (1981), Mardia (1972), ou Upton e Fingleton (1989)). Entretanto, a significância desses testes não implica que exista algum tipo de diferença entre as direções médias. A significância pode ter sido causada, por exemplo, pelas dispersões das populações não serem todas iguais. Assim esses testes são mais adequados à comparação das populações com um todo. Fisher(1993) apresenta procedimentos específicos para a comparação de direções médias. A escolha do procedimento a ser adotado depende das dispersões amostrais serem ou não "próximas"e dos tamanhos das amostras. As dispersões amostrais $\hat{\delta}_{i}, i=1, \ldots, q$, são consideradas próximas, se a maior delas $\hat{\delta}_{\max }$ não é maior que 4 vezes a menor $\hat{\delta}_{\text {min }}$, isto é, $\hat{\delta}_{\max } \leq 4 \hat{\delta}_{\min }$. (Fischer(1993)).

Quando as dispersões são próximas, utilizamos, para comparar as médias, o método $P$ descrito a seguir. Caso contrário adotamos o método $M$.

Método P.Sejam:

$$
\begin{gathered}
\hat{C}_{p}=\sum_{i=1}^{q} n_{i} \cos \hat{\mu}_{i}, \quad \hat{S}_{p}=\sum_{i=1}^{q} n_{i} \operatorname{sen} \hat{\mu}_{i} \quad e \\
R_{p}=\left(\hat{C}_{p}^{2}+\hat{S}_{p}^{2}\right)^{1 / 2} .
\end{gathered}
$$

Seja $\hat{\delta}_{0}$ a média ponderada das dispersões amostrais $\hat{\delta}_{i}, i=1, \ldots, q$, isto é,

$$
\hat{\delta}_{0}=\sum_{i=1}^{q} \frac{n_{i} \hat{\delta}_{i}}{n} .
$$


A estatística para o teste de $H_{0}$ é:

$$
Y_{q}=2\left(n-R_{p}\right) / \hat{\delta}_{0}
$$

Notemos que, se $H_{0}$ é verdadeira, devemos esperar que,

$$
\hat{\mu}_{1} \simeq \ldots \simeq \hat{\mu}_{q}=\hat{\mu} .
$$

Neste caso,

e

$$
\hat{C}_{p} \simeq \sum_{i=1}^{q} n_{i} \cos \hat{\mu}=n \cos \hat{\mu}
$$

$$
\hat{S}_{p} \simeq n \operatorname{sen} \hat{\mu}
$$

Portanto,

$$
R_{p} \simeq\left(n^{2} \cos ^{2} \hat{\mu}+n^{2} \operatorname{sen}^{2} \hat{\mu}\right)^{1 / 2}=n\left(\operatorname{sen}^{2} \hat{\mu}+\cos ^{2} \hat{\mu}\right)^{1 / 2}=n
$$

Assim, se $H_{0}$ é verdadeira, podemos esperar que a estatística $Y_{q}$ assuma valor próximo de zero. Portanto, rejeitamos $H_{0}$ para valores grandes de $Y_{q}$.

Watson(1983), mostrou que, quando $n_{i} \rightarrow \infty, i=1, \ldots q, Y_{q}$ tem distribuição próxima a uma $\chi_{q-1}^{2}$. Fisher(1993) sugere que, quando $n_{i} \geq 25, i=1, \ldots, q$, seja utilizada a distribuição assintótica de $Y_{q}$ na realização do teste. Se algum $n_{i}<25$, então a distribuição de $Y_{q}$ deve ser construída utilizando-se os métodos de reamostragem descritos nas Seção B.4.

Método M. Sejam:

$$
\hat{C}_{M}=\sum_{i=1}^{q} \frac{\cos \hat{\mu}_{i}}{\hat{\sigma}_{i}^{2}}, \quad \hat{S}_{M}=\sum_{i=1}^{q} \frac{\operatorname{sen} \hat{\mu}_{i}}{\hat{\sigma}_{i}^{2}} \quad, \quad R_{M}=\left(\hat{C}_{M}^{2}+\hat{S}_{M}^{2}\right)^{1 / 2} \text { e } \hat{\sigma}_{i}^{2}=\frac{\hat{\delta}_{i}}{n_{i}} .
$$

A estatística para o teste é :

$$
Y_{q}=2\left(\sum_{i=1}^{q} \frac{1}{\hat{\sigma}_{i}{ }^{2}}-R_{M}\right)
$$

A hipótese de uma direção média comum é rejeitada para valores grandes de $Y_{q}$. A determinação da região crítica do teste é feita de forma análoga à do método $P$. 


\subsubsection{Estimação da direção média comum de duas ou mais distribuições}

Suponhamos que, por algum dos procedimentos descritos na seção anterior, não tenhamos obtido evidências para rejeitar $H_{0}$ dada em (5.2). Nosso objetivo agora é mostrar como obter uma estimativa conjunta $\hat{\mu}_{w}$ da direção média comum $\mu$, a partir dos valores amostrais $\hat{\mu}_{1}, \ldots, \hat{\mu}_{q}$. Se todas as amostras procedem da mesma distribuição, combinamos todos os dados e analisamos a amostra combinada como na subseção 4.3.3. Caso contrário consideramos as combinações lineares dos $\operatorname{sen}\left(\hat{\mu}_{i}\right)$ e $\cos \left(\hat{\mu}_{i}\right), i=1, \ldots, q$, dadas por:

$$
\bar{C}_{w}=\sum_{i=1}^{q} w_{i} r_{i} \cos \hat{\mu}_{i}=\sum_{i=1}^{q} \frac{w_{i} C_{i}}{n_{i}} \text { e } \bar{S}_{w}=\sum_{i}^{q} w_{i} r_{i} \operatorname{sen} \hat{\mu}_{i}=\sum_{i=1}^{q} \frac{w_{i} S_{i}}{n_{i}},
$$

onde $r_{i}, C_{i}$ e $S_{i}$ são, respectivamente, o comprimento médio da resultante e as somas de cosenos e senos na i-ésimas amostra, $i=1, \ldots, q$, e $w_{i}$ são pesos positivos tais que $\sum_{i=1}^{q} w_{i}=1$. Seja ainda:

$$
\bar{\rho}_{w}=\sum_{i}^{q} w_{i} r_{i} .
$$

Fixados os pesos $w_{i}, \hat{\mu}_{w}$ pode ser obtida pela expressão (2.2), utilizando-se $\bar{C}_{w}$ e $\bar{S}_{w}$ no lugar de $C$ e $S$. O erro padrão circular amostral $\left(\hat{\sigma}_{w}\right)$ associado a $\hat{\mu}_{w}$ é obtido a partir de:

$$
\hat{\sigma}_{w}^{2}=\sum_{i=1}^{k} \frac{w_{i}^{2} r_{i}^{2} \hat{\sigma}_{i}^{2}}{\bar{\rho}_{w}^{2}}
$$

$\hat{\sigma}_{i}^{2}$ como na expressão (4.10). O problema passa a ser a determinação dos pesos $w_{i}$ adequados às diferentes situações experimentais. Fisher(1993) sugeriu que a escolha do conjunto de pesos se baseie nas dispersões das $q$ amostras. Se as dispersões amostrais $\hat{\delta}_{1}, \ldots, \hat{\delta}_{q}$ são "próximas", isto é, a maior delas, $\hat{\delta}_{\text {max }}$, não é superior a 4 vezes a menor, $\hat{\delta}_{\min }$, os pesos $w_{i}$ não consideram a dispersão interna das amostras e são dados por:

$$
w_{i}=\frac{n_{i}}{n}, \quad i=1, \ldots, q .
$$

A direção média comum obtida utilizando-se esses pesos em (5.5) coincide com a direção média da amostra de tamanho $n=n_{1}+\ldots+n_{q}$ constituída pelo agrupamento das $q$ amostras originais.

Se $\hat{\delta}_{\max } \geq 4 \hat{\delta}_{\min }$, sejam: 


$$
v_{i}=\left(r_{i} \hat{\sigma}_{i}^{2}\right)^{-1}, \quad i=1, \ldots, q \text { e } \quad v=\sum_{i=1}^{q} v_{i} .
$$

Os pesos $w_{i}$ são agora dados por:

$$
w_{i}=\frac{v_{i}}{v}, \quad i=1, \ldots, q
$$

Qualquer que seja o conjunto de pesos escolhido, para a construção de um intervalo de confiança para $\mu$, adotamos o seguinte procedimento: se algum dos $n_{i}<25$, devem ser utilizadas as técnicas bootstrap propostas por Fisher e Hall(1989) que estão descritas nas seção B.5 do Apêndice B; para grandes amostra, Fisher e Lewis(1983) mostraram que um intervalo de confiança para $\mu$ com coeficiente de confiança $\gamma=$ $1-\alpha$, é dado por:

$$
\hat{\mu}_{w} \pm \operatorname{arcsen}\left(z_{\alpha / 2} \hat{\sigma}_{w}\right)
$$

onde $\hat{\sigma}_{w}$ é o erro padrão circular dado na expressão (5.6), e $z_{\alpha / 2}$ é o quantil de ordem $1-\frac{\alpha}{2}$ da distribuição $N(0,1)$.

\subsection{Teste de igualdade das dispersões de duas ou mais distribuições}

\section{a) Duas amostras}

Suponhamos que estamos interessados em comparar as dispersões de duas populações em relação a determinadas direções de referência $\alpha_{1}$ e $\alpha_{2}$, respectivamente. Essas direções podem ser ângulos pré-fixados que tenham algum significado aplicado no problema em estudo, como por exemplo, a direção do pombal em experimentos com pombos, ou podem ser alguma medida de locação estimada a partir das amostras. Sejam $\theta_{11}, \ldots, \theta_{1 n_{1}}$ e $\theta_{21}, \ldots \theta_{2 n_{2}}$ amostras de tamanho $n_{1}$ e $n_{2}$ de cada uma das populações. Nossa hipótese de interese é:

$H_{0}$ : As dispersões nas duas populações são iguais

$H_{1}$ : As dispersões nas duas populações são diferentes. 
Walbraf(1979) propôs que sejam calculados os desvios :

$$
x_{i j}=\left|\theta_{i j}-\alpha_{i}\right|, \quad i=1,2 \quad j=1, \ldots, n_{i}
$$

Valores de $x_{i j}$ maiores que $180^{\circ}$ devem ser substituidos por $\left(360^{\circ}-x_{i j}\right), i=$ $1,2, j=1, \ldots, n_{i}$. Os desvios obtidos devem ser agrupados em uma única amostra, e atribuir-se postos às observações em ordem crescente. Se as populações possuem dispersões diferentes, espera-se que os postos atribuídos às observações das duas amostras sejam bastante diferentes. Para testar se existe diferença significante, pode ser aplicado o teste de Mann - Whitney (ver, por exemplo, Conover(1971)). Seja $S$ a soma dos postos das observações na primeira amostra. A estatística para o teste é dado por:

$$
U=S-\frac{n(n+1)}{2} .
$$

Rejeitamos $H_{0}$ ao nível de significância $\alpha$, se $U$ é menor ou igual ao quantil de ordem $\alpha / 2$ ou maior que o quantil de ordem $1-\frac{\alpha}{2}$ da distribuição da estatística $U$, sob $H_{0}$ ( ver tabelas em Conover,1971).

Notemos que, no lugar dos desvios $x_{i j}$ definidos acima, podemos considerar:

$$
x_{i j}^{\prime}=\cos \left(\theta_{i j}-\alpha_{i}\right), \quad i=1,2
$$

isto é, a projeção retangular no eixo que passa pela direção $0^{0}$. Os postos de $x_{i j}^{\prime}$ na amostra conjunta são idênticos aos atribuídos a $x_{i j}, i=1,2, j=1, \ldots, n_{i}$. Portanto, para compararmos as dispersões de 2 populações é indiferente trabalharmos com os desvios absolutos ou com os cosenos dos desvios.

\section{b) Mais de duas amostras}

Para comparar as dispersões de mais de duas populações, utilizamos o teste proposto por Rao(1966). Uma medida de concentração na i-ésima amostra é dada por:

$$
r_{i}^{2}=\bar{C}_{i}^{2}+\bar{S}_{i}^{2}, \quad i=1, \ldots, q .
$$

A variância assintótica de $r_{i}^{2}$ é dada por:

$$
s_{i}^{2}=\frac{4}{n_{i}}\left\{\bar{C}_{i}^{2} S_{c c i}+\bar{S}_{i}^{2} S_{s s i}+2 \bar{C}_{i} \bar{S}_{i} S_{c s i}\right\}
$$


onde

$$
\begin{aligned}
& S_{c c i}=\frac{1}{n_{i}} \sum_{j=1}^{n_{i}}\left(\cos \theta_{i j}-\bar{C}_{i}\right)^{2}, \\
& S_{s s i}=\frac{1}{n_{i}} \sum_{j=1}^{n_{i}}\left(\operatorname{sen} \theta_{i j}-\bar{S}_{i}\right)^{2} \mathrm{e} \\
& S_{c s i}=\frac{1}{n_{i}} \sum_{j=1}^{n_{i}}\left(\cos \theta_{i j}-\bar{C}_{i}\right)\left(\operatorname{sen} \theta_{i j}-\bar{S}_{i}\right) .
\end{aligned}
$$

Sob a hipótese de igualdade de dispersões, todas as estatística $r_{1}^{2}, \ldots, r_{q}^{2}$ são independentes e estimadores consistentes dos parâmetros de concentração comum. Uma medida de variabilidade entre os $r_{i}^{2}$ é dada pela estatística:

$$
H=\sum_{1}^{q} \frac{\left(r_{i}^{2}\right)^{2}}{s_{i}^{2}}-\left(\sum_{1}^{q} \frac{r_{i}}{s_{i}^{2}}\right)^{2} /\left(\sum_{1}^{q} \frac{1}{s_{i}^{2}}\right)
$$

que tem distribuição assintótica $\chi^{2} \operatorname{com} q-1$ graus de liberdade, sob a hipótese de igualdade de dispersões. Rejeitamos a hipótese para valores grandes de $H$.

\subsection{Teste de igualdade de duas ou mais distribuições}

Na seção 5.3.1 consideramos o problema de testar se duas ou mais distribuições circulares tinham a mesma direção de referência. Entretanto, em algumas situações, podemos ter interesse em testar a homogeniedade dessas distribuições.

Para a comparação de duas ou mais distribuições circulares, existem varios testes não paramétricos propostos na literatura (ver, por exemplo, Batschelet (1981),Mardia (1972) ou Upton e Fingleton (1989)). O procedemento mais simples consiste no teste $\chi^{2}$ para homogeneidade de duas distribuições, que é bastante conhecido e, por este motivo, não será apresentado neste texto. Observemos somente que, para aplicação deste teste, os dados podem estar agrupados em intervalos de classe. O procedimento descrito com maior frequência é o teste dos escores uniformes. Este teste foi proposto por Wheeler e Watson(1964) e é um caso particular do teste para a comparação de duas populações bivariadas desenvolvidos independentemente por Mardia (1967). A relação entre os dois testes foi mostrada por Mardia(1969). Este estudo tornou o teste aplicável, uma vez que ficou estabelecida a distribuição de sua estatística sob 
a hipótese nula. O teste possui a propriedade de invariância quanto a rotações, isto é, sua estatística não depende da direção fixada como zero, nem dos ângulos serem medidos no sentido horário ou anti-horário. É aplicável a dados não agrupados.

\subsubsection{O teste dos escores uniformes}

\section{a) Duas amostras}

Sejam $\theta_{11}, \ldots, \theta_{1 n_{1}}$ e $\theta_{21}, \ldots, \theta_{2 n_{2}}$ amostras independentes de duas populações contínuas com funções de distribuição $F_{1}(\theta)$ e $F_{2}(\theta)$, respectivamente. Seja $n=n_{1}+n_{2}$. Nosso objetivo é testar:

$$
\begin{array}{ll}
H_{0}: & F_{1}(\theta)=F_{2}(\theta) \\
H_{1} & : \quad F_{1}(\theta) \neq F_{2}(\theta) .
\end{array}
$$

Consideremos a representação das observações das duas amostras em um único círculo de raio unitário. $\mathrm{Na}$ amostra combinada dos $n=n_{1}+n_{2}$ valores observados, podemos associar à observação $\theta_{i j}$ seu posto (ou escore) linear $p_{i j}$ e o correspondente escore circular $\gamma_{i j}=\left(2 \pi p_{i j}\right) / n, j=1, \ldots, n_{i}$ e $i=1,2$. Por exemplo, na Tabela 5.1 representamos os escores lineares e circulares correspondentes a duas amostras.

Tabela 5.1: Observações, postos e escores circulares em duas amostras

\begin{tabular}{||l|l|l||}
\hline \multirow{3}{*}{ amostra 1 } & $\theta_{1 j}$ & $75 ; 75 ; 80 ; 80 ; 80 ; 95 ; 130 ; 170 ; 210$ \\
& $p_{1 j}$ & $6 ; 7 ; 8 ; 9 ; 10 ; 12 ; 13 ; 14 ; 15$ \\
& $\gamma_{1 j}$ & 113,$7 ; 132,6 ; 151,6 ; 170,5 ; 189,5 ; 227,4 ; 246,3 ; 265,3 ; 284,22$ \\
\hline \multirow{3}{*}{ amostra 2 } & $\theta_{2 j}$ & $10 ; 50 ; 55 ; 55 ; 65 ; 90 ; 285 ; 285 ; 325 ; 355$ \\
& $p_{2 j}$ & $1 ; 2 ; 3 ; 4 ; 5 ; 11 ; 16 ; 17 ; 18 ; 19$ \\
& $\gamma_{2 j}$ & 18,$9 ; 37,9 ; 56,8 ; 75,8 ; 94,7 ; 208,4 ; 303,2 ; 322,1 ; 341,1 ; 360$ \\
\hline
\end{tabular}

Representando os escores $\gamma_{i j}$ em um círculo de raio unitário, temos que as distâncias entre pontos sucessivos têm comprimento $(2 \pi) / n$ ( ver Figura 5.1). Se os pontos de uma amostra são suficientemente separados dos pontos da outra amostra, podemos esperar que $F_{1}(\theta)$ seja diferente de $F_{2}(\theta)$. Sejam $R_{1}$ e $R_{2}$ os comprimentos das resultantes na primeira e segunda amostra, repectivamente, e $R$ o comprimento da resultante na amostra combinada, obtidos considerando-se os escores circulares $\gamma_{i j}$. 
Devido à distribuição uniforme desses escores, temos que $R=0$, ou, mais formalmente, como

$$
\sum_{i=1}^{2} \sum_{j=1}^{n_{i}} \frac{\cos 2 \pi p_{i j}}{n}=\sum_{i=1}^{2} \sum_{j=1}^{n_{i}} \frac{\operatorname{sen} 2 \pi p_{i j}}{n}=0,
$$

então $R=0$. Portanto $R_{1}=R_{2}$. Quanto maior for $R_{1}$ (ou $R_{2}$ ), mais separadas são as amostras. Assim, rejeitamos $H_{0}$ para valores grandes de $R_{1}$, ou equivalentemente $R_{1}^{2}$.

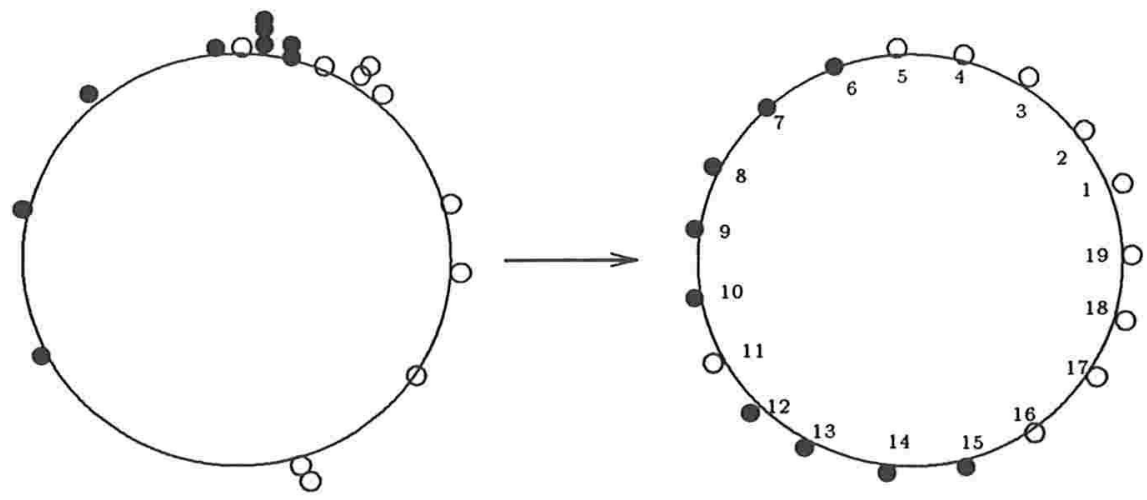

Figura 5.1: Geração de pontos amostrais equidistantes através do cálculo dos escores circulares na amostra combinada.

Mardia(1972) fornece alguns valores críticos para a estatística $R_{1}^{2}$, quando $n \leq 20$. De uma forma geral, esses valores podem ser determinados através de um procedimento de aleatorização como o descrito em Fisher(1993). Sob hipótese nula, todos $n=n_{1}+n_{2}$ pontos foram retirados da mesma distribuição, de modo que a alocação dos valores observados à primeira e à segunda amostra deve-se puramente ao acaso. Existem $\left(\begin{array}{c}n_{1}+n_{2} \\ n_{1}\end{array}\right)=N$ possíveis formas de alocar $n$ pontos a duas amostras de tamanhos $n_{1}$ e $n_{2}$, cada qual com a mesma chance de ocorrência, sob $H_{0}$. Assim, o valor $R_{1}=R_{10}$ obtido no conjunto de dados observados, poderia muito bem ser qualquer um dos outros $N-1$ valores observados nas outras possíveis amostras. Estamos interessados em saber: $R_{1 o}$ é um valor extremo? Para responder a esta pergunta calculamos os $N$ possíveis valores de $R_{1}$ e os ordenamos em ordem crescente: $R_{1(1)} \leq \ldots \leq R_{1(N)}$. Fixando um nível de significância $\alpha$ para o teste, seja:

$$
\ell_{\alpha}=[N \alpha]
$$


onde [.] indica maior inteiro contido, e

$$
m_{\alpha}=N-\ell_{\alpha}+1
$$

Rejeitamos $H_{0}$ se $R_{1 o}>R_{1\left(m_{\alpha}\right)}$.

Para $n>20$ podemos utilizar o resultado de que a estatística

$$
R^{*}=\frac{2(n-1) R_{1}^{2}}{n_{1} \cdot n_{2}}
$$

é aproximadamente $\chi^{2}$ com 2 graus de liberdade, sob $H_{0}$.

\section{b) Generalização para mais de duas amostras}

Podemos estender o procedimento descrito em a) da seguinte forma:

Sejam $\theta_{i j}, j=1, \ldots, n_{i}, i=1, \ldots, q, q$ amostras independentes de tamanhos $n_{1}, \ldots, n_{q}$ de funções distribuições contínuas. Nosso objetivo é testar :

$$
\begin{aligned}
& H_{0}: F_{1}(\theta)=\ldots=F_{q}(\theta) \\
& H_{1}: \text { pelo menos uma das distribuições é diferente das demais. }
\end{aligned}
$$

Procedemos de forma análoga ao caso de duas populações: agrupamos $n=n_{1}+$ $\ldots+n_{q}$ observações em uma única amostra combinada, obtemos seus postos lineares $r_{i j}$ e os correspondentes escores circulares $\gamma_{i j}=\left(2 \pi p_{i j}\right) / n, j=1, \ldots, n_{i}$ e $i=1, \ldots, q$. Sejam:

$$
C_{i}=\sum_{j=1}^{n_{i}} \cos \gamma_{i j} \text { e } S_{i}=\sum_{j=1}^{n_{i}} \operatorname{sen} \gamma_{i j}
$$

A estatística para o teste é:

$$
W=2 \sum_{i=1}^{q}\left(C_{i}^{2}+S_{i}^{2}\right) / n_{i}=2 \sum_{i=1}^{q} \frac{R_{i}^{2}}{n_{i}} .
$$

Notemos que, para $q=2, W=\left(2 n R_{1}^{2}\right) / n_{1} n_{2} \simeq R^{*}\left(R^{*}=2(n-1) R_{1}^{2} / n_{1} n_{2}\right)$. A hipótese nula é rejeitada para valores grandes de $W$. 
Mardia(1972) fornece alguns valores críticos para o teste quando $n \leq 14$ e $q=3$. Nos casos en que esta tabela não se aplica e as amostras forem pequenas, podemos adotar o procedimento de aleatorização descrito no item (a), que pode ser facilmente generalizado para $q$ amostras, $q \geq 2$ (ver Fisher(1993)). Se $n_{i} \geq 14, i=1, \ldots, q, W$ tem distribuição próxima a uma $\chi^{2}$ com $(2 q-2)$ graus de liberdade, sob $H_{0}$.

\subsection{Generalização para dados axias}

Como citamos na seção 2.7, quando os dados são axias, os ângulos observados estão todos distribuídos em um arco de comprimento $180^{\circ}$ do círculo de raio unitário. Para aplicarmos as técnicas descritas nas seções anteriores, precisamos relacionar o período de $180^{\circ}$ com o comprimento da circunferência toda. Assim, se $\theta_{1}, \ldots, \theta_{n}$ são observações de uma população unimodal em $\left(0, \frac{360^{\circ}}{2}\right)$ as observações transformadas:

$$
\theta_{i}^{\prime}=2 \theta_{i} \quad\left(\bmod 360^{\circ}\right), \quad i=1, \ldots, n
$$

vão pertencer a uma população unimodal em $\left(0^{\circ}, 360^{\circ}\right)$. Os testes descritos na seções anteriores podem ser aplicados às observações transformadas. Fisher(1993) recomenda que, após a aplicação dos testes às observações transformadas, para efeito de estimação, medidas amostrais de locação tenham seus valores devididos por 2 e as medidas amostrais de dispersão pemaneçam inalteradas. 


\section{Capítulo 6}

\section{Análise de duas ou mais amostras da distribuição de von Mises}

\subsection{Introdução}

Neste capítulo consideramos duas ou mais amostras independentes de dados provenientes de distribuições de von Mises, e comparamos suas direções médias e dispersões através de testes de hipótese. Se a suposição de que as populações são de von Mises é verdadeira, devemos esperar que os testes que vamos apresentar sejam mais poderosos do que os procedimentos não paramétricos descritos no capítulo 5 . Nas seções 6.2 e 6.3 apresentamos inferências sobre direções médias, e nas seções 6.4 e 6.5, sobre parâmetros de concentração.

Adotamos a mesma notação do capítulo 5 , considerando que as q amostras de tamanho $n_{i}$ seguem uma distribuição de von Mises, $V M\left(\mu_{i}, \kappa_{i}\right)$.

\subsection{Testes de igualdade de direções médias}

Nosso objetivo é testar as hipóteses:

$$
\begin{aligned}
& H_{0}: \mu_{1}=\ldots=\mu_{q}=\mu, \\
& H_{1}: \text { pelo menos uma igualdade não é válida. }
\end{aligned}
$$

Se a hipótese nula é correta, a direção da resultante em cada amostra deve ser próxima ao valor da direção média comum $\mu$. No caso das amostras serem agrupadas, 
então a resultante na amostra conjunta também deve ter direção próxima a $\mu$, e sua magnitude não deve ser muito menor do que a soma das magnitudes das resultantes em cada amostra (ver Figura 6.1).

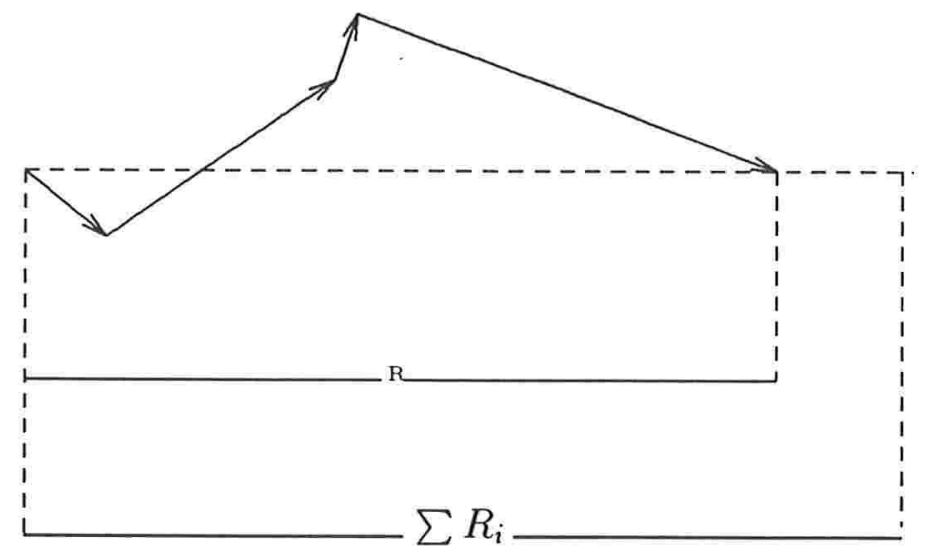

Figura 6.1: A base de um teste para uma direção média comum.

Uma estatística natural para testar a hipótese (6.1) é, então:

$$
A=R_{1}+R_{2}+\ldots+R_{q}-R
$$

e valores grandes de A sugerem que $H_{0}$ não é verdadeira. Os testes propostos para testar $H_{0}$ são em geral, funções de $\mathrm{A}$, e podem ser divididos em duas subclasses, dependendo dos $q$ parâmetros de concentração $\kappa_{1}, \ldots, \kappa_{q}$ serem ou não todos iguais.

\subsubsection{Os parâmetros de concentração não são todos iguais}

A conduta a ser adotada depende dos tamanhos das amostras e dos valores observados dos estimadores de máxima verossimilhança de $\kappa_{i}$.

a) Existe pelo menos um $n_{i}<25$ ou $\hat{\kappa}_{i}<2, i=1, \ldots, q$.

Usa-se método bootstrap, descrito na seção B.10, com reamostragem da distribuição de von Mises $\operatorname{VM}\left(0, \hat{\kappa}_{i}\right), i=1,2, \ldots, q$. A estatística a ser considerada é a mesma que a fornecida pela expressão(5.3), ou seja, 


$$
Y_{q}=2\left(\sum_{i=1}^{q} \frac{1}{\hat{\sigma}_{i}^{2}}-R_{M}\right)
$$

sendo agora:

$$
\begin{gathered}
\frac{1}{\hat{\sigma}_{i}^{2}}=n_{i} r_{i} \hat{\kappa}_{i} \\
R_{M}=\left(\hat{C}_{M}^{2}+\hat{S}_{M}^{2}\right)^{1 / 2}, \quad \hat{C}_{M}=\sum_{i=1}^{q} \cos \hat{\mu}_{i} / \hat{\sigma}_{i}^{2}, \quad \hat{S}_{M}=\sum_{i=1}^{q} \sin \hat{\mu}_{i} / \hat{\sigma}_{i}^{2}
\end{gathered}
$$

$\hat{\kappa}_{i}$ é o valor observado do estimador de máxima verossimilhança de $\kappa_{i}$,

$r_{i}$ é a resultante média na i-ésima amostra, e

$\hat{\delta}_{i}=\frac{1}{r_{i} \hat{\kappa}_{i}}$ é a dispersão amostral na i-ésima amostra, $i=1, \ldots, q$. $Y_{q}$.

A hipótese de igualdade das $q$ direções médias é rejeitada para valores grandes de

b) $n_{i} \geq 25, \hat{\kappa}_{i} \geq 2, i=1, \ldots, q$.

Neste caso, Watson(1983) mostrou que a distribuição de $Y_{q}$ é próxima de uma $\chi^{2}$ com $(q-1)$ graus de liberdade, isto é,

$$
Y_{q} \rightarrow \chi_{q-1}^{2}
$$

Rejeitamos a hipótese nula para valores grandes de $Y_{q}$.

\subsubsection{Os parâmetros de concentração são iguais}

Vários testes foram propostos para o caso em que $\kappa_{1}=\ldots=\kappa_{q}=\kappa$, desconhecido. Descreveremos inicialmente o teste de Watson- Willians (1956) adequado para comparar as direções médias de duas ou mais populações de von Mises quando o valor do parâmetro de concentração $\kappa$ é grande. Os testes propostos por Stephens(1972) e Mardia(1972), que podem ser aplicados a dados muito dispersos ( $\kappa$ pequeno) serão apresentados no final da seção. Como o valor de $\kappa$ é desconhecido, a decisão sobre qual procedimento deve ser adotado em um dado problema deve ser tomada com 
base em uma estimativa desse parâmetro. Uma possível estimativa pode ser obtida calculando-se o valor do estimador de máxima verossimilhança de $\kappa$ na amostra combinada, ou seja,

$$
\hat{\kappa}=A^{-1}(r)
$$

\section{a)Teste de Watson-Williams}

Watson e Willians(1956) mostraram que, quando $\kappa$ é grande,

$$
\begin{aligned}
U & =2 \kappa\left(\sum_{j=1}^{q} R_{j}-R\right) \rightarrow \chi_{q-1}^{2}, \quad \text { sob } H_{0} \\
V & =2 \kappa\left(n-\sum_{j=1}^{q} R_{j}\right) \rightarrow \chi_{n-q}^{2},
\end{aligned}
$$

e $U$ e $V$ são independentes. Os autores propuseram a seguinte estatística para o teste:

$$
F=\frac{(n-q)\left(\sum_{i=1}^{q} R_{i}-R\right)}{(q-1)\left(n-\sum_{i=1}^{q} R_{i}\right)}
$$

Para valores de $\kappa$ grandes, a distribuição de $F$, sob $H_{0}$, se aproxima de uma FSnedecor com $(q-1)$ e $(n-q)$ graus de liberdade. Rejeitamos $H_{0}$ se $F>F_{q-1, n-q, \alpha}$. Observemos que os cálculos necessários para a obtenção da estatística $F$ podem ser dispostos em um quadro de análise de variância, representado na Tabela (6.1).

Tabela 6.1: Tabela de análise de variância.

\begin{tabular}{|l|l|l|l|r|}
\hline fonte de variação & g.l & Soma de Quadrados & Quadrados médios & $\mathrm{F}$ \\
\hline entre amostras & $q-1$ & $\sum_{i=1}^{q} R_{i}-R$ & $\left(\sum_{i=1}^{q} R_{i}-R\right) /(q-1)=a$ & $a / b$ \\
dentro de amostras & $n-q$ & $n-\sum_{i=1}^{q} R_{i}$ & $\left(n-\sum_{i=1}^{q} R_{i}\right) /(n-q)=b$ & \\
\hline total & $n-1$ & $n-R$ &. & \\
\hline
\end{tabular}

Stephens(1972) propôs a seguinte modificação na estatística F:

$$
F^{*}=\left(1-\frac{3}{8 \kappa}\right) F
$$


e mostrou, através de simulação, que o fator de correção $g=1-\frac{3}{8 \kappa} \quad$ torna a distribuição de $F^{*}$ mais próxima da F-Snedecor com $(q-1)$ e $(n-q)$ graus de liberdade. $\mathrm{O}$ autor mostrou que, no caso de duas amostras, a aproximação é boa para $\kappa \geq 2$, e para mais de duas amostras, a aproximação é adequada para $\kappa \geq 1$. Nos casos em que a utilização de $F^{*}$ não é recomendada, podem ser adotados os procedimentos descritos a seguir. Observemos que, para $\kappa>10$, o fator de correção g é negligível, tornando $F$ e $F^{*}$ equivalentes.

\section{b)Teste exato de Stephens}

Stephens(1972) deduziu um teste exato para comparar as direções médias de duas populações de von Mises com iguais parâmetros de concentração. Esse teste se baseia na estatística $A$ dada em expressão (6.2). Os valores críticos para o teste são obtidos em tabelas especiais que não são particularmente fáceis de serem utilizadas. Upton e Fingleton(1989) sugeriram fórmulas aproximadas para a obtenção dos valores críticos do teste (ver Tabela 6.2). Essas fórmulas fornecem uma boa aproximação para o caso em que $n_{1}=n_{2}$, e são conservativas em outros casos.

Tabela 6.2: Fórmula para obter valores críticos para o teste de Stephens.

\begin{tabular}{|l|c|}
\hline nivel de significancia & Formula \\
\hline $5 \%$ & $n \cdot \exp \left(1,30-0,75 \ln (n)-3,785 R / n+14,0 R / n^{2}\right)$ \\
$1 \%$ & $n \cdot \exp \left(1,27-0,66 \ln (n)-3,30 R / n+12,4 R / n^{2}\right)$ \\
\hline
\end{tabular}

\section{c)Teste de Mardia}

Considerando o caso em que $0<\kappa<1$ e $q>2$, $(r<0,45) \operatorname{Mardia(1972)}$ mostrou que a estatística do teste da razão de verossimilhança pode ser escrita, de forma aproximada, como:

$$
-2 \ln \lambda \simeq \frac{2}{n}\left(1-\frac{\kappa^{2}}{8}+\frac{q}{2 n \kappa^{2}}\right)^{-1}\left(\left(\sum_{i=1}^{q} R_{i}\right)^{2}-R^{2}\right)=U
$$

Quando $H_{O}$ é verdadeira, $U$ tem distribuição próxima a uma $\chi_{q-1}^{2}$. Na prática, substituímos $\kappa$ por sua estimativa de máxima verossimilhança $\hat{\kappa}$, e rejeitamos $H_{0}$ para valores grandes de $U$. A aproximação acima é boa mesmo para valores pequenos de $n$, dado que $\kappa$ não esteja muito próximo de zero ou 1 . 


\subsection{Inferências após a comparação das direções médias}

Os testes descritos na seção anterior podem levar à rejeição ou não da hipótese de igualdade das direções médias. No caso de não rejeitarmos $H_{0}$, é interessante estimarmos a direção média comum $\mu$ por ponto e por intervalo. Por outro lado, se $H_{0}$ é rejeitada e tivermos três ou mais amostras, devemos prosseguir a análise com o objetivo de localizar as diferenças existentes. Procedimentos adequados a cada situação serão descritos a seguir.

\subsubsection{Estimação da direção média comum de duas ou mais distribuições de von Mises}

Para estimar a direção média comum $\mu$ devemos considerar se os parâmetros de concentração nas $q$ populações são ou não iguais.

a) $\kappa_{1}=\ldots=\kappa_{q}=\kappa$

Temos $q$ amostras de uma única população de von Mises. Logo, todos os dados podem ser combinados em uma única amostra de $n$ pontos e aplicar-se os métodos apresentados nas seções 4.4 .3 e 4.4.4.

b) $\kappa_{1}, \ldots, \kappa_{q}$ não são todos iguais

Adotamos o método descrito na seção 5.3 .2 para estimar $\mu$, considerando as expressões para os erros padrão circulares e dispersões circulares amostrais próprias à distribuição de von Mises, isto é:

$$
\hat{\sigma}_{i}=\frac{1}{\left(n_{i} r_{i} \hat{\kappa}_{i}\right)^{1 / 2}}, \quad \hat{\delta}_{i}=\frac{1}{\left(r_{i} \hat{\kappa}_{i}\right)^{1 / 2}}, \quad i=1, \ldots, q .
$$

Temos agora que:

$$
v_{i}=\left(r_{i} \hat{\sigma}_{i}^{2}\right)^{-1}, \quad v=\sum_{i=1}^{q} v_{i}, \quad w_{i}=\frac{v_{i}}{v} \quad \text { e } \quad \sigma_{M}^{2}=\sum_{i=1}^{q} \frac{w_{i}{ }^{2} r_{i}{ }^{2} \hat{\sigma}_{i}^{2}}{\left(\sum_{i=1}^{q} w_{i} r_{i}\right)^{2}} .
$$


A forma de construção do intervalo de confiança para a média comum $\mu$ depende dos tamanhos de amostras $n_{i}$ e das estimativas dos parâmetros de concentração $\hat{\kappa}_{i}$, $i=1, \ldots, q$.

$b_{1}$ ) pelo menos um $n_{i}<25$ ou $\hat{\kappa}_{i}<2$

Devem ser utilizadas as técnicas bootstrap descritas em $B .11$, com reamostragem a partir da distribuição von Mises $\operatorname{VM}\left(0, \hat{\kappa}_{i}\right), i=1, \ldots, q$, para estimar a direção média comum e construir um intervalo de confiança.

b) $n_{i} \geq 25, \hat{\kappa}_{i} \geq 2$

São utilizadas as expressões da seção 5.3.2 para amostras grandes. Neste caso, um estimador combinado por ponto para a direção média comum $\mu$ é dado por,

$$
\hat{\mu}_{w}= \begin{cases}\operatorname{arctag}\left(\frac{\bar{S}_{w}}{\bar{C}_{w}}\right) & \text { se } \bar{S}_{w}>0, \bar{C}_{w}>0 ; \\ 180^{0}+\operatorname{arctag}\left(\frac{\bar{S}_{w}}{\bar{C}_{w}}\right) & \text { se } \bar{C}_{w}<0 ; \\ 360^{0}+\operatorname{arctag}\left(\frac{\bar{S}_{w}}{\bar{C}_{w}}\right) & \text { se } \bar{S}_{w}<0, \bar{C}_{w}>0,\end{cases}
$$

onde $\bar{C}_{w}$ e $\bar{S}_{w}$ são dados pela expressão (5.5) utilizando-se para seu cálculo $w_{i}$ e $v_{i}$ como na expressão (6.10). Um intervalo de confiança com coeficiente de confiança $\gamma$ $=1-\alpha$ (Fisher, 1993) é dado por:

$$
\left[\hat{\mu}_{w} \pm \operatorname{arcsen}\left(z_{\alpha / 2} \hat{\sigma}_{M}\right)\right]
$$

onde

$\hat{\mu}_{w}$ é dado em expressão (6.11) e $\hat{\sigma}_{M}$ é obtido a partir de (6.10).

\subsubsection{Construção de intervalos de confiança para a diferença de duas direções médias}

No caso de termos três ou mais amostras de populações de von Mises com o mesmo parâmetro de concentração $\kappa$, podemos localizar as diferenças existentes entre as direções médias construindo intervalos de confiança para as diferenças;

$$
\delta_{i j}=\left(\mu_{i}-\mu_{j}\right) \quad(\bmod 2 \pi), i, j=1, \ldots, q, i>j .
$$


Mardia mostrou que $\bar{\theta}_{i} \mid R_{i}$ e $\bar{\theta}_{j} \mid R_{j}$, têm distribuição $V M\left(\mu_{i}, \kappa R_{i}\right)$ e $V M\left(\mu_{j}, \kappa R_{j}\right)$ respectivamente, e a diferença:

$$
d_{i j}=\left(\bar{\theta}_{i}-\bar{\theta}_{j}\right) \quad(\bmod 2 \pi)
$$

tem distribuição próxima a uma $V M\left(\delta_{i j}, \kappa_{i j}^{*}\right)$, onde $\kappa_{i j}^{*}$ é tal que:

$$
A\left(\kappa_{i j}^{*}\right)=A\left(\kappa R_{i}\right) \cdot A\left(\kappa R_{j}\right)
$$

Na prática, substituimos $\kappa$ por seu estimador de máxima verossimilhança $\hat{\kappa}$ dado por:

$$
A(\hat{\kappa})=\frac{R_{1}+\ldots+R_{q}}{n} .
$$

Assim, a distribuição de $d_{i j}$ pode ser aproximada por uma $V M\left(\delta_{i j}, \hat{\kappa}_{i j}^{*}\right)$, onde

$$
A\left(\hat{\kappa}_{i j}^{*}\right)=A\left(\hat{\kappa} R_{i}\right) \cdot A\left(\hat{\kappa} R_{j}\right) .
$$

Seja $\theta$ uma variável aleatória distribuída segundo uma $V M\left(0, \kappa_{i j}^{*}\right)$ e suponha que, para um dado $\alpha, \nu$ é tal que:

$$
P(\pi-\nu<\theta<\pi+\nu)=\alpha,
$$

onde o arco $(\pi-\nu, \pi+\nu$ ) inclui $\pi$ (ver Figura 6.2). Então um intervalo de confiança para $\delta_{i j}$ com coeficiente de confiança $\gamma=1-\alpha$ é dado por:

$$
\operatorname{arc}\left\{\left(d_{i j}+\pi-\nu\right)(\bmod 2 \pi) ;\left(d_{i j}+\pi+\nu\right) \quad(\bmod 2 \pi)\right\},
$$

onde o arco $\left(d_{i j}+\pi-\nu, d_{i j}+\pi+\nu\right)$ inclui $d_{i j}$. Suponhamos que estamos interessados em fazer c comparações das q médias duas a duas a um nível de significância, no máximo, igual a um valor $\alpha^{*}$ fixado. Devemos, então, na construção do intervalo (6.18), fixar $\alpha=\frac{\alpha^{*}}{c}$.

Alternativamente, considerando que $d_{i j} \sim V M\left(\delta_{i j}, \kappa_{i j}^{*}\right)$, onde $\kappa_{i j}^{*}$ satisfaz a expressão (6.14), podemos construir intervalos de confiança para as diferenças $\delta_{i j}, \quad i, j=$ $1, \ldots, q, \quad i>j$, utilizando os procedimentos de estimação da média de uma distribuição de von Mises apresentados na seção 4.4. Propomos que a escolha entre o método bootstrap e o assintótico seja feita de acordo com a Tabela 4.1, considerandose $\hat{\kappa}^{*}=\min \left(\hat{\kappa}_{i j}^{*}\right)$ no lugar de $\hat{\kappa}$ na primeira coluna, e $n^{*}=\min \left(n_{1}, \ldots, n_{q}\right)$ na terceira coluna da tabela.

Nas situações em que for adotado o método bootstrap, devemos utilizar o procedimento de reamostragem descrito em B.6. 
Se $\hat{\kappa}^{*}$ e $n^{*}$ forem tais que os resultados para grandes amostras são adequados, o intervalo de confiança para $\delta_{i j}$, com coeficiente de confiança $\gamma=1-\alpha$ é:

$$
\left(\bar{\theta}_{i}-\bar{\theta}_{j}\right) \pm \operatorname{arcsen}\left(z_{\frac{\alpha}{2}}\left(\hat{\kappa}_{i j}^{*} A\left(\hat{\kappa}_{i j}^{*}\right)\right)^{-\frac{1}{2}}\right)
$$

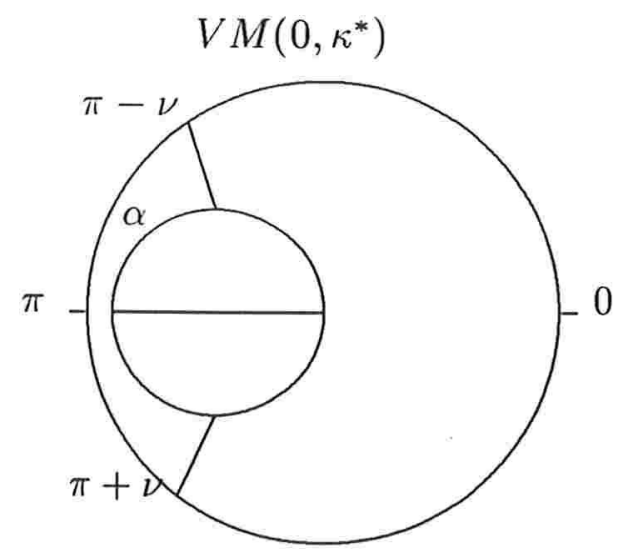

Figura 6.2: Representação do arco $(\pi-\nu, \pi+\nu)$ em uma $V M\left(0, \kappa^{*}\right)$

\subsection{Teste de igualdade de parâmetros de concen- tração de duas ou mais distribuições de von Mises}

Consideremos o problema de testar a hipótese:

$$
H_{0}: \kappa_{1}=\ldots=\kappa_{q}
$$

contra a alternativa de que pelo menos um $\kappa_{i}$ é diferente dos demaís. Sejam:

$$
\begin{aligned}
\bar{\theta}_{i} & =\hat{\mu}_{i} \text { a direção média na i-ésima amostra, } \\
d_{i j} & =\left|\operatorname{sen}\left(\theta_{i j}-\bar{\theta}_{i}\right)\right|, \quad j=1, \ldots, n_{i}, \\
\bar{d}_{i} & =\sum_{j=1}^{n_{i}} \frac{d_{i j}}{n_{i}}, \quad i=1, \ldots, q \mathrm{e}
\end{aligned}
$$




$$
\bar{d}=\sum_{i=1}^{q} \frac{n_{i} \bar{d}_{i}}{n}
$$

Observemos que $d_{i j}$ pode ser considerado como o "resíduo" da j-ésima observação no iésimo grupo. Se as concentrações nas $q$ populações forem iguais, devemos esperar que os valores médios dos "resíduos" $\bar{d}_{i}$ nas $q$ amostras sejam próximos a $\bar{d}$. Fisher(1986) sugeriu, então, a seguinte estatística para testar a hipótese (6.20):

$$
f_{q}=\frac{(n-q) \sum_{i=1}^{q}\left(\bar{d}_{i}-\bar{d}\right)^{2}}{(q-1) \sum_{i=1}^{q} \sum_{j=1}^{n_{i}}\left(d_{i j}-\bar{d}_{i}\right)^{2}} .
$$

Rejeitamos $H_{0}$ para valores grandes de $f_{q}$. O procedimento para se obter a região crítica do teste depende dos tamanhos das amostras e das dispersões amostrais. Em primeiro lugar devemos obter $\tilde{\kappa}$ a partir da expressão :

$$
\tilde{\kappa}=\operatorname{mediana}\left(\hat{\kappa}_{1}, \ldots, \hat{\kappa}_{q}\right)
$$

ou seja, $\tilde{\kappa}$ é a mediana das estimativas de $\kappa$ nas $q$ amostras. Então, podem ocorrer as seguintes situações:

a) $n_{i} \geq 10, \tilde{\kappa} \geq 1, . i=1, \ldots, q$.

$\mathrm{O}$ valor crítico do teste, a um nível de significância $\alpha$ é dado pelo quantil de ordem $(1-\alpha)$ da distribuição $F_{q-1, n-q}$.

\section{b) Ao menos uma das desigualdades em a) não está satisfeita}

Neste caso, devemos usar um teste aleatorizado (ver Fischer(1993) seção 8.5). Cada amostra deve ser centrada em sua média e procedemos ao teste aleatorizado baseado na alocação de $n$ valores centrados nas $q$ amostras, calculando a estatística $f_{q}$ em cada uma das alocações aleatorizadas.

Outros testes para a hipótese (6.20) foram propostos na literatura. Entretanto, segundo Fisher(1993), esses procedimentos são sensíveis a outliers e à suposição de que a população amostrada é von Mises. 


\subsection{Estimação do parâmetro de concentração co- mum de duas ou mais distribuições de von $\mathrm{Mi}$ -} ses

Suponhamos que a hipótese (6.20) não seja rejeitada. É interessante, então, estimarmos o parâmetro de concentração comum $\kappa$. Se pudermos assumir que as $q$ direções médias são iguais, podemos considerar a amostra conjunta de tamanho $n$ e aplicar a técnica apresentada na Subseção 4.4.3. Se não dispomos de informações sobre as direções médias, ou sabemos que elas são diferentes, o procedimento a ser adotado depende da magnitude de $\kappa$. O valor mediano $\tilde{\kappa}$ dado em (6.23) pode ser utilizado na escolha do procedimento a ser adotado:

a) $\tilde{\kappa}<2,0$

Seja

$$
\begin{aligned}
R_{t o t} & =R_{1}+\ldots+R_{q} \mathrm{e} \\
r_{\text {tot }} & =\frac{R_{\text {tot }}}{n} .
\end{aligned}
$$

Um estimador para $\kappa$ pode ser obtido a partir de expressão (4.19), utilizando-se $R_{\text {tot }}$ no lugar de $R$, isto é:

$$
\hat{\kappa}=\left\{\begin{array}{lll}
2 \bar{R}_{t o t}+\bar{R}_{t o t}^{3}+\frac{5 \bar{R}_{t o t}^{5}}{6} & \text { se } & \bar{R}_{t o t}<0,53 \\
-0,4+1,39 \bar{R}_{t o t}+\frac{0,43}{1-\bar{R}_{t o t}} & \text { se } & 0,53 \leq \bar{R}_{t o t}<0,85 \\
\bar{R}_{\text {tot }}^{3}-4 \bar{R}_{\text {tot }}^{2}+3 \bar{R}_{t o t} & \text { se } & \bar{R}_{t o t} \geq 0,85 .
\end{array}\right.
$$

Para a construção de um intervalo de confiança para $\kappa$ devemos adotar o método bootstrap (ver B.12).

b) $\tilde{\kappa} \geq 2,0$

A estimativa por ponto para $\kappa$ é obtida da mesma forma que em a). O intervalo de confiança para $\kappa$ é construído considerando-se os resultados assintóticos já utilizados na seção 4.4.4(b). Fixando um coeficiente de confiança $\gamma=1-\alpha$, os limites inferior e superior do intervalo são, então, dados respectivamente por:

$$
\kappa_{I}=\left[1+(1+3 a)^{1 / 2}\right] / 4 a
$$




$$
\kappa_{S}=\left[1+(1+3 b)^{1 / 2}\right] / 4 b
$$

onde:

$$
a=\left(n-R_{t o t}\right) / \chi_{n-q, 1-\frac{\alpha}{2}}^{2}
$$

e

$$
b=\left(n-R_{t o t}\right) / \chi_{n-q, \frac{\alpha}{2}}^{2}
$$

onde $\chi_{n-q, 1-\frac{\alpha}{2}}^{2}$ e $\chi_{n-q, \frac{\alpha}{2}}^{2}$ são, respectivamente os quantis de ordem $1-\frac{\alpha}{2}$ e $\frac{\alpha}{2}$ da distribuição $\chi^{2}$ com $n-q$ graus de liberdade. 


\section{Capítulo 7}

\section{Aplicações}

\subsection{Introdução}

Este capítulo tem como objetivo ilustrar a teoria exposta nos capítulos anteriores, e para tanto são apresentadas três aplicações numéricas.

$\mathrm{Na}$ aplicação 1 analisamos dados observados e gentilmente cedidos pelo Prof. Dr. Ronald Ranvaud, do Instituto de Ciências Biomédicas da Universidade de São Paulo.

Os dados utilizados nas aplicações 2 e 3 correspondem a amostras de distribuições de von Mises, geradas aleatoriamente pelo módulo GVON.SAS listado no Apêndice C.

Efetuamos cálculos com o auxílio de rotinas especiais que desenvolvemos no aplicativo SAS. Esses programas encontram-se no Apêndice C.

\subsection{Aplicação 1}

Os dados da Tabela 7.1 correspondem ao ângulo de desaparecimento de pombos, em graus, após terem sido libertados do cativeiro. A direção $0^{\circ}$ corresponde ao norte geográfico. O objetivo do pesquisador é verificar se a posição do sol afeta a direção escolhida pelos pombos nos períodos da manhã, meio-dia, e tarde.

$\mathrm{Na}$ Figura 7.1, representamos graficamente os dados observados, e os vetores 
Tabela 7.1: Ângulo de desaparecimento de pombos.

\begin{tabular}{||l|l||}
\hline manhã & $275 ; 285 ; 185 ; 290 ; 195 ; 225 ; 335 ; 285 ; 120 ; 30 ; 10 ; 85 ; 90 ; 80 ;$ \\
& $350 ; 60 ; 345 ; 35 ; 65 ; 250 ; 60 ; 175 ; 325 ; 330 ; 280 ; 350 ; 185 ; 20 ; 30$ \\
\hline \multirow{3}{*}{ meio-dia } & $310 ; 15 ; 295 ; 45 ; 330 ; 115 ; 235 ; 320 ; 40 ; 315 ; 15 ; 310 ; 20 ; 5 ;$ \\
& $340 ; 330 ; 20 ; 70 ; 320 ; 280 ; 225 ; 245 ; 255 ; 305 ; 295 ; 340 ; 45 ;$ \\
& $280 ; 115 ; 150 ; 325 ; 320 ; 50 ; 35 ; 65 ; 355 ; 270 ; 250 ; 300 ; 220$ \\
\hline tarde & $35 ; 70 ; 60: 65 ; 350 ; 330 ; 350 ; 185 ; 305 ; 55 ; 10 ; 45 ; 10 ;$ \\
& $50 ; 215 ; 320 ; 50 ; 110 ; 15 ; 60 ; 345 ; 325 ; 60 ; 355 ; 5$ \\
\hline
\end{tabular}

média nas três amostras.

Na Tabela 7.2 apresentamos algumas medidas de posição e dispersão amostrais.

Os resultados observados sugerem que os dados referentes a pombos soltos no período da manhã são mais dispersos que os dos libertados no período da tarde e ao meio-dia. Os pombos libertados ao meio-dia tendem a tomar a direção nordeste, enquanto que os soltos no período da tarde tendem a tomar o rumo noroeste.

Com o objetivo de verificar, se em cada grupo temos uma distribuição uniforme, aplicamos a cada amostra o teste de Rayleigh (ver seção 4.2).

$\mathrm{Na}$ amostra 1 não rejeitamos a hipótese de uniformidade $(\mathrm{p}=0,304)$, o que significa que pombos, no período da manhã, não apresentam uma direção de preferência. Para os grupos 2 e 3 a hipótese de uniformidade foi rejeitada $(\mathrm{p}<0,001)$, o que significa que os pombos soltos ao meio-dia e tarde apresentam uma direção preferencial. Prosseguimos então a análise considerando apenas os grupos 2 e 3 .

Para avaliar se os dados seguem uma distribuição de von Mises, consideramos a estatística $U^{2}$ dada pela expressão (4.10). A hipótese de que os dados seguem uma distribuição de von Mises foi rejeitada nos dois grupos $(p<0,05)$.

Adotamos, então, uma técnica não paramétrica para comparar as direções médias tomadas pelos pombos libertados ao meio-dia e à tarde (ver seção 5.3). O quociente entre as dispersões nos dois grupos é dado por: $2,096 / 0,893=2,347$. Portanto, as dispersões amostrais podem ser consideradas próximas e vamos comparar as direções médias adotando o método $P$.

$\mathrm{O}$ valor observado da estatística foi $Y_{2}=11,99$. Rejeitamos $H_{0}(p=0,0025)$ o que significa que as direções médias ao meio-dia e à tarde são diferentes. 


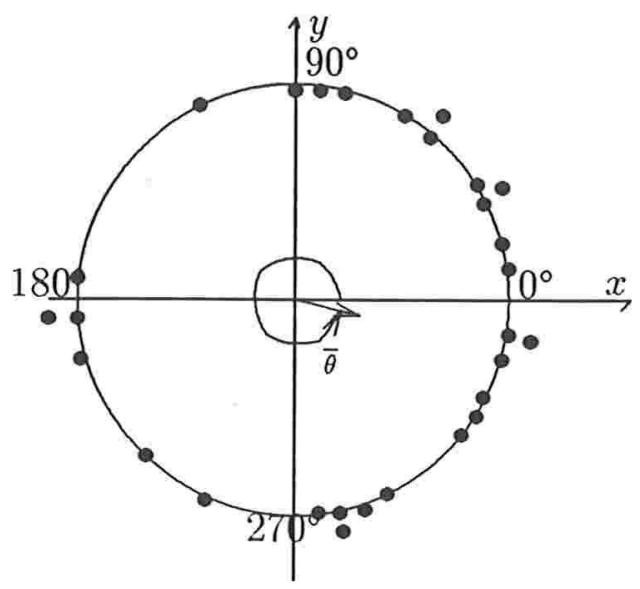

manhã

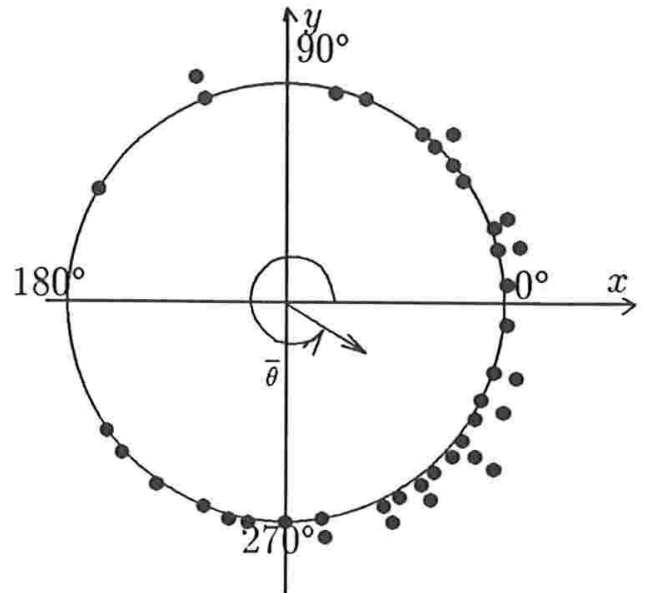

meio dia

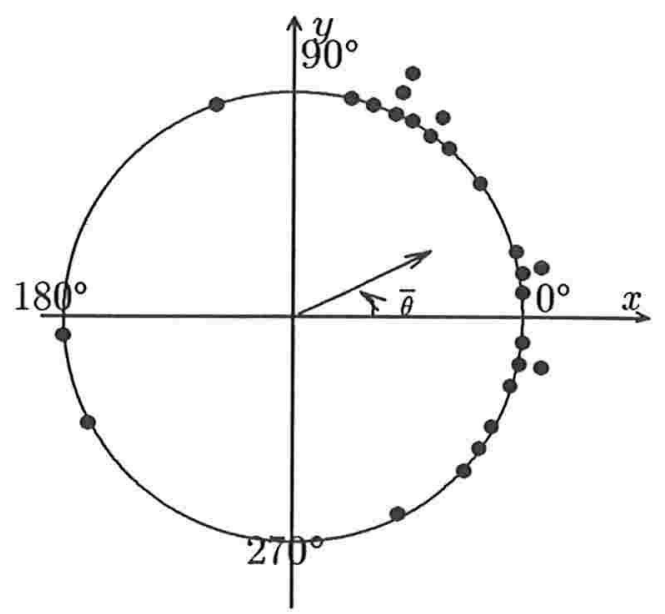

tarde

Figura 7.1: Representação gráfica do vetor média nos três grupos: manhã, meio-dia e tarde. 
Tabela 7.2: Medidas de posição e dispersão amostrais e tamanhos das amostras.

\begin{tabular}{|c|r|r|r|}
\hline \hline estatística & manhã & meio-dia & tarde \\
\hline $\bar{\theta}_{i}$ & 352,467 & 330,955 & 22,031 \\
$\tilde{\theta}_{i}$ & 345,000 & 325,000 & 15,000 \\
$r_{i}$ & 0,202 & 0,469 & 0,621 \\
$\hat{\delta}_{i}$ & 10,546 & 2,096 & 0,893 \\
$\hat{\sigma}_{i}$ & 0,603 & 0,229 & 0,189 \\
$n_{i}$ & 29 & 40 & 25 \\
\hline \hline
\end{tabular}

\subsection{Aplicação 2}

Com o objetivo de ilustrar a análise de uma amostra da distribuição de von Mises, consideremos os dados da Tabela 7.3, que correspondem a uma amostra da distribuição von Mises com parâmetros $\mu=45^{0}$ e $\kappa=1$ gerada utilizando-se o programa GVON.SAS.

Tabela 7.3: Amostra da distribuição de von Mises $\left(45^{0}, 1\right)$.

\begin{tabular}{|c||l|}
\hline \hline amostra & 28,$60 ; 191,83 ; 271,57 ; 43,11 ; 279,39 ;$ \\
& 51,$97 ; 80,90 ; 69,39 ; 18,47 ; 118,92$ \\
\hline \hline
\end{tabular}

Na tabela 7.4 apresentamos algumas estatísticas descritivas. Na figura 7.2 se observa a representação gráfica dos dados e o vetor média.

Suponhamos que os verdadeiros valores de $\mu$ e $\kappa$ sejam desconhecidos e que tenhamos interesse em testar $H_{0}: \mu=45^{\circ}$. Considerando a estatística $T_{n}$ dada em (4.31), observamos $T_{n}=0,1268$. Como temos $n<25$ e $\hat{\kappa}<2,0$, utilizamos o método bootstrap descrito em $B .7$, com $B=200$, para realizar o teste, obtendo, $T_{m_{\alpha}}=0,9125$. Desta forma não rejeitamos $H_{0}(\alpha=0,05)$.

Suponhamos também, que haja interesse em testar $H_{0}: \kappa=1$. Usamos também o método bootstrap descrito em $B .9(B=200)$, pelo qual calculamos um intervalo 
Tabela 7.4: Medidas de posição e dispersão amostrais.

\begin{tabular}{|c|r|}
\hline \hline Estatísticas & Amostra \\
\hline $\bar{\theta}$ & 50,008 \\
$\tilde{\theta}$ & 51,970 \\
$r$ & 0,375 \\
$\hat{\delta}$ & 2,177 \\
$\hat{\sigma}$ & 0,688 \\
$\hat{\kappa}$ & 0,562 \\
$n$ & 10 \\
\hline \hline
\end{tabular}

de confiança bootstrap com coeficiente de confiança $\gamma=0,95$, obtendo:

$$
\left(\hat{\kappa}_{(5)}^{*}, \hat{\kappa}_{(195)}^{*}\right)=(0,000 ; 8,435)
$$

Notemos que $\kappa=1$ pertence ao intervalo de confiança, e então não temos evidências para rejeitar a hipótese nula.

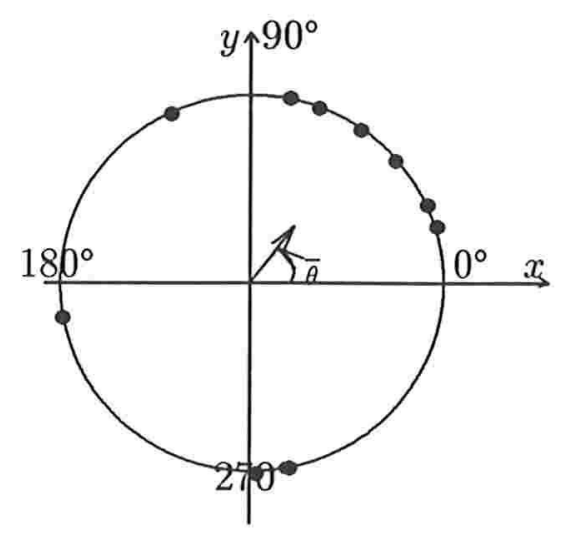

Figura 7.2: Representação gráfica dos dados da aplicação 2 e correspondente vetor média. 


\subsection{Aplicação 3}

Consideremos os dados da Tabela 7.5 que correspondem a amostras de distribuições von Mises: $V M\left(225^{0} ; 1,5\right), V M\left(130^{\circ} ; 1,5\right)$ e $V M\left(50^{\circ} ; 1,5\right)$

Tabela 7.5: Amostras de distribuições $V M(\mu, \kappa)$

\begin{tabular}{||l|l||}
\hline \multirow{3}{*}{ Amostra 1} & 40,$88 ; 66,18 ; 143,08 ; 154,24 ; 174,36 ; 176,75 ; 177,75 ; 178,49 ; 191,80 ; 191,82 ;$ \\
& 191,$83 ; 193,27 ; 245,74 ; 256,00 ; 256,24 ; 256,83 ; 258,73 ; 263,02 ; 269,15 ; 274,28 ;$ \\
& 276,$31 ; 278,59 ; 279,05 ; 280,75 ; 309,34 ; 312,49 ; 319,31 ; 322,44 ; 334,17 ; 359,46$ \\
\hline \multirow{3}{*}{ Amostra 2} & 26,$71 ; 31,21 ; 32,55 ; 49,13 ; 67,61 ; 73,48 ; 78,29 ; 81,05 ; 81,17 ; 85,07 ; 88,76 ;$ \\
& 91,$13 ; 98,45 ; 161,14 ; 161,25 ; 161,47 ; 161,66 ; 161,79 ; 161,82 ; 162,50 ; 161,81 ;$ \\
& 164,$83 ; 165,61 ; 168,47 ; 169,79 ; 177,21 ; 178,30 ; 183,28 ; 194,48 ; 275,39$ \\
\hline \multirow{3}{*}{ Amostra 3} & 0,$40 ; 0,86 ; 2,22 ; 4,79 ; 7,38 ; 7,90 ; 8,57 ; 8,82 ; 11,72 ; 12,09 ; 12,96 ;$ \\
& 13,$48 ; 14,03 ; 70,87 ; 76,36 ; 77,37 ; 80,35 ; 80,92 ; 81,36 ; 81,40 ; 82,75 ;$ \\
& 85,$00 ; 94,20 ; 99,76 ; 117,33 ; 130,19 ; 133,72 ; 137,25 ; 163,33 ; 206,39$ \\
\hline
\end{tabular}

Na Figura 7.3 representamos graficamente as amostras e seus vetores média. Na Tabela 7.6 apresentamos as estimativas de máxima verossimilhança dos parâmetros das distribuições de von Mises e algumas medidas de posição e dispersão.

Tabela 7.6: Medidas de posição e dispersão amostrais.

\begin{tabular}{|c|r|r|r|}
\hline \hline Estatísticas & Amostra 1 & Amostra 2 & Amostra 3 \\
\hline $\bar{\theta}_{i}$ & 251,064 & 129,718 & 57,694 \\
$\tilde{\theta}_{i}$ & 263,020 & 161,470 & 77,370 \\
$r_{i}$ & 0,457 & 0,574 & 0,613 \\
$\hat{\delta}_{i}$ & 1,458 & 1,113 & 1,022 \\
$\hat{\sigma}_{i}$ & 0,266 & 0,203 & 0,187 \\
$\hat{\kappa}_{i}$ & 1,027 & 1,408 & 1,561 \\
$n_{i}$ & 30 & 30 & 30 \\
\hline \hline
\end{tabular}

Suponhamos que os valores dos parâmetros sejam desconhecidos e que seja de interesse comparar os vetores médias das três populações.

Assumindo que as amostras foram retiradas de populações von Mises, testamos inicialmente $H_{0}: \kappa_{1}=\kappa_{2}=\kappa_{3}=\kappa$. Para isto utilizamos a estatística $f_{q}$ dada na expressão (6.22). $\mathrm{O}$ valor observado da estatística foi $f_{3}=0,147,(\mathrm{p}=0,864)$. Portanto, não se pode 
rejeitar, a um nível de significância fixado $\alpha=0.05$, a hipótese nula de que os parâmetros de concentração são iguais. Assim estimamos o parâmetro de concentração comum $\kappa$, obtendo $R_{\text {tot }}=0,548$ e $\hat{\kappa}=1,314$.

A seguir utilizamos a estatística de Watson-Williams dada na expressão (6.8), para testar se as direções média são iguais. Observamos que o valor da estatística é $F^{*}=47,90$. Rejeitamos $H_{0}(\mathrm{p}=1,255 \mathrm{E}-14)$, o que significa que as três direções médias populacionais não são iguais.

Com objetivo de localizar as diferenças existentes entre as médias, prosseguimos a análise construindo intervalos de confiança para as diferenças das médias duas a duas $(\gamma=0,95)$, adotando o procedimento descrito na subseção 6.3.2. Os intervalos obtidos são apresentados na Tabela 7.7. Concluimos que as direções médias são diferentes entre si.

Tabela 7.7: Intervalos de confiança para diferenças das direções médias $(\gamma=0,95)$

\begin{tabular}{|c|c|}
\hline \hline Diferenças & Intervalos de confiança \\
\hline$\mu_{1}-\mu_{2}$ & $(79,016 ; 163,675)$ \\
$\mu_{1}-\mu_{3}$ & $(153,877 ; 282,858)$ \\
$\mu_{2}-\mu_{3}$ & $(94,564 ; 156,840)$ \\
\hline
\end{tabular}




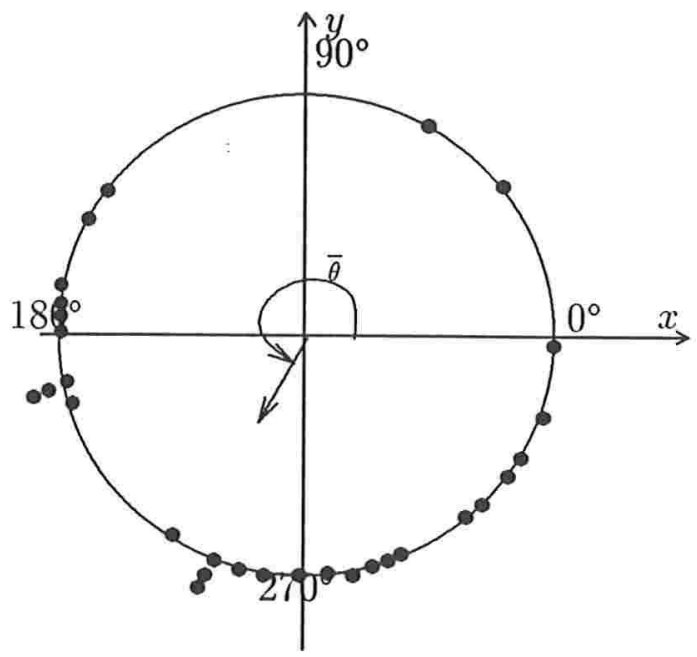

Amostra1

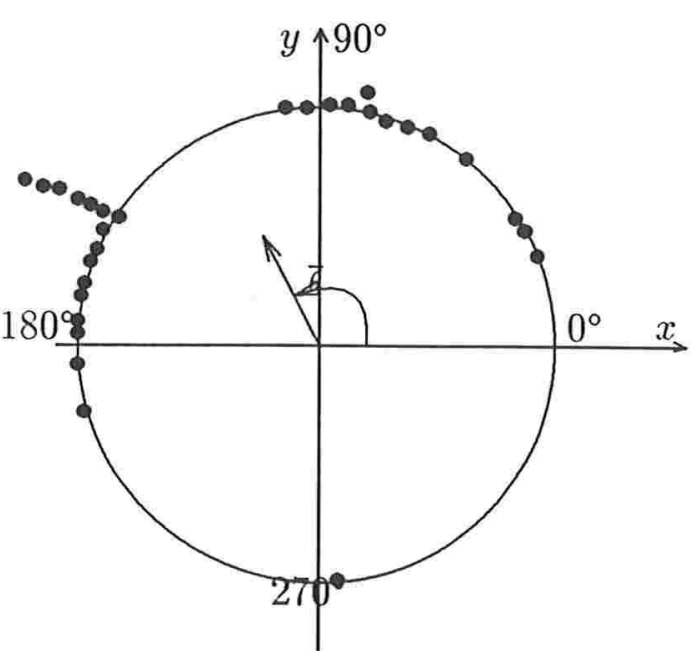

Amostra 2

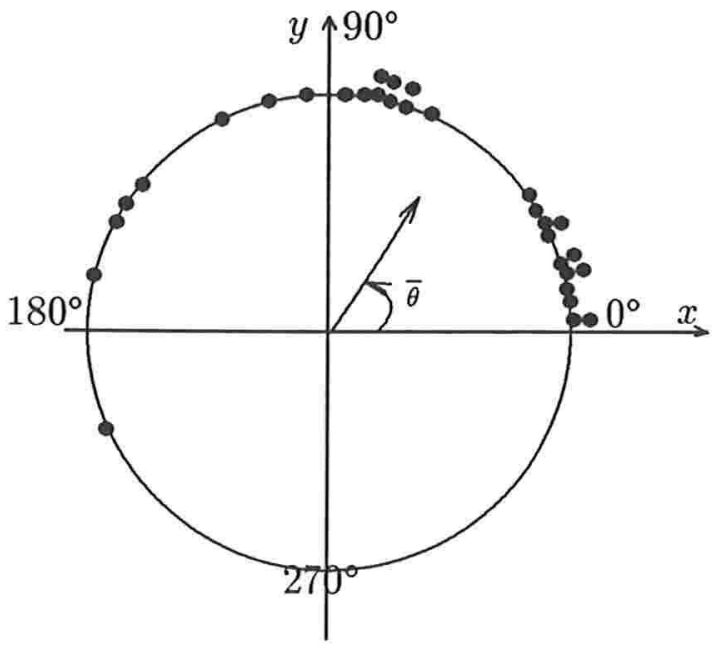

Amostra 3

Figura 7.3: Representação dos dados da aplicação 3 e seus vetores média. 


\section{Capítulo 8}

\section{Comentários finais}

Neste trabalho procuramos descrever de forma clara e correta a análise de uma amostra de dados circulares, bem como a estimação e comparação de medidas de dispersão e posição em experimentos completamente casualizados com um fator fixo.

A análise de experimentos envolvendo mais fatores, fatores aleatórios e seguindo outros planos experimentais merece ser estudada.

No que se refere aos planos experimentais completamente casualizados, experimentos fatorias podem ser analisados considerando-se os diferentes tratamentos como sendo os níveis de um único fator e aplicando-se as técnicas apresentadas neste trabalho. Alternativamente, métodos de análise que exploram a estrutura fatorial do experimento foram propostos por Anderson e Wu(1995), Harrison e Kanji(1988) e Harrison, Kanji e Gadsden(1986) e Underwood e Chapman(1985, 1989 e 1992). Experimentos nos quais os fatores se combinam de forma hierárquica foram considerados por Underwood e Chapman(1985).Problemas de análise de covariância podem ser resolvidos utilizando-se as técnicas de análise de regressão apresentadas em Fisher(1993).

A análise de experimentos seguindo um plano experimental em blocos completos casualizados é apresentada em Harrison e Kanji(1988) e Harrison, Kanji e Gadsden(1986).

Experimentos com medidas repetidas podem ser analisados segundo metodologia desenvolvida por Artes(1997). 
Não são de nosso conhecimento, até o momento, técnicas de análise de experimentos envolvendo fatores aleatórios.

Rotinas computacionais apropriadas à análise de dados circulares, além das apresentadas neste trabalho, também devem ser desenvolvidas. 


\section{Apêndice A}

\section{Demostração de propriedades do vetor média}

Propriedade 1: O vetor vetor média não depende da direção zero

Seja $\theta_{i}^{\prime}=\left(\theta_{i}-\alpha\right)$ o ângulo obtido de $\theta_{i}$ quando nova direção zero é $\alpha$. Temos então:

$$
\begin{aligned}
\bar{C}^{\prime} & =\frac{1}{n} \sum_{i=1}^{n} \cos \theta_{i}^{\prime}=\frac{1}{n} \sum_{i=1}^{n} \cos \left(\theta_{i}-\alpha\right) \\
& =\frac{1}{n} \sum_{i=1}^{n}\left(\cos \theta_{i} \cos \alpha+\operatorname{sen} \theta_{i} \operatorname{sen} \alpha\right) \\
& =\frac{1}{n}\left[\cos \alpha \sum_{i=1}^{n} \cos \theta_{i}+\operatorname{sen} \alpha \sum_{i=1}^{n} \operatorname{sen} \theta_{i}\right] \\
& =\cos \alpha \frac{1}{n} \sum_{i=1}^{n} \cos \theta_{i}+\operatorname{sen} \alpha \frac{1}{n} \sum_{i}^{n} \operatorname{sen} \theta_{i} \\
& =\cos \alpha \cdot \bar{C}+\operatorname{sen} \alpha \cdot \bar{S} .
\end{aligned}
$$

Considerando as seguintes equações:

$$
\cos \bar{\theta}=\frac{\bar{C}}{r}, \quad e \operatorname{sen} \bar{\theta}=\frac{\bar{S}}{r},
$$

obtemos:

$$
\begin{aligned}
\bar{C}^{\prime} & =r \cos \alpha \cos \bar{\theta}+r \operatorname{sen} \alpha \operatorname{sen} \bar{\theta} \\
& =r(\cos \alpha \cos \bar{\theta}+\operatorname{sen} \alpha \operatorname{sen} \bar{\theta}) \\
& =r \cos (\bar{\theta}-\alpha) .
\end{aligned}
$$


Temos também que:

$$
\begin{aligned}
\bar{S}^{\prime} & =\frac{1}{n} \sum_{i=1}^{n} \operatorname{sen} \theta_{i}^{\prime}=\frac{1}{n} \sum_{i=1}^{n} \operatorname{sen}\left(\theta_{i}-\alpha\right) \\
& =\frac{1}{n} \sum_{i=1}^{n}\left(\operatorname{sen} \theta_{i} \cos \alpha+\cos \theta_{i} \operatorname{sen} \alpha\right) \\
& =\cos \alpha \frac{1}{n} \sum_{i=1}^{n} \operatorname{sen} \theta_{i}+\operatorname{sen} \alpha \frac{1}{n} \sum_{i=1}^{n} \cos \theta_{i} \\
& =\cos \alpha \cdot \bar{S}+\operatorname{sen} \alpha \cdot \bar{C} . \\
& =\cos \alpha \cdot r \cdot \operatorname{sen} \bar{\theta}-\operatorname{sen} \alpha \cdot r \cdot \cos \bar{\theta} \\
& =r(\operatorname{sen} \bar{\theta} \cos \alpha-\cos \bar{\theta} \operatorname{sen} \alpha) \\
& =r \operatorname{sen}(\bar{\theta}-\alpha) .
\end{aligned}
$$

Fazendo $\bar{\theta}^{\prime}=(\bar{\theta}-\alpha) \quad(\bmod 2 \pi)$ temos:

$$
\bar{C}^{\prime}=r \cos \bar{\theta}^{\prime}, \quad \bar{S}^{\prime}=r \operatorname{sen} \bar{\theta}^{\prime} .
$$

$\log 0$

$$
r^{\prime}=\left(\bar{C}^{\prime}+\bar{S}^{\prime}\right)^{1 / 2}=r .
$$

Propriedade 2: $\sum_{i=1}^{n} \operatorname{sen}\left(\theta_{i}-\bar{\theta}\right)=0$.

Sabemos que,

$$
\begin{aligned}
\operatorname{sen}\left(\theta_{i}-\bar{\theta}\right) & =\operatorname{sen} \theta_{i} \cos \bar{\theta}-\cos \theta_{i} \operatorname{sen} \bar{\theta} \\
\sum_{i=1}^{n} \operatorname{sen}\left(\theta_{i}-\bar{\theta}\right) & =\sum_{i=1}^{n} \operatorname{sen} \theta_{i} \cos \bar{\theta}-\sum_{i=1}^{n} \cos \theta_{i} \operatorname{sen} \bar{\theta} \\
& =\cos \bar{\theta} \sum_{i=1}^{n} \operatorname{sen} \theta_{i}-\operatorname{sen} \bar{\theta} \sum_{i=1}^{n} \cos \theta_{i}
\end{aligned}
$$

Usando as equações (2.1) e (A.2) temos,

$$
\begin{aligned}
\sum_{i=1}^{n} \operatorname{sen}\left(\theta_{i}-\bar{\theta}\right) & =\frac{\bar{C}}{r} \cdot n \cdot \bar{S}-\frac{\bar{S}}{r} \cdot n \cdot \bar{C} \\
& =0 .
\end{aligned}
$$

Propriedade 3: $\sum_{i=1}^{n} \cos \left(\theta_{i}-\bar{\theta}\right)=n r$. 
Sabemos que:

$$
\begin{aligned}
\cos \left(\theta_{i}-\bar{\theta}\right) & =\cos \theta_{i} \cos \bar{\theta}+\operatorname{sen} \theta_{i} \operatorname{sen} \bar{\theta} \\
\sum_{i=1}^{n} \cos \left(\theta_{i}-\bar{\theta}\right) & =\sum_{i=1}^{n}\left(\cos \theta_{i} \cos \bar{\theta}+\operatorname{sen} \theta_{i} \operatorname{sen} \bar{\theta}\right) \\
& =\cos \bar{\theta} \sum_{i=1}^{n} \cos \theta_{i}+\operatorname{sen} \bar{\theta} \sum_{i=1}^{n} \operatorname{sen} \theta_{i}
\end{aligned}
$$

Usando as equações (2.1) e (A.2), temos:

$$
\begin{aligned}
\sum_{i=1}^{n} \cos \left(\theta_{i}-\bar{\theta}\right) & =\frac{\bar{C}}{r} \cdot n \cdot \bar{C}+\frac{\bar{S}}{r} \cdot n \cdot \bar{S} \\
& =\frac{n}{r}\left(\bar{C}^{2}+\bar{S}^{2}\right) \\
& =\frac{n}{r} r^{2} \\
& =n r .
\end{aligned}
$$

Propriedade 4: $\frac{1}{n} \sum_{i=1}^{n}\left(\theta_{i}-\bar{\theta}\right) \approx 2(1-r)$

Da propriedade 3 temos que:

$$
\frac{1}{n} \sum_{i=1}^{n} 2\left[1-\cos \left(\theta_{i}-\bar{\theta}\right)\right]=2[1-r] .
$$

Mas $2\left[1-\cos \left(\theta_{i}-\bar{\theta}\right)\right] \approx\left(\theta_{i}-\bar{\theta}\right)^{2}$, para $\left(\theta_{i}-\bar{\theta}\right)$ pequeno (ver Batschelet(1981)). Logo

$$
\frac{1}{n} \sum_{i=1}^{n}\left(\theta_{i}-\bar{\theta}\right)^{2} \approx 2(1-r),
$$

que é o análogo a $\frac{1}{n} \sum\left(x_{i}-\bar{x}\right)^{2}=S^{2}$ da estatística linear. 


\section{Apêndice B}

\section{Métodos Bootstrap}

\section{B.1 Introdução}

Os métodos bootstrap se referem a uma classe de procedimentos estatísticos computacionais que são frequentemente úteis na realização de testes de hipótese, ou na obtenção de medidas de variabilidade de estimadores por ponto, em situações nas quais procedimentos estatísticos analíticos não são válidos ou conhecidos.

Efron e Tibshirani(1986), descreveram a técnica bootstrap da seguinte forma: suponhamos que os dados observados

$$
\boldsymbol{\theta}=\left(\theta_{1}, \ldots, \theta_{n}\right)
$$

consistem em observações de uma variável aleatória $\Theta$ com função de distribuição desconhecida $F$ em $\Omega$, o espaço amostral comum das observações. Temos uma estatística de interesse, digamos $X(\theta)$, cuja distribuição é desconhecida. A partir de um processo de reamostragem das observações, e utilizando um método de Monte Carlo, podemos gerar a distribuição de $X(\theta)$ da seguinte forma:

- Passo 1:reamostragem. Usando um gerador de números aleatórios, retiramos, independentemente, uma amostra bootstrap, digamos:

$$
\theta_{1}^{*}
$$

- Passo 2:estimativa bootstrap. Na amostra bootstrap calculamos a estatística de interesse, obtendo:

$$
X\left(\theta_{1}^{*}\right)
$$


- Passo 3: repetição. Repetimos os passos 1 e 2 um grande número de vezes, digamos $B$ vezes, obtendo as amostras bootstrap:

$$
\theta_{1}^{*}, \ldots, \theta_{B}^{*} \quad \mathrm{e}
$$

os $B$ valores observados da estatística:

$$
X\left(\theta_{1}^{*}\right), \ldots, X\left(\theta_{B}^{*}\right) .
$$

- Passo 4: intervalo de confiança. Ordenamos as estatísticas bootstrap em ordem crescente e obtemos os percentis de acordo com o coeficiente de confiança desejado. A partir dos intervalos assim construídos podemos também realizar testes de hipótese.

Informações adicionais sobre os dados podem ser incorporadas ao procedimento, de forma a melhorá-lo.

Descrevemos nas próximas seções técnicas bootstrap que podem ser aplicadas a diferentes problemas envolvendo dados circulares.

\section{B.2 Intervalo de confiança para a direção média $\mu$}

Dada uma amostra aletória $\theta_{1}, \ldots, \theta_{n}$, gerar uma amostra bootstrap seguindo os seguintes passos:

- Passo 1: reamostragem. Gerar $n$ números aleatórios $u_{1}, \ldots, u_{n}$ a partir da distribuição uniforme $U[0,1]$, e calcular $i_{1}=$ parte inteira de $n u_{1}+1, \ldots, i_{n}=$ parte inteira de $n u_{n}+1$. A amostra booststrap é:

$$
\boldsymbol{\theta}_{1}^{*}=\left(\theta_{i_{1}}^{*}, \ldots, \theta_{i_{n}}^{*}\right)
$$

- Passo 2: calcular a estimativa booststrap $\hat{\mu}_{1}$ utilizando a expressão (2.2).

- Passo 3: repetir os passos 1 e 2 até obter um total de $B$ estimativas bootstrap da direção média; $\hat{\mu}_{1}, \ldots, \hat{\mu}_{B}$.

- Passo 4: construção do intervalo de confiança.

Obter

$$
\nu_{b}=\hat{\mu}_{b}-\bar{\theta}, \quad b=1, \ldots, B .
$$

onde $\bar{\theta}$ é a média na amostra original, e rearrajar os $\nu_{b}$ 's em ordem crescente, obtendo: $\nu_{(1)} \leq \ldots \leq \nu_{(B)}$.

Para $\alpha$ dado, achar $m=$ parte inteira de $\frac{B . \alpha}{2}$ e $l=B-m$. O intervalo de confiança é: $\left(\mu_{I}, \mu_{S}\right)$, onde $\mu_{I}=\nu_{(m+1)}+\bar{\theta}, \mu_{S}=\nu_{l}+\bar{\theta}$. 


\section{B.3 Teste de hipótese sobre a direção média $\mu$ em problemas com uma amostra}

Nosso objetivo é testar a hipótese $H_{0}: \mu=\mu_{0}$ (seção 4.3), onde $\mu_{0}$ é uma direção especificada. Na amostra original, calculamos:

$$
\psi_{1}=\theta_{1}-\left(\bar{\theta}-\mu_{0}\right), \ldots, \psi_{n}=\theta_{n}-\left(\bar{\theta}-\mu_{0}\right),
$$

onde $\bar{\theta}$ é a direção média amostral.

- Passo 1: reamostragem. Utilizar o passo 1 em B.2. Achar uma amostra booststrap considerando como amostra original $\psi_{1}, \ldots, \psi_{n}$.

- Passo 2: calcular a estatística $T=T\left(\theta_{1}^{*}, \ldots, \theta_{n}^{*}, \bar{\theta}\right)$ de $(4.8)$.

- Passo 3: repetir os passos 1 e 2 até obter um total de $B$ valores da estatística, isto é, $T_{1}, \ldots, T_{B}$.

- Passo 4: região crítica bilateral. Rearranjar os valores $\left|T_{1}\right|, \ldots,\left|T_{B}\right|$ em ordem crescente obtendo $T_{(1)} \leq, \ldots, \leq T_{(B)}$. Seja $\alpha$ o nível de significância do teste e $m_{\alpha}=$ parte inteira de $B(1-\alpha)+1$. Rejeita-se $H_{0}$, se $|T|>T_{\left(m_{\alpha}\right)}$, onde $T$ é calculado a partir da amostra original utilizando-se a expressão (4.8).

\section{B.4 Comparação das direções médias de duas ou mais populações}

Nosso objetivo é testar a hipótese

$$
H_{0}: \mu_{1}=, \ldots,=\mu_{q}=\mu
$$

a partir das $q$ amostras observadas:

$$
\theta_{i 1}, \ldots, \theta_{i n_{i}}, \quad i=1, \ldots, q
$$

Obter:

$$
\psi_{i 1}=\theta_{i 1}-\bar{\theta}_{i}, \ldots, \psi_{i n_{i}}=\theta_{i n_{i}}-\bar{\theta}_{i}, \quad i=1, \ldots, q,
$$

onde $\bar{\theta}_{i}$ é a direção média observada na $i$-ésima amostra. 
- Passo 1: a partir da cada amostra

$$
\psi_{i 1}, \ldots, \psi_{i n_{i}}, \quad i=1, \ldots, q
$$

gerar uma amostra bootstrap considerando o Passo 1 em B.2, obtendo

$$
\theta_{i}^{*}=\left(\theta_{i 1}^{*}, \ldots, \theta_{i n_{\mathrm{i}}}^{*}\right), \quad i=1, \ldots, q .
$$

- Passo 2: calcular a estatística $Y_{q}$ como em (5.3) ou (5.4).

- Passo 3: repetir os passos 1 e 2 até obter um total de $B$ valores da estatística, $Y_{1}^{*}, \ldots, Y_{B}^{*}$.

- Passo 4: rearranjar os valores $\left|Y_{1}^{*}\right|, \ldots,\left|Y_{B}^{*}\right|$ em ordem crescente obtendo $Y_{(1)}^{*}, \ldots, Y_{(B)}^{*}$. Seja $\alpha$ o nível de significância do teste e $m_{\alpha}=$ parte inteira de $B(1-\alpha)+1$. Rejeita-se $H_{0}$ se $\left|Y_{q}\right|>Y_{\left(m_{\alpha}\right)}^{*}$, onde $Y_{q}$ é o valor da estatística de interesse observada na amostra original, obtido a partir de (5.3) ou(5.4).

\section{B.5 Estimação da direção média comum de duas ou mais distribuições}

Temos $q \geq 2$ amostras de dados circulares:

$$
\theta_{i 1}, \ldots, \theta_{i n_{i}}, \quad i=1, \ldots, q .
$$

de distribuições unimodais com a mesma direção média $\mu$. A forma de se combinar as amostras depende dos tamanhos das amostras e das dispersões amostrais serem ou não da mesma magnitude, conforme vimos na seção 5.3. Adotando o método adequado, calculamos inicialmente $\hat{\mu}$ da forma descrita nessa seção. Para construir o intervalo de confiança para $\mu$, com coeficiente de confiança $\gamma=1-\alpha$, adotamos o seguinte procedimento:

- Passo 1: reamostrar cada amostra obtendo:

$$
\boldsymbol{\theta}_{i}^{*}, \quad i=1, \ldots, q .
$$

- Passo 2: calcular a estimativa da direção média comum $\mu$, digamos $\hat{\mu}_{1}^{*}$ adotando o mesmo método considerado para o cálculo de $\hat{\mu}$.

- Passo 3: repetir os passos 1 e 2 até obter um total de $B$ estimativas da direção média,

$$
\hat{\mu}_{1}^{*}, \ldots, \hat{\mu}_{B}^{*} .
$$


- Passo 4: obter

$$
\nu_{b}=\hat{\mu}_{b}^{*}-\hat{\mu}, \quad b=1, \ldots, B . \mathrm{e},
$$

rearrajar os $\nu_{b}$ 's em ordem crescente, obtendo; $\nu_{(1)} \leq \ldots \leq \nu_{(B)}$.

Para $\alpha$ dado, achar $m=$ parte inteira de $\frac{B \alpha}{2}$. O intervalo de confiança é: $\left(\mu_{I}, \mu_{S}\right)$, onde

$$
\mu_{I}=\nu_{(m+1)}+\hat{\mu}, \quad \mu_{S}=\nu_{(l)}+\hat{\mu} \quad, \text { e } \quad l=B-m
$$

\section{B.6 Intervalo de confiança para a direção média $\mu$ de uma $V M(\mu, \kappa)$}

Dadas as estimativas $\hat{\mu}(2.2), \hat{\kappa}$ (4.19) obtidas da amostra original, proceder da seguinte forma:

- Passo 1: reamostragem. Simular $n$ valores $\theta_{1}^{*}, \ldots, \theta_{n}^{*}$ apartir de uma $V M(\hat{\mu}, \hat{\kappa})$, usando o programa ESTVON.SAS do Apêndice C

Os demais passos são análogos aos passos 2,3 e 4 descritos em B.2.

\section{B.7 Teste de hipótese sobre a direção média de uma população $V M(\mu, \kappa)$}

Considerando o processo de reamostragem descrito em B. 6 procedemos como em B. 3 considerando agora a estatística $T_{n}$ dada em (4.32).

\section{B.8 Intervalo de confiança para o parâmetro de concentração em uma $V M(\mu, \kappa)$}

Para determinar um intervalo de confiança para $\kappa$, considerar o processo de reamostragem descrito em B.6 para a construção de $B$ amostras bootstrap. Em cada amostra obter $\hat{\kappa}_{i}^{*}, i=1, \ldots, B$, que ordenados em forma crescente produzem:

$$
\hat{\kappa}_{(1)}^{*} \leq \cdots \leq \hat{\kappa}_{(B)}^{*} .
$$

Um intervalo de confiança para $\kappa$ com um coeficiente de confiança $\gamma=(1-\alpha)$ é: $\left(\hat{\kappa}_{(l+1)}^{*}, \hat{\kappa}_{(m)}^{*}\right)$, onde $l=$ parte inteira de $(B \alpha / 2+1 / 2)$ e $m=B-l$. 


\section{B.9 Teste de hipótese sobre a o parâmetro de con- centração em uma $V M(\mu, \kappa)$}

Nosso objetivo é testar $H_{0}: \kappa=\kappa_{0}$. Este teste é realizado considerando o intervalo de confiança bootstrap em B.8. Rejeita-se $H_{0}$ se $\kappa_{0}$ não pertence ao intervalo $\left(\hat{\kappa}_{(l+1)}^{*}, \hat{\kappa}_{(m)}^{*}\right)$.

\section{B.10 Teste de igualdade de direções médias de duas ou mais distribuições von Mises}

Temos $q$ amostras:

$$
\theta_{i 1}, \ldots, \theta_{i n_{i}}, \quad i=1, \ldots, q
$$

da distribuição von Mises. Em cada amostra calculamos $\hat{\mu}_{i}, \hat{\kappa}_{i}, \quad i=1, \ldots, q$.

- Passo 1: reamostragem. Simular uma amostra $\psi_{i 1}, \ldots, \psi_{i n_{i}}$ de tamanho $n_{i}$ a partir de uma $V M\left(0, \hat{\kappa}_{i}\right), i=1, \ldots q$ e obter:

$$
\theta_{i 1}^{*}=\psi_{i 1}+\hat{\mu}_{i}, \ldots, \theta_{i n_{i}}^{*}=\psi_{i n_{i}}+\hat{\mu}_{i}, \quad i=1, \ldots, q
$$

- Passo 2:estatística bootstrap. Calcular o valor da estatística $Y_{q}$ dada expressão (6.3).

Os passos seguintes são análogos aos passos 3 e 4 em B.4.

\section{B.11 Estimação da direção média comun de 2 ou mais distribuições von Mises}

- Passo 1: reamostragem. A partir de cada amostra $\theta_{i 1}, \ldots, \theta_{i n_{i}}, i=1, \ldots, q$, construir uma amostra $\left(\theta_{i 1}^{*}, \ldots, \theta_{i n_{i}}^{*}\right), i=1, \ldots, q$ da mesma forma que no passo 1 de B.10.

- Passo 2: estimativa bootstrap. Calcular a estimativa bootstrap $\hat{\mu}_{w}^{*}$, para este conjunto de $q$ amostras, utilizando a expressão (6.11).

Os passos seguintes são análogos aos passos 3 e 4 em $B .5$. 


\section{B.12 Estimação do parâmetro de concentração co- mum de duas ou mais distribuições de von Mises}

- Passo 1: reamostragem. A partir das amostras originais, construimos amostras bootstrap da mesma forma que no passo 1 de B.10.

- Passo 2: estimador bootstrap de $\kappa$. Calcular o estimador $\hat{\kappa}_{1}^{*}$ para este conjunto de q amostras na mesma maneira que $\hat{\kappa}$ foi calculado na expressão (6.25).

- Passo 3: repetir os passos 1 e $2 B$ vezes, obtendo $\kappa_{1}^{*}, \ldots, \kappa_{B}^{*}$, que ordenados produzem $\kappa_{(1)}^{*} \leq, \ldots, \leq \kappa_{(B)}^{*}$. Um intervalo dde confiança para $\kappa$ com um coeficiente confiança $\gamma=1-\alpha$ é:

$$
\left(\hat{\kappa}_{(l+1)}^{*}, \hat{\kappa}_{(m)}^{*}\right) \text {, }
$$

onde $l=$ parte inteira de $\left(B \frac{\alpha}{2}+\frac{1}{2}\right)$ e $m=B-l$. 


\section{Apêndice C}

\section{Programas}

Foram desenvolvidos programas com o auxilio do módulo IML do pacote estatístico SAS. Para a análise gráfica foi desenvolvido um programa no aplicativo S-PLUS.

Mais especificamente, temos 15 programas:

GRAF.TXT Faz gráficos de dados circulares.

LOC.SAS Calcula direção média e medidas de dispersão de dados circulares.

MEDNA.SAS Calcula a mediana de dados circulares.

AJUST.SAS Calcula a estatística para o teste de ajuste a uma distribuição de von Mises.

STEST.SAS Calcula a estatística do teste para $H_{0}: \mu=\mu_{0}$ e o intervalo de confiança para $\mu$ (n grande).

BOOTS.SAS Gera amostras bootstrap e calcula $\mu$ para cada amostra bootstrap.

INTBOOS.SAS Calcula intervalos de confiança bootstrap para a direção média em uma amostra.

PRINC2.SAS Teste de hipótese $H_{0}: \mu=\mu_{0}$ em uma amostra bootstrap

ESTVON.SAS Calcula os estimadores de máxima verossimilhaça dos parâmetros $\mu$ e $\kappa$ da distribuição de von Mises em cada amostra.

BOOTSP.SAS Gera amostras bootstrap para a distribuição de von Mises e estima $\mu$ e $\kappa$ para cada amostra.

PRINC1.SAS Testa a hipótese $H_{0}: \mu=\mu_{0}$ em uma amostra da distribuição de von Mises através do método bootstrap.

INTBOOP.SAS Calcula o intervalo de confiança para $\kappa$ e testa $H_{0}: \kappa=\kappa_{0}$ usando o método bootstrap 
TESMP.SAS Calcula a estatística para testar $H_{0}: \mu_{1}=\mu_{2}$ por procedimento não paramétrico.

TPCONVM.SAS Compara parâmetros de concentração de distribuições de von Mises ( $\mathrm{n}$ grande).

TEM2VM.SAS Compara direções médias de distribuições de von Mises.

GVON.SAS Gera amostras de tamanho $n$ da distribução de von Mises com parâmetros $\mu$ e $\kappa$ 


\section{Programa GRAF.TXT}

Módulo para construir gráficos de dados circulares no aplicativo S-Plus.

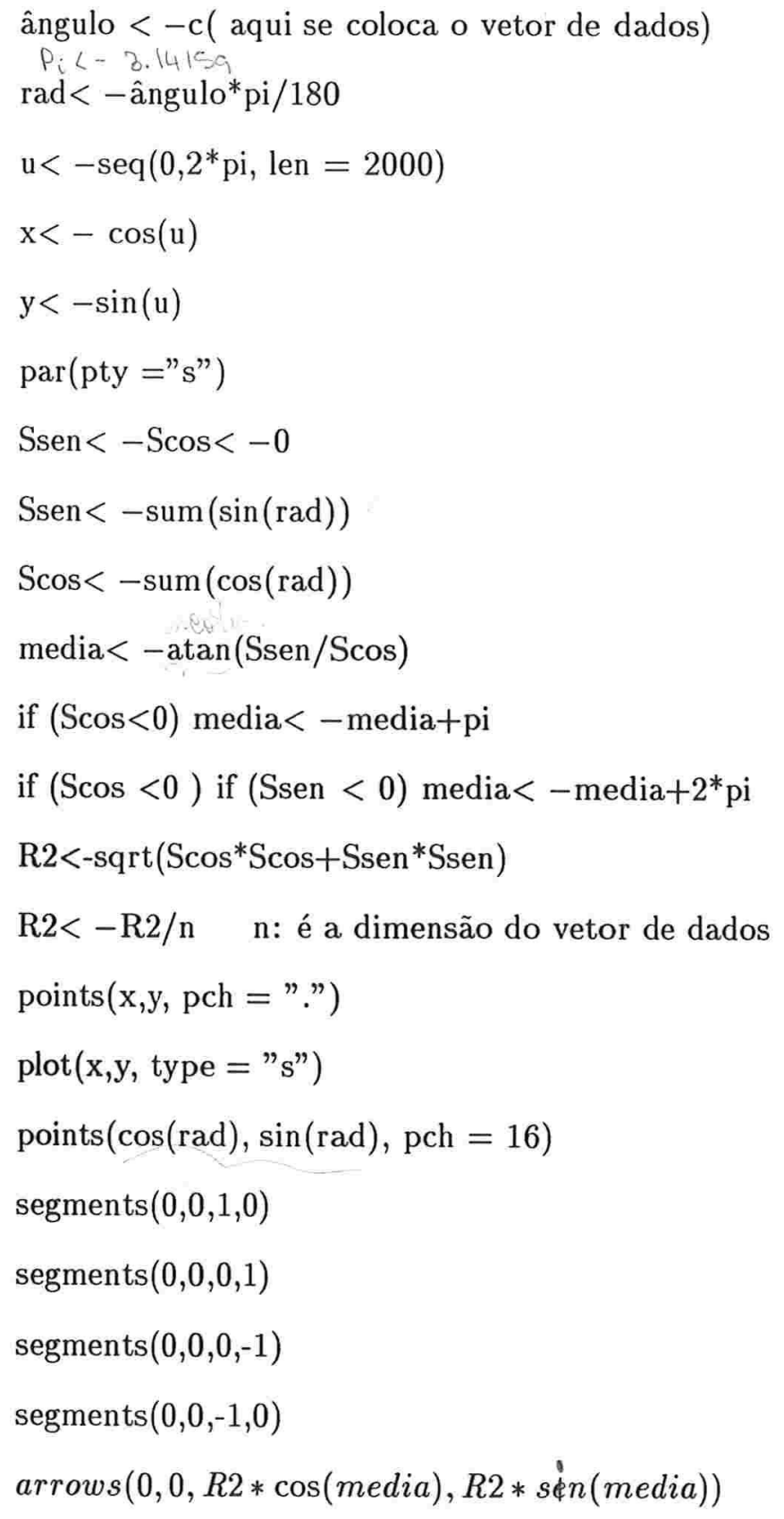




\section{Programa LOC.SAS}

Módulo para calcular as medidas descritivas em uma amostra.

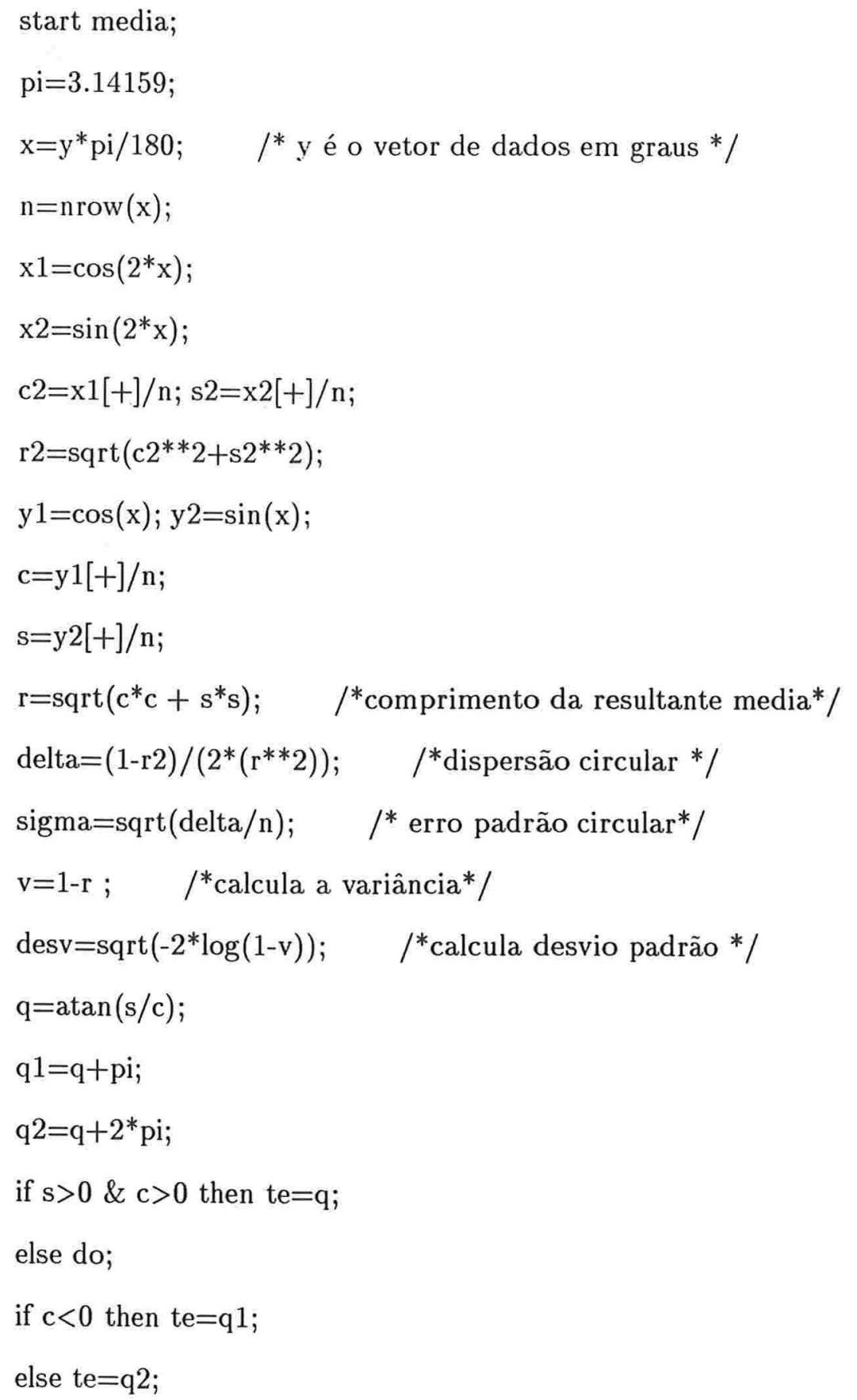


end;

media $=$ te*180/pi; /*direção média* /

print te media $\mathrm{v}$ desv delta sigma $\mathrm{r}$;

finish;

\section{Programa MEDNA.SAS}

Módulo para o cálculo da mediana amostral em uma amostra.

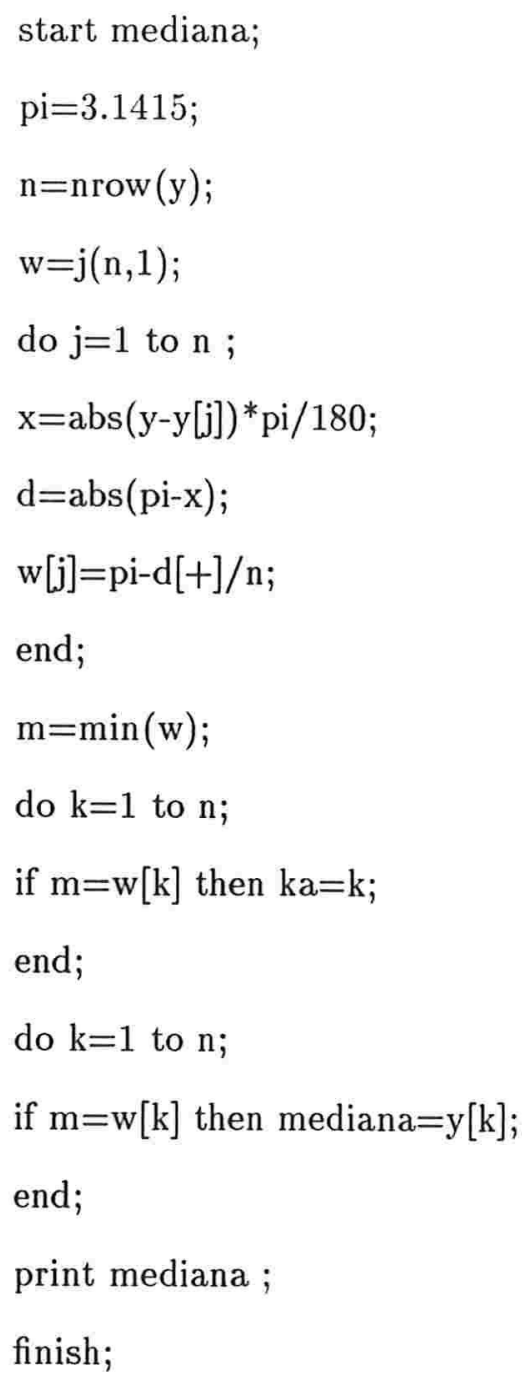




\section{Programa AJUST.SAS}

Módulo que calcula a estatística para o teste de aderência à distribuição de von Mises. start vajuste;

$\mathrm{n}=$ nrow $(\mathrm{y}) ; \quad \quad / *$ vetor da inversa da função de distribuição */

$\mathrm{z}=\mathrm{j}(\mathrm{n}, 1) ; \mathrm{zz}=\mathrm{z} ;$

$\mathrm{xbar}=\mathrm{y}[+] / \mathrm{n}$;

do $\mathrm{i}=1$ to $\mathrm{n}$;

$\mathrm{z}[\mathrm{i}]=\mathrm{y}[\mathrm{i}]^{* *} 2$

$\mathrm{zz}[\mathrm{i}]=\mathrm{y}[\mathrm{i}] *(2 * \mathrm{i}-1) / \mathrm{n}$;

end;

$\mathrm{u}=\mathrm{z}[+]-\mathrm{zz}[+]+\mathrm{n}^{*}\left(1 / 3-(\mathrm{xbar}-0.5)^{* *} 2\right)$;

print 'O valor da estatística é:' u ;

finish;

\section{Programa STEST.SAS}

Módulo para calcular a estatística para o teste da hipótese $H_{0}: \mu=\mu_{0}$ e intervalos de confiança para a direção média (n grande).

start steste ;

$\mathrm{pi}=3.14159$;

run media;

steste $=\operatorname{abs}(\sin ($ te-mu00)/sigma $) ; \quad / *$ estatística $\mathrm{T}$ para o teste $* /$

pvalue $=1$-probnorm (steste)

lic $=$ mu $0-\operatorname{arsin}\left(1.9604^{*}\right.$ sigma $) ; /{ }^{*}$ Intervalo de confiança com $95 \%$ de confiança* $/$

lis $=\mathrm{mu} 0+\operatorname{arsin}(1.9604 * \operatorname{sigma})$;

$\operatorname{licg}=\operatorname{lic}^{*} 180 / \mathrm{pi}$

lisg $=$ lis* $180 / \mathrm{pi}$;

$\operatorname{mug}=\mathrm{te}^{*} 180 / \mathrm{pi}$ 
print mug licg lisg steste pvalue ;

finish;

\section{Programa BOOTS.SAS}

Módulo para gerar amostras bootstrap, e estimar a direcão média em amostra bootstrap. libname novo'c:';

proc iml;

start boost;

$\mathrm{pi}=3.14159$;

$\mathrm{n}=\operatorname{nrow}(\mathrm{x}) ; \quad /{ }^{*} \mathrm{x}$ é o vetor de dados da amostra */

$\mathrm{c} 0=\mathrm{j}(\mathrm{n}, 1) ; \mathrm{s} 0=\mathrm{c} 0$;

$\mathrm{x} 01=\mathrm{c} 0 ; \mathrm{x} 02=\mathrm{c} 0$;

$\mathrm{x} 012=\mathrm{c} 0$;

do $\mathrm{j}=1$ to $n$;

$\operatorname{co}[\mathrm{j}]=\cos (\mathrm{x}[\mathrm{j}]) ; \mathrm{s} 0[\mathrm{j}]=\sin (\mathrm{x}[\mathrm{j}]) ;$

end;

$\mathrm{z} 01=\mathrm{c} 0[+] / \mathrm{n} ; \mathrm{z} 02=\mathrm{s} 0[+] / \mathrm{n} ;$

$\mathrm{q} 0=\operatorname{atan}(\mathrm{z} 02 / \mathrm{z} 01) ;$

$\mathrm{q} 01=\mathrm{q} 0+\mathrm{pi} ;$

$\mathrm{q} 02=\mathrm{q} 0+2 * \mathrm{pi} ;$

if $\mathrm{z} 02>0$ \& $\mathrm{z} 01>0$ then $\mathrm{mu} 0=\mathrm{q} 0 ; \quad / *$ mu0 é a direção média do vetor $\mathrm{x} * /$

else

if $\mathrm{z} 02<0 \& \mathrm{z} 01>0$ then $\mathrm{mu} 0=\mathrm{q} 02$;

else $\mathrm{mu} 0=\mathrm{q} 01$;

do $\mathrm{k}=1$ to $\mathrm{n}$;

$\mathrm{x} 01[\mathrm{k}]=(\mathrm{c} 0[\mathrm{k}]-\mathrm{z} 01) \# \# 2$;

$\mathrm{x} 02[\mathrm{k}]=(\mathrm{s} 0[\mathrm{k}]-\mathrm{z} 02) \# \# 2$; 
$\mathrm{x} 012[\mathrm{k}]=(\mathrm{c} 0[\mathrm{k}]-\mathrm{z} 01) \#(\mathrm{~s} 0[\mathrm{k}]-\mathrm{z} 02)$;

end;

$\mathrm{u} 01=\mathrm{x} 01[+] / \mathrm{n} ; \mathrm{u} 02=\mathrm{x} 02[+] / \mathrm{n} ; \mathrm{u} 012=\mathrm{x} 012[+] / \mathrm{n} ;$

$\mathrm{b} 0=(\mathrm{u} 01-\mathrm{u} 02) /\left(2^{*} \mathrm{u} 012\right)-\left((\mathrm{u} 01-\mathrm{u} 02)^{* *} 2 /\left(4^{*}\left(\mathrm{u} 012^{* *} 2\right)\right)+1\right)^{* *} 0.5 ;$

$\mathrm{t} 01=(\mathrm{u} 01 *(\mathrm{~b} 0 * * 2)+2 * \mathrm{~b} 0 * \mathrm{u} 012+\mathrm{u} 02)^{* *} 0.5 /(1+\mathrm{b} 0 * * 2) * * 0.5$;

$\mathrm{t} 02=(\mathrm{u} 01-2 * \mathrm{~b} 0 * \mathrm{u} 012+\mathrm{u} 02 *(\mathrm{~b} 0 * * 2)) /(1+\mathrm{b} 0 * * 2) * * 0.5 ;$

$\mathrm{v} 01=(\mathrm{t} 01 *(\mathrm{~b} 0 * * 2)+\mathrm{t} 02) /(1+\mathrm{b} 0 * * 2) ;$

$\mathrm{v} 02=(\mathrm{t} 01+\mathrm{t} 02 *(\mathrm{~b} 0 * * 2)) /(1+\mathrm{b} 0 * * 2) ;$

$\mathrm{v} 012=\mathrm{b} 0 *(\mathrm{t} 01-\mathrm{t} 02) /(1+\mathrm{b} 0 * * 2)$;

/*álculo amostras boostrap de tamano $\mathrm{n} 1 * /$

$\mathrm{cb}=\mathrm{j}(\mathrm{n} 1,1) ; \mathrm{sb}=\mathrm{j}(\mathrm{n} 1,1) ; \mathrm{q}=\mathrm{j}(\mathrm{n} 1,1) ;$

$\mathrm{q} 1=\mathrm{j}(\mathrm{n} 1,1) ; \mathrm{q} 2=\mathrm{j}(\mathrm{n} 1,1) ;$ muboos=j(n1,1);

$\operatorname{cbg}=\mathrm{j}(\mathrm{n} 1,1) ; \operatorname{sbg}=\mathrm{j}(\mathrm{n} 1,1) ; \mathrm{rbg}=\mathrm{j}(\mathrm{n} 1,1) ;$

$x 1=j(n, n 1) ; x 2=j(n, n 1) ; x 3=j(n, n 1) ;$

$\mathrm{um}=\mathrm{j}(\mathrm{n}, \mathrm{n} 1) ; \mathrm{c}=\mathrm{j}(\mathrm{n}, \mathrm{n} 1) ; \mathrm{s}=\mathrm{j}(\mathrm{n}, \mathrm{n} 1)$;

$\mathrm{z} 1=\mathrm{j}(\mathrm{n} 1,1) ; \mathrm{z} 2=\mathrm{j}(\mathrm{n} 1,1) ;$

$c 2=j(n, n 1) ; s c=j(n, n 1)$;

$\mathrm{s} 2=\mathrm{j}(\mathrm{n}, \mathrm{n} 1) ; \mathrm{u} 1=\mathrm{j}(\mathrm{n} 1,1) ; \mathrm{u} 2=\mathrm{j}(\mathrm{n} 1,1) ; \mathrm{u} 12=\mathrm{j}(\mathrm{n} 1,1)$;

$\mathrm{b}=\mathrm{u} 1 ; \mathrm{t} 1=\mathrm{u} 1 ; \mathrm{t} 2=\mathrm{u} 1 ; \mathrm{w} 1=\mathrm{u} 1 ; \mathrm{w} 2=\mathrm{u} 1 ; \mathrm{w} 12=\mathrm{u} 1 ; \mathrm{ml} 1=\mathrm{u} 1 ;$

$\mathrm{ml} 2=\mathrm{u} 1 ; \mathrm{ml} 3=\mathrm{u} 1 ; \mathrm{kml}=\mathrm{u} 1 ; \mathrm{kk} 1=\mathrm{u} 1 ;$

$\mathrm{kk} 2=\mathrm{u} 2 ;$ kest $=\mathrm{u} 1 ; \mathrm{kappa}=\mathrm{u} 1 ; \operatorname{sigma}=\mathrm{u} 1 ; \mathrm{ss}=\mathrm{u} 1 ;$

do $\mathrm{k}=1$ to $\mathrm{n} 1$;

do $\mathrm{j}=1$ to $n$;

$\mathrm{x} 1[\mathrm{j}, \mathrm{k}]=\operatorname{ranuni}(134890+\mathrm{k}) ;$

$\mathrm{um}[\mathrm{j}, \mathrm{k}]=$ floor $(\mathrm{n} * \mathrm{x} 1[\mathrm{j}, \mathrm{k}]+1)$;

$\mathrm{x} 3[\mathrm{j}, \mathrm{k}]=\mathrm{x}[\mathrm{um}[\mathrm{j}, \mathrm{k}]] ;$ 
$\mathrm{c}[\mathrm{j}, \mathrm{k}]=\cos (\mathrm{x} 3[\mathrm{j}, \mathrm{k}]) ;$

$\mathrm{s}[\mathrm{j}, \mathrm{k}]=\sin (\mathrm{x} 3[\mathrm{j}, \mathrm{k}])$;

end;

$\mathrm{z} 1[\mathrm{k}]=\mathrm{c}[+, \mathrm{k}] / \mathrm{n} ; \mathrm{z} 2[\mathrm{k}]=\mathrm{s}[+, \mathrm{k}] / \mathrm{n} ;$

$\operatorname{rbg}[\mathrm{k}]=\operatorname{sqrt}\left(\mathrm{z} 1[\mathrm{k}]^{* *} 2+\mathrm{z} 2[\mathrm{k}]^{* *} 2\right) ;$

$\operatorname{ml} 1[\mathrm{k}]=2^{*} \operatorname{rbg}[\mathrm{k}]+\mathrm{rbg}[\mathrm{k}]^{* *} 3+5^{*}\left(\mathrm{rbg}[\mathrm{k}]^{* *} 5\right) / 6 ;$

$\mathrm{ml} 2[\mathrm{k}]=-0.4+1.39^{*} \mathrm{rbg}[\mathrm{k}]+0.43 /(1-\mathrm{rbg}[\mathrm{k}]) ;$

$\mathrm{ml} 3[\mathrm{k}]=1 /\left(\mathrm{rbg}[\mathrm{k}]^{* *} 3+4^{*} \mathrm{rbg}[\mathrm{k}]^{* *} 2+3^{*} \mathrm{rbg}[\mathrm{k}] ;\right.$

if $\operatorname{rbg}[\mathrm{k}]<0.53$ then $\mathrm{kml}[\mathrm{k}]=\operatorname{ml} 1[\mathrm{k}]$;

else

if $\operatorname{rbg}[\mathrm{k}]>=0.85$ then $\mathrm{kml}[\mathrm{k}]=\mathrm{ml} 3[\mathrm{k}]$;

else $k m l[k]=m l 2[k]$;

$\mathrm{kk} 1[\mathrm{k}]=\max \left(\left(\mathrm{kml}[\mathrm{k}]-2 /\left(\mathrm{n}^{*} \mathrm{kml}[\mathrm{k}]\right) 0,0\right)\right.$;

$\mathrm{kk} 2[\mathrm{k}]=\left((\mathrm{n}-1)^{* *} 3\right)^{*}\left(\mathrm{kml}[\mathrm{k}] /\left(\mathrm{n}^{* *} 3+\mathrm{n}\right)\right)$;

if $\mathrm{kml}[\mathrm{k}]<2$ then $\operatorname{kest}[\mathrm{k}]=\mathrm{kk} 1[\mathrm{k}]$;

else kest $[\mathrm{k}]=\mathrm{kk} 2[\mathrm{k}]$;

if $<=15$ then $\operatorname{kappa}[\mathrm{k}]=\operatorname{kest}[\mathrm{k}]$;

else kappa $[\mathrm{k}]=\mathrm{kml}[\mathrm{k}]$;

$\operatorname{sigma}[\mathrm{k}]=1 / \operatorname{sqrt}\left(\mathrm{n}^{*} \mathrm{rbg}[\mathrm{k}]^{*} \mathrm{kappa}[\mathrm{k}]\right)$;

end;

do $\mathrm{kk}=1$ to $\mathrm{n} 1$;

do $\mathrm{j}=1$ to $\mathrm{n}$;

$\mathrm{c} 2[\mathrm{j}, \mathrm{kk}]=(\mathrm{c}[\mathrm{j}, \mathrm{kk}]-\mathrm{c}[+, \mathrm{kk}]) \# \# 2$;

$\mathrm{s} 2[\mathrm{j}, \mathrm{kk}]=(\mathrm{s}[\mathrm{j}, \mathrm{kk}]-\mathrm{s}[+, \mathrm{kk}]) \# \# 2$;

$\mathrm{sc}[\mathrm{j}, \mathrm{kk}]=(\mathrm{c}[\mathrm{j}, \mathrm{kk}]-\mathrm{c}[+, \mathrm{kk}]) \#(\mathrm{~s}[\mathrm{j}, \mathrm{kk}]-\mathrm{s}[+, \mathrm{kk}]) ;$ 
end;

$\mathrm{u} 1[\mathrm{kk}]=\mathrm{c} 2[+, \mathrm{kk}] / \mathrm{n} ; \mathrm{u} 2[\mathrm{kk}]=\mathrm{s} 2[+, \mathrm{kk}] / \mathrm{n} ;$

$\mathrm{u} 12[\mathrm{kk}]=\mathrm{sc}[+, \mathrm{kk}] / \mathrm{n}$

$\mathrm{b}[\mathrm{kk}]=(\mathrm{u} 1[\mathrm{kk}]-\mathrm{u} 2[\mathrm{kk}]) /(2 * \mathrm{u} 12[\mathrm{kk}])-(((\mathrm{u} 1[\mathrm{kk}]-\mathrm{u} 2[\mathrm{kk}]) * * 2) /$

$\left.\left(4^{*}\left(\mathrm{u} 12[\mathrm{kk}]^{* *} 2\right)\right)+1\right)^{* *} 0.5$

$\mathrm{t} 1[\mathrm{kk}]=\left(1+\mathrm{b}[\mathrm{kk}]^{* *} 2\right)^{* *} 0.5 /\left(\left(\left(\mathrm{b}[\mathrm{kk}]^{* *} 2\right)^{*} \mathrm{u} 1[\mathrm{kk}]+2^{*} \mathrm{~b}[\mathrm{kk}]^{*} \mathrm{u} 12[\mathrm{kk}]+\mathrm{u} 2[\mathrm{kk}]\right)^{* *} 0.5\right)$;

$\mathrm{t} 2[\mathrm{kk}]=\left(1+\mathrm{b}[\mathrm{kk}]^{* *} 2\right)^{* *} 0.5 /\left(\mathrm{u} 1[\mathrm{kk}]-2^{*} \mathrm{~b}[\mathrm{kk}]^{*} \mathrm{u} 12[\mathrm{kk}]+\mathrm{u} 2[\mathrm{kk}]^{*}\left(\mathrm{~b}[\mathrm{kk}]^{* *} 2\right)\right)^{* *} 0.5$

$\mathrm{w} 1[\mathrm{kk}]=\left(\left(\mathrm{b}[\mathrm{kk}]^{* *} 2\right)^{*} \mathrm{t} 1[\mathrm{kk}]+\mathrm{t} 2[\mathrm{kk}]\right) /\left(1+\mathrm{b}[\mathrm{kk}]^{* *} 2\right)$;

$\mathrm{w} 2[\mathrm{kk}]=\left(\mathrm{t} 1[\mathrm{kk}]+\mathrm{t} 2[\mathrm{kk}]^{*}\left(\mathrm{~b}[\mathrm{kk}]^{* *} 2\right)\right) /\left(1+\mathrm{b}[\mathrm{kk}]^{* *} 2\right) ;$

$\mathrm{w} 12[\mathrm{kk}]=\mathrm{b}[\mathrm{kk}]^{*}(\mathrm{t} 1[\mathrm{kk}]-\mathrm{t} 2[\mathrm{kk}]) /\left(1+\mathrm{b}[\mathrm{kk}]^{* *} 2\right)$; z02);

$\mathrm{cb}[\mathrm{kk}]=\mathrm{z} 01-\left(\mathrm{v} 01{ }^{*} \mathrm{w} 1[\mathrm{kk}]+\mathrm{v} 012 * \mathrm{w} 12[\mathrm{kk}]\right) *(\mathrm{z} 1[\mathrm{kk}]-\mathrm{z} 01)-\left(\mathrm{v} 01{ }^{*} \mathrm{w} 12[\mathrm{kk}]+\mathrm{v} 012^{*} \mathrm{w} 2[\mathrm{kk}]\right) *(\mathrm{z} 2[\mathrm{kk}]-$ z02);

$\mathrm{sb}[\mathrm{kk}]=\mathrm{z} 02-(\mathrm{v} 012 * \mathrm{w} 1[\mathrm{kk}]+\mathrm{v} 02 * \mathrm{w} 12[\mathrm{kk}]) *(\mathrm{z} 1[\mathrm{kk}]-\mathrm{z} 01)-(\mathrm{v} 012 * \mathrm{w} 12[\mathrm{kk}]+\mathrm{v} 02 * \mathrm{w} 2[\mathrm{kk}]) *(\mathrm{z} 2[\mathrm{kk}]-$

$\operatorname{cbg}[\mathrm{kk}]=\mathrm{cb}[\mathrm{kk}]^{*}\left(\left(\mathrm{cb}[\mathrm{kk}]^{* *} 2+\mathrm{sb}[\mathrm{kk}]^{* *} 2\right)^{* *}(-0.5)\right) ;$

$\operatorname{sbg}[\mathrm{kk}]=\operatorname{sb}[\mathrm{kk}]^{*}\left(\left(\mathrm{cb}[\mathrm{kk}]^{* *} 2+\mathrm{sb}[\mathrm{kk}]^{* *} 2\right)^{* *}(-0.5)\right)$;

$\mathrm{q}[\mathrm{kk}]=\operatorname{atan}(\operatorname{sbg}[\mathrm{kk}] / \operatorname{cbg}[\mathrm{kk}])$;

$\mathrm{q} 1[\mathrm{kk}]=\mathrm{q}[\mathrm{kk}]+\mathrm{pi} ;$

$\mathrm{q} 2[\mathrm{kk}]=\mathrm{q}[\mathrm{kk}]+2^{*} \mathrm{pi}$

$/$ os elementos do vetor muboos são as estimativas de $\mu$ para cada amostra bootstrap*/

if $\operatorname{sbg}[\mathrm{kk}]>0 \& \operatorname{cbg}[\mathrm{kk}]>0$ then muboos $[\mathrm{kk}]=\mathrm{q}[\mathrm{kk}]$;

else

if $\operatorname{cbg}[\mathrm{kk}]>0 \& \operatorname{sbg}[\mathrm{kk}]<0$ then muboos $[\mathrm{kk}]=\mathrm{q} 2[\mathrm{kk}]$;

else muboos[kk]=q1[kk];

finish; 


\section{Progrma INTBOOTS.SAS}

Este programa calcula intervalo de confiança para $\mu$ pelo método bootstrap.

proc iml;

$\mathrm{pi}=3.14159$;

$\mathrm{y}=\{294,301,329,315,277,281,254,245,272,242,177$,

$257,177,229,250,166,232,245,224,186\}$;

$\mathrm{r}=\{\mathrm{y}\} ;$

create ydados from $\mathrm{y}[$ colmane $=\mathrm{r}]$;

append from y;

$\mathrm{x}=\mathrm{y}$;

$\mathrm{n} 1=200$;

run boost;

gamab=muboos-mu $;$ arq $0=$ gamab;

$\mathrm{r} 0=\{$ gamab $\} ;$

create dados 1 from arq $0[\operatorname{colname}=\mathrm{r} 0]$;

append from arq0;

proc sort data $=$ dados 1 out data $=$ dadoss;

by gamab ; proc iml;

use dadoss;

read all varmuboos;

alpha $=0.05$;

$\mathrm{l}=$ floor $(\mathrm{n} 1 *$ alpha*0.5+0.5);

$\mathrm{m}=\mathrm{n} 1-\mathrm{l}$

le $=$ gamab[1+1];

re=gamab $[\mathrm{m}]$; use ydados; read all vary; run media;

$\mathrm{pi}=3.141516$; 
lif $=\mathrm{le}^{*} 180 / \mathrm{pi}+$ media;

lsf $=\mathrm{re}^{*} 180 /$ pi + media;

print "Os limites em graus";

print media lif lsf ;

run;

quit;

Este programa testa $H_{0}: \mu=\mu_{0}$ pelo método bootstrap.

libname novo 'c:';

proc iml;

$\mathrm{pi}=3.14159$;

$\mathrm{mu} 00=3.6$;

$\mathrm{y}=\{294,301,329,315,277,281,254,245,272,242,177$,

$257,177,229,250,166,232,245,224,186\}$;

run media;

media $=$ te;

est $=\operatorname{abs}(\sin ($ media-mu00)/sigma $)$;

print est;

$\mathrm{x}=\mathrm{y}^{*} \mathrm{pi} / 180-($ media-mu00);

$\mathrm{n} 1=200$

run boost;

mboos $=$ muboos;

$\mathrm{r}=\mathrm{rbg}$;

do $\mathrm{k}=1$ to $\mathrm{n} 1$;

$\operatorname{delta}[\mathrm{k}]=(1-\mathrm{r} 2[\mathrm{k}]) /\left(2 *^{*}[\mathrm{k}]\right)$;

$\operatorname{sigma}[\mathrm{k}]=\operatorname{sqrt}(\operatorname{delta}[\mathrm{k}] / \mathrm{n})$; 


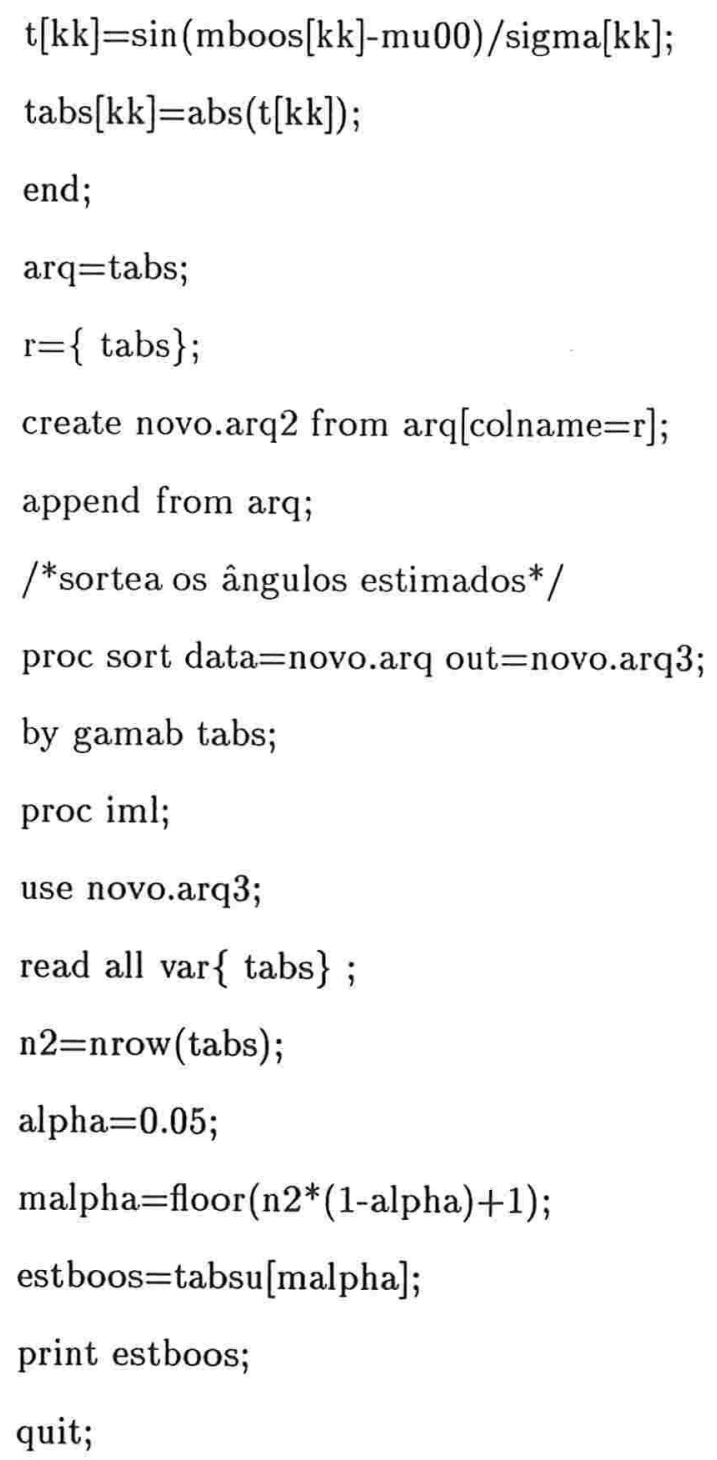

\section{Programa ESTVON.SAS}

Este módulo calcula as estimativas de $\mu$ e $\kappa$ em uma amostra da distribuição de von Mises.

start estvon;

$\mathrm{pi}=3.14159$;

$\mathrm{x}=\mathrm{y}^{*} \mathrm{pi} / 180$;

$\mathrm{n}=\operatorname{nrow}(\mathrm{x})$; 
$\mathrm{c} 0=\mathrm{j}(\mathrm{n}, 1) ; \mathrm{s} 0=\mathrm{c} 0 ; \mathrm{x} 01=\mathrm{c} 0 ; \mathrm{x} 02=\mathrm{c} 0 ; \mathrm{x} 012=\mathrm{c} 0$;

do $\mathrm{j}=1$ to $\mathrm{n}$;

$\mathrm{c} 0[\mathrm{j}]=\cos (\mathrm{x}[\mathrm{j}]) ; \mathrm{s} 0[\mathrm{j}]=\sin (\mathrm{x}[\mathrm{j}]) ;$

end;

$\mathrm{z} 01=\mathrm{c} 0[+] / \mathrm{n} ; \mathrm{z} 02=\mathrm{s} 0[+] / \mathrm{n} ;$

$\mathrm{q} 0=\operatorname{atan}(\mathrm{z} 02 / \mathrm{z} 01)$;

$\mathrm{q} 01=\mathrm{q} 0+\mathrm{pi} ;$

$\mathrm{q} 02=\mathrm{q} 0+2^{*} \mathrm{pi} ;$

if $\mathrm{z} 02>0 \& \mathrm{z} 01>0$ then $\mathrm{mu} 0=\mathrm{q} 0$;

else

if $\mathrm{z} 02<0 \& \mathrm{z} 01>0$ then $\mathrm{mu} 0=\mathrm{q} 02$;

else $\mathrm{mu} 0=\mathrm{q} 01$;

$\operatorname{rbar}=\operatorname{sqrt}\left(\mathrm{z}_{01} 1^{* *} 2+\mathrm{z} 02^{* *} 2\right) ;$

$\operatorname{ml} 1=2 * \operatorname{rbar}+\operatorname{rbar}^{* *} 3+5^{*}\left(\operatorname{rbar}^{* *} 5\right) / 6 ;$

$\mathrm{ml} 2=-0.4+1.39 *$ rbar $+0.43 /(1-$ rbar $)$;

$\mathrm{ml} 3=1 /\left(\operatorname{rbar}^{* *} 3+4^{*} \mathrm{rbar}^{* *} 2+3 * \mathrm{rbar}\right) ;$

if $\mathrm{rbar}<0.53$ then $\mathrm{kml}=\mathrm{ml}$;

else if $\mathrm{rbar}>=0.85$ then $\mathrm{kml}=\mathrm{ml} 3$;

else $\mathrm{kml}=\mathrm{ml} 2$

$\mathrm{kk} 1=\max ((\mathrm{kml}-2 /(\mathrm{n} * \mathrm{kml})), 0)$;

$\mathrm{kk} 2=\left((\mathrm{n}-1)^{* *} 3\right)^{*}(\mathrm{kml} /(\mathrm{n} * * 3+\mathrm{n})) ;$

if $\mathrm{kml}<2$ then kest=kk1;

else kest $=\mathrm{kk} 2$;

if $\mathrm{n}<=15$ then kkappa= kest;

else kkappa $=\mathrm{kml}$;

vsigma $=1 / \operatorname{sqrt}\left(\left(\mathrm{n}^{*}\right.\right.$ rbar*kappa $\left.)\right)$; 
vdelta $=1 / \operatorname{sqrt}(($ rbar*kappa $))$;

print ' os estimadores são: ' mu0, kkappa;

finish;

Programa BOOTSP.SAS

Gera amostras bootstrap da distribuição von Mises.

libname novo 'c:';

proc iml;

start boostp;

$\mathrm{pi}=3.141516$;

$\mathrm{x}=\mathrm{y}^{*} \mathrm{pi} / 180$;

$\mathrm{n}=\operatorname{nrow}(\mathrm{x})$;

$\mathrm{cb}=\mathrm{j}(\mathrm{n} 1,1) ; \mathrm{sb}=\mathrm{j}(\mathrm{n} 1,1) ; \mathrm{q}=\mathrm{j}(\mathrm{n} 1,1) ;$

$q 1=j(n 1,1) ; q 2=j(n 1,1) ;$ muboos $=j(n 1,1) ;$

$\operatorname{cbg}=\mathrm{j}(\mathrm{n} 1,1) ; \operatorname{sbg}=\mathrm{j}(\mathrm{n} 1,1) ;$

$x 1=j(n, n 1) ; x 2=j(n, n 1) ; x 3=j(n, n 1) ;$

$\mathrm{um}=\mathrm{j}(\mathrm{n}, \mathrm{n} 1) ; \mathrm{c}=\mathrm{j}(\mathrm{n}, \mathrm{n} 1) ; \mathrm{s}=\mathrm{j}(\mathrm{n}, \mathrm{n} 1)$;

$\mathrm{z} 1=\mathrm{j}(\mathrm{n} 1,1) ; \mathrm{z} 2=\mathrm{j}(\mathrm{n} 1,1) ;$

$c 2=j(n, n 1) ; s c=j(n, n 1)$;

$\mathrm{s} 2=\mathrm{j}(\mathrm{n}, \mathrm{n} 1) ; \mathrm{u} 1=\mathrm{j}(\mathrm{n} 1,1) ; \mathrm{u} 2=\mathrm{j}(\mathrm{n} 1,1) ; \mathrm{u} 12=\mathrm{j}(\mathrm{n} 1,1)$;

$\mathrm{b}=\mathrm{u} 1 ; \mathrm{t} 1=\mathrm{u} 1 ; \mathrm{t} 2=\mathrm{u} 1 ; \mathrm{w} 1=\mathrm{u} 1 ; \mathrm{w} 2=\mathrm{u} 1 ; \mathrm{w} 12=\mathrm{u} 1 ;$

$\mathrm{rrb}=\mathrm{j}(\mathrm{n} 1,1) ; \mathrm{kkl} 1=\mathrm{rrb} ; \mathrm{kkl} 2=\mathrm{kkl} 1 ; \mathrm{kkl} 3=\mathrm{kkl} 2 ; \mathrm{kkl}=\mathrm{kkl} 3 ;$

kkes $=\mathrm{j}(\mathrm{n} 1,1) ; \mathrm{kkes} 1=\mathrm{kkes} ; \mathrm{kkes} 2=\mathrm{kkes} 1 ; \mathrm{kapa}=\mathrm{kkes} 2$;

$\mathrm{c} 0=\mathrm{j}(\mathrm{n}, 1) ; \mathrm{s} 0=\mathrm{c} 0 ; \mathrm{x} 01=\mathrm{c} 0 ; \mathrm{x} 02=\mathrm{c} 0 ; \mathrm{x} 012=\mathrm{c} 0 ;$

do $\mathrm{j}=1$ to $n$;

$\mathrm{c} 0[\mathrm{j}]=\cos (\mathrm{x}[\mathrm{j}]) ; \mathrm{s} 0[\mathrm{j}]=\sin (\mathrm{x}[\mathrm{j}]) ;$ 
end;

$\mathrm{z} 01=\mathrm{c} 0[+] / \mathrm{n} ; \mathrm{z} 02=\mathrm{s} 0[+] / \mathrm{n} ;$

$\mathrm{q} 0=\operatorname{atan}(\mathrm{z} 02 / \mathrm{z} 01)$;

$\mathrm{q} 01=\mathrm{q} 0+\mathrm{pi} ;$

$\mathrm{q} 02=\mathrm{q} 0+2^{*} \mathrm{pi}$

if $\mathrm{z} 02>0$ \& $\mathrm{z} 01>0$ then $\mathrm{mu} 0=\mathrm{q} 0$;

else

if $\mathrm{z} 02<0$ \& $\mathrm{z} 01>0$ then $\mathrm{mu} 0=\mathrm{q} 02$;

else $\mathrm{mu} 0=\mathrm{q} 01$;

$\operatorname{rbar}=\operatorname{sqrt}\left(\mathrm{z} 01^{* *} 2+\mathrm{z} 02^{* *} 2\right)$;

$\mathrm{klm} 1=2 *$ rbar $+\mathrm{rbar}^{* *} 3+5 * \mathrm{rbar}^{* *} 5 / 6$;

$\mathrm{k} \operatorname{lm} 2=-0.4+1.39 * \mathrm{rbar}+0.43 /(1-\mathrm{rbar})$;

$\mathrm{kl} 3=1 /\left(\operatorname{rbar}^{* *} 3-4 * \mathrm{rbar}^{* *} 2+3 *\right.$ rbar $)$

if $\operatorname{rbar}<0.53$ then $\mathrm{kl}=\mathrm{klm} 1$;

else if $\mathrm{rbar}>=0.53 \& \mathrm{rbar}<0.83$ then $\mathrm{kl}=\mathrm{kml}$;

else $\mathrm{kl}=\mathrm{kl} 3$;

$\operatorname{kes} 1=\max \left(\mathrm{kl}-2^{*}(\mathrm{n} * \mathrm{kl}) * *(-1), 0\right)$;

$\operatorname{kes} 2=\mathrm{kl}^{*}(\mathrm{n}-1)^{* *} 3 /\left(\mathrm{n}^{* *} 3+\mathrm{n}\right)$;

if $\mathrm{kl}<2$ then $\mathrm{kes}=\mathrm{kes} 1$;

else kes=kes2;

if $\mathrm{n}<=15$ then kkapa=kes;

else kkapa=kl; print kkapa mu0;

do $\mathrm{k}=1$ to $\mathrm{n}$;

$\mathrm{x} 01[\mathrm{k}]=(\mathrm{c} 0[\mathrm{k}]-\mathrm{z} 01) \# \# 2$;

$\mathrm{x} 02[\mathrm{k}]=(\mathrm{s} 0[\mathrm{k}]-\mathrm{z} 02) \# \# 2 ; \mathrm{x} 012[\mathrm{k}]=(\mathrm{c} 0[\mathrm{k}]-\mathrm{z} 01) \#(\mathrm{~s} 0[\mathrm{k}]-\mathrm{z} 02)$;

end; 


$$
\begin{aligned}
& \mathrm{u} 01=\mathrm{x} 01[+] / \mathrm{n} ; \mathrm{u} 02=\mathrm{x} 02[+] / \mathrm{n} ; \mathrm{u} 012=\mathrm{x} 012[+\mathrm{]} / \mathrm{n} ; \\
& \mathrm{b} 0=(\mathrm{u} 01-\mathrm{u} 02) /\left(2^{*} \mathrm{u} 012\right)-\left((\mathrm{u} 01-\mathrm{u} 02)^{* *} 2 /\left(4^{*}\left(\mathrm{u} 012^{* *} 2\right)\right)+1\right)^{* *} 0.5 ; \\
& \mathrm{t} 01=\left(\mathrm{u} 01 *(\mathrm{~b} 0 * * 2)+2^{*} \mathrm{~b} 00^{*} \mathrm{u} 012+\mathrm{u} 02\right)^{* *} 0.5 /\left(1+\mathrm{b} 0^{* *} 2\right)^{* *} 0.5 ; \\
& \mathrm{t} 02=\left(\mathrm{u} 01-2^{*} \mathrm{~b} 0 * \mathrm{u} 012+\mathrm{u} 02^{*}(\mathrm{~b} 0 * * 2)\right) /\left(1+\mathrm{b} 0^{* *} 2\right)^{* *} 0.5 ; \\
& \mathrm{v} 01=\left(\mathrm{t} 01^{*}\left(\mathrm{~b} 0^{* *} 2\right)+\mathrm{t} 02\right) /\left(1+\mathrm{b} 0^{* *} 2\right) ; \\
& \mathrm{v} 02=\left(\mathrm{t} 01+\mathrm{t} 02^{*}(\mathrm{~b} 0 * * 2)\right) /\left(1+\mathrm{b} 0^{* *} 2\right) ; \\
& \mathrm{v} 012=\mathrm{b} 0 *(\mathrm{t} 01-\mathrm{t} 02) /\left(1+\mathrm{b} 0^{* *} 2\right) ; \\
& \mathrm{kappa}=\mathrm{kkapa} ; \mathrm{pi}=3.14159 ; \mathrm{mu}=\mathrm{mu} 0 ;
\end{aligned}
$$$$
\mathrm{a}=1+\left(1+4^{*}\left(\mathrm{kappa}^{* *} 2\right)\right)^{* *} 0.5 ; \mathrm{bb}=\left(\mathrm{a}-\left(2^{*} \mathrm{a}\right){ }^{* *} 0.5\right) /\left(2^{*} \text { kappa }\right) \text {; }
$$$$
\mathrm{r}=\left(1+\mathrm{bb}^{* *} 2\right) /\left(2^{*} \mathrm{bb}\right) \text {; }
$$$$
\mathrm{uu} 1=\mathrm{j}(\mathrm{n}, 1) ; \mathrm{uu} 2=\mathrm{j}(\mathrm{n}, 1) ; \mathrm{uu} 3=\mathrm{j}(\mathrm{n}, 1) \text {; }
$$$$
\mathrm{ze}=\mathrm{uu} 1 ; \mathrm{f}=\mathrm{uu} 1 ; \mathrm{cc}=\mathrm{uu} 1 ; \operatorname{teta}=\mathrm{uu} 1 ; \operatorname{teta} 1=\mathrm{uu} 1 ;
$$

/*Gera amostras bootstrp pamétrico */

do $\mathrm{k}=1$ to $\mathrm{n} 1$;

do $\mathrm{j}=1$ to $n$;

p1:

uu1[j] =ranuni $(2344+\mathrm{k})$;

$\mathrm{uu} 2[\mathrm{j}]=\operatorname{ranuni}(1344+\mathrm{k})$;

uu3[j] $=\operatorname{ranuni}(26781234+\mathrm{k})$;

$\mathrm{ze}[\mathrm{j}]=\cos \left(\mathrm{pi}^{*} \mathrm{uu} 1[\mathrm{j}]\right)$;

$\mathrm{f}[\mathrm{j}]=\left(1+\mathrm{r}^{*} \mathrm{ze}[\mathrm{j}]\right) /(\mathrm{r}+\mathrm{ze}[\mathrm{j}])$;

$\mathrm{c}[\mathrm{j}]=\operatorname{kappa}^{*}(\mathrm{r}-\mathrm{f}[\mathrm{j}])$;

if $c c[j]^{*}(2-c c[j])>u u 2[j]$ then goto $p 2$;

else if $\log (\mathrm{cc}[\mathrm{j}] / \mathrm{uu} 2[\mathrm{j}])<(\mathrm{cc}[\mathrm{j}]-1)$ then goto $\mathrm{p} 1$;

else goto $\mathrm{p} 2$;

$\mathrm{p} 2: \operatorname{teta}[\mathrm{j}]=\bmod \left(\left(\operatorname{sign}(\mathrm{uu} 3[\mathrm{j}]-0.5)^{*} \operatorname{arcos}(\mathrm{f}[\mathrm{j}])+\mathrm{mu}\right), 2^{*} \mathrm{pi}\right)$; 
if teta[j] $<0$ then $\operatorname{teta} 1[\mathrm{j}]=\operatorname{teta}[\mathrm{j}]+2 * \mathrm{pi}$;

else teta $1[\mathrm{j}]=$ teta $[\mathrm{j}]$;

end;

$\mathrm{x} 3[, \mathrm{k}]=$ teta $1 ;$

end;

$\mathrm{c}=\cos (\mathrm{x} 3)$;

$\mathrm{s}=\sin (\mathrm{x} 3) ;$

do $\mathrm{k}=1$ to $\mathrm{n} 1$;

$\mathrm{z} 1[\mathrm{k}]=\mathrm{c}[+, \mathrm{k}] / \mathrm{n} ; \mathrm{z} 2[\mathrm{k}]=\mathrm{s}[+, \mathrm{k}] / \mathrm{n} ;$

end;

do $\mathrm{kk}=1$ to $\mathrm{n} 1$;

do $\mathrm{j}=1$ to $n$;

$\mathrm{c} 2[\mathrm{j}, \mathrm{kk}]=(\mathrm{c}[\mathrm{j}, \mathrm{kk}]-\mathrm{c}[+, \mathrm{kk}]) \# \# 2$;

$\mathrm{s} 2[\mathrm{j}, \mathrm{kk}]=(\mathrm{s}[\mathrm{j}, \mathrm{kk}]-\mathrm{s}[+, \mathrm{kk}]) \# \# 2$;

$\mathrm{sc}[\mathrm{j}, \mathrm{kk}]=(\mathrm{c}[\mathrm{j}, \mathrm{kk}]-\mathrm{c}[+, \mathrm{kk}]) \#(\mathrm{~s}[\mathrm{j}, \mathrm{kk}]-\mathrm{s}[+, \mathrm{kk}])$;

end;

$\mathrm{u} 1[\mathrm{kk}]=\mathrm{c} 2[+, \mathrm{kk}] / \mathrm{n}$;

$\mathrm{u} 2[\mathrm{kk}]=\mathrm{s} 2[+, \mathrm{kk}] / \mathrm{n}$;

$\mathrm{u} 12[\mathrm{kk}]=\mathrm{sc}[+, \mathrm{kk}] / \mathrm{n}$;

$\mathrm{b}[\mathrm{kk}]=(\mathrm{u} 1[\mathrm{kk}]-\mathrm{u} 2[\mathrm{kk}]) /(2 * \mathrm{u} 12[\mathrm{kk}])-(((\mathrm{u} 1[\mathrm{kk}]-\mathrm{u} 2[\mathrm{kk}]) \# \# 2) /$

$\left.\left(4^{*}(\mathrm{u} 12[\mathrm{kk}] \# \# 2)\right)+1\right) \# \# 0.5$

$\mathrm{t} 1[\mathrm{kk}]=(1+\mathrm{b}[\mathrm{kk}] \# \# 2)^{* *} 0.5 /\left(\left((\mathrm{b}[\mathrm{kk}] \# \# 2)^{*} \mathrm{u} 1[\mathrm{kk}]\right.\right.$

$\left.\left.+2 * \mathrm{~b}[\mathrm{kk}]^{*} \mathrm{u} 12[\mathrm{kk}]+\mathrm{u} 2[\mathrm{kk}]\right) \# \# 0.5\right)$;

$\mathrm{t} 2[\mathrm{kk}]=(1+\mathrm{b}[\mathrm{kk}] \# \# 2)^{* *} 0.5 /\left(\mathrm{u} 1[\mathrm{kk}]-2^{*} \mathrm{~b}[\mathrm{kk}]{ }^{*} \mathrm{u} 12[\mathrm{kk}]\right.$

$\left.+\mathrm{u} 2[\mathrm{kk}]^{*}(\mathrm{~b}[\mathrm{kk}] \# \# 2)\right) \# \# 0.5$;

$\mathrm{w} 1[\mathrm{kk}]=((\mathrm{b}[\mathrm{kk}] \# \# 2) * \mathrm{t} 1[\mathrm{kk}]+\mathrm{t} 2[\mathrm{kk}]) /(1+\mathrm{b}[\mathrm{kk}] \# \# 2)$; 


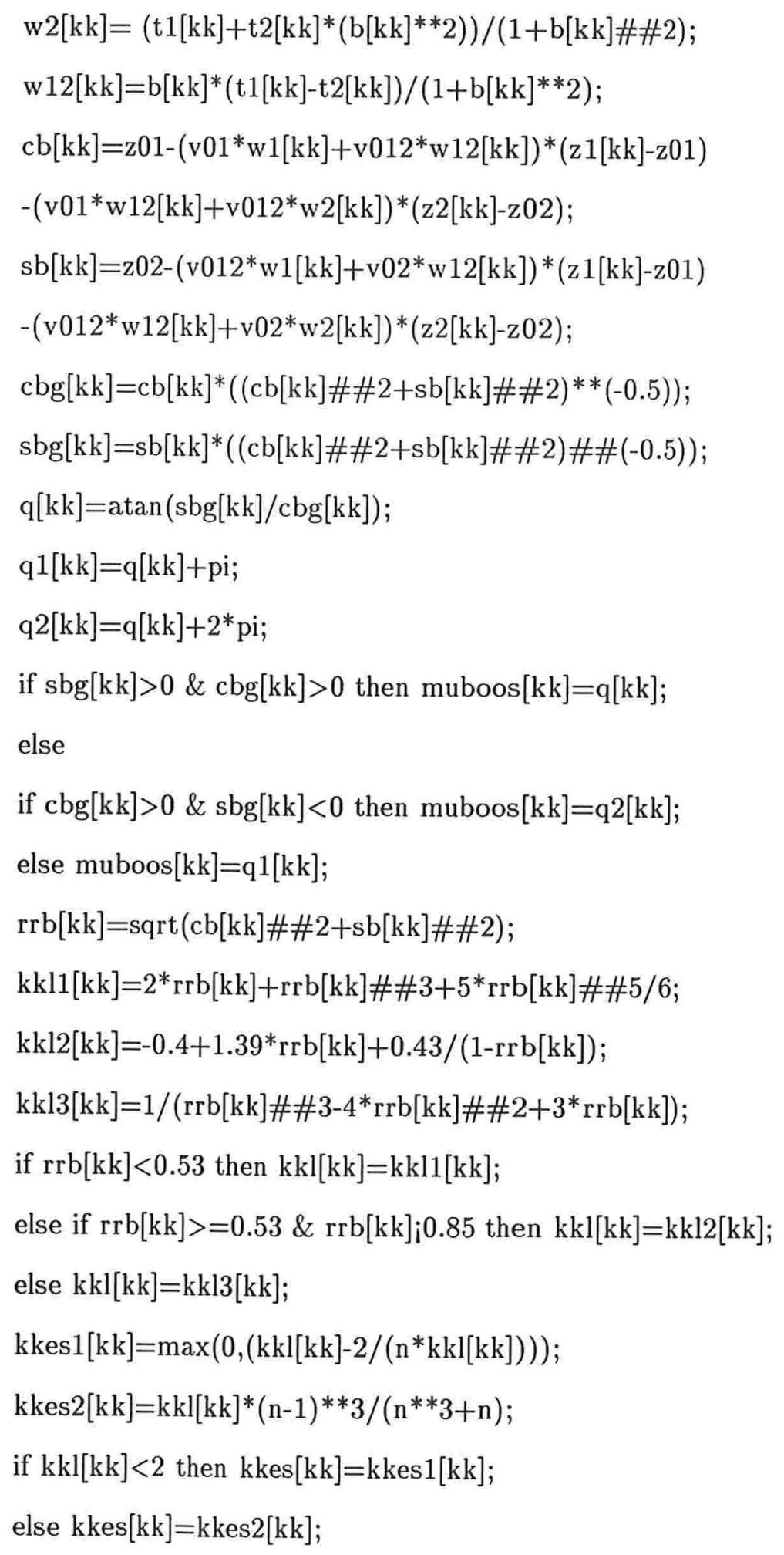


if $\mathrm{n}<=15$ then kapa[kk]=kkes[kk];

else kapa[kk]=kkl[kk];

end;

arq $=$ muboos || $\mathrm{kapa}$;

$\mathrm{r}=\{$ muboos kkapa $\}$;

create novo.arq from arq[ $\operatorname{colname}=\mathrm{r}]$;

append from arq;

finish;

Programa PRINC1.SAS

Este programa calcula a estatística para testar $H_{0}: \mu=\mu_{0}$ em uma amostra da distribuição de von Mises pelo método bootstrap (para $\mathrm{n}$ pequeno)

proc iml;

$\mathrm{n}=10 ; \quad / *$ tamanho da amostra */

kappa $=1 ; \quad / *$ parâmetro de concentração */

amedio $=45 ; \quad \quad / *$ ângulo médio em graus */

run von;

$\mathrm{y}=$ teta $1 ; /$ vetor de dados da distribuição $\operatorname{VM}(45,1) * /$

run estvon;

med $=\mathrm{mu} 0$;

ssigma $=$ sigma

est $=\sin ($ med-amedio*3.14159/180)/ssigma;

abest=abs(est);

print abest;

$\mathrm{x}=\mathrm{y}-($ med-amedio*3.14159/180);

n1 $=200 ; \quad / *$ número de amostras bootstrap */

run boostp; 
mmuboos=muboos;

ssigmm $=$ sigma;

do $\mathrm{i}=1$ to $\mathrm{n} 1$;

$\mathrm{ss}[\mathrm{i}]=\operatorname{abs}(\sin (\mathrm{mmuboos}[\mathrm{i}]-\mathrm{med}) / \operatorname{ssigmm}[\mathrm{i}])$;

end;

$\operatorname{arq}=\mathrm{ss}$;

$\mathrm{r}=\{$ tabsu $\}$

create novo.arq from arq[ $\operatorname{colname}=\mathrm{r}]$;

append from arq;

proc sort data $=$ novo. arq out $=$ novo.. arq 1 ;

by tabsu;

proc iml;

use novo.arq1;

read all $\operatorname{var}\{$ tabsu $\}$

$\mathrm{n} 2=\operatorname{nrow}(\mathrm{tabsu})$

alpha $=0.05$;

malpha $=$ floor $(\mathrm{n} 2 *(1$-alpha $)+1)$;

umalpha $=$ tabsu[malpha];

print umalpha;

quit; 


\section{INTBOOP.SAS}

Este programa calcula o intervalo de confiança bootstrap para o parâmentro de concentração $\kappa$.

proc iml;

libname novol 'c:';

$\mathrm{y}=\{28.60,191.83,271.57,43.11,279.39,51.79,80.90,69.39,18.47,118.92\} ;$

$\mathrm{n} 1=200$;

run boostp;

proc sort data $=$ novo1. arq out $=$ novo1. arq $1 ;$

by kkapa;

proc iml;

use novol.arq1;

read all var $\{$ muboos kkapa $\}$;

n1=nrow $($ kkapa $)$;

alpha $=0.05$

$\mathrm{l}=$ floor $(\mathrm{n} 1 *$ alpha*0.5+0.5);

$\mathrm{m}=\mathrm{n} 1-\mathrm{l}$

lif=kkapa $[1+1]$;

lis $=\mathrm{kkapa}[\mathrm{m}]$;

print "Os limites em graus";

print lif lis ;

quit;

\section{Progrma TESPM.SAS}

Este programa calcula as estatísticas para comparar as direções médias de duas amostras. proc iml;

$\mathrm{y}=\{5,15,15,20,20,35,40,45,45,50,65,70,115,115,150,220,225$, 
$235,245,250,255,270,280,280,295,295,300,305$,

$310,310,315,320,320,320,325,330,330,340,340,355\} ; \quad \quad{ }^{*}$ dados da amostra $1^{*} /$

run media;

$\mathrm{n} 1=\mathrm{n}$;

te $1=$ te;

delta $1=$ delta;

sigma1=sigma;

$\mathrm{y}=\{5,10,10,15,35,45,50,50,55,60,60,60,65,70,110,185$,

$215,305,320,325,330,345,350,350,355\} ; \quad / *$ dados da amostra $2 * /$

run media;

$\mathrm{n} 2=\mathrm{n} ;$

te $2=$ te;

delta2=delta;

sigma2=sigma;

$\mathrm{cp}=\mathrm{n} 1 * \cos (\mathrm{te} 1)+\mathrm{n} 2 * \cos (\mathrm{te} 2) ;$

$\mathrm{sp}=\mathrm{n} 1 * \sin (\mathrm{te} 1)+\mathrm{n} 2 * \sin (\mathrm{te} 2) ;$

$\mathrm{rp}=\operatorname{sqrt}\left(\mathrm{cp}^{* *} 2+\mathrm{sp}^{* *} 2\right)$;

$\operatorname{delta} 0=(\mathrm{n} 1 * \operatorname{delta} 1+\mathrm{n} 2 * \operatorname{delta} 2) /(\mathrm{n} 1+\mathrm{n} 2) ;$

MetodoP $=2 *(\mathrm{n} 1+\mathrm{n} 2+-\mathrm{rp})$;

$\mathrm{cm}=\cos (\mathrm{te} 1) /(\operatorname{sigma} 1 * * 2)+\cos (\mathrm{te} 2) /(\operatorname{sigma} 2 * * 2) ;$

$\operatorname{sm}=\sin ($ te 1$) /(\operatorname{sigma} 1 * * 2)+\sin ($ te 2$) /(\operatorname{sigma} 2 * * 2) ;$

$\mathrm{rm}=\operatorname{sqrt}\left(\mathrm{cm}^{* *} 2+\mathrm{sm}^{* * 2}\right)$;

MetodoM $=2^{*}(1 /(\operatorname{sigma} 1 * * 2)+1 /(\operatorname{sigma} 2 * * 2) ;$

delmax $=\max ($ delta1,delta2);

delmin $=\min ($ delta $1, \operatorname{delta} 2) ;$

qdelta $=$ delmax $/$ delmin; 
if qdelta $<=4$ then print MetodoP;

else print metodom;

p1value $=1-$ probchi(metodop,2);

p2value =1-probchi(metodom,2);

if qdelta $<=4$ then print plvalue;

else print p2value;

quit;

\section{Programa TPCONVM.SAS}

Este módulo calcula a estatística para teste de igualdade dos parâmetros de concentração de três distribuições von Mises.

proc iml;

start testk;

$\mathrm{pi}=3.14159$;

$\mathrm{d} 1=\operatorname{abs}(\sin (\mathrm{y} 1-251.064 * \mathrm{pi} / 180))$;

$\mathrm{d} 2=\operatorname{abs}\left(\sin \left(\mathrm{y} 2-129.718^{*} \mathrm{pi} / 180\right)\right)$;

$\mathrm{d} 3=\operatorname{abs}\left(\sin \left(\mathrm{y} 2-57.694^{*} \mathrm{pi} / 180\right)\right) ;$

$\mathrm{n} 1=$ nrow $(\mathrm{d} 1)$;

$\mathrm{n} 2=\operatorname{nrow}(\mathrm{d} 2) ;$

$\mathrm{n} 3=$ nrow $(\mathrm{d} 3)$;

$\mathrm{n}=\mathrm{n} 1+\mathrm{n} 2+\mathrm{n} 3 ;$

$\mathrm{s} 1=\mathrm{d} 1[+] / \mathrm{n} 1 ;$

$\mathrm{s} 2=\mathrm{d} 2[+] / \mathrm{n} 2 ;$

$\mathrm{s} 3=\mathrm{d} 3[+] / \mathrm{n} 3 ;$

$\mathrm{d}=\left(\mathrm{n} 1 * \mathrm{~s} 1+\mathrm{n} 2{ }^{*} \mathrm{~s} 2+\mathrm{n} 3 * \mathrm{~s} 3\right) / \mathrm{n} ;$

$\mathrm{dn}=\mathrm{n} 1 *(\mathrm{~s} 1-\mathrm{d}) * * 2+\mathrm{n} 22^{*}(\mathrm{~s} 2-\mathrm{d}) * * 2+\mathrm{n} 3^{*}(\mathrm{~s} 3-\mathrm{d}) * * 2 ; \mathrm{dd} 1=\mathrm{sum}((\mathrm{d} 1-\mathrm{s} 1) \# \# 2) ;$

$\mathrm{dd} 2=\operatorname{sum}((\mathrm{d} 2-\mathrm{s} 2) \# \# 2) ;$ 
$\mathrm{dd} 3=\operatorname{sum}((\mathrm{d} 3-\mathrm{s} 3) \# \# 2)$

$\mathrm{nu}=\mathrm{dd} 1+\mathrm{dd} 2+\mathrm{dd} 3 ;$

$\mathrm{fr}=(\mathrm{n}-3) * \operatorname{dn} /(2 * \mathrm{nu})$

Pvalue=1-probf $(\mathrm{fr}, 2,(\mathrm{n}-3))$;

print fr Pvalue;

finish;

quit;

\section{Programa TEM2VM.SAS}

Programa que calcula a estatística para testar $H_{0}: \mu_{1}=\mu_{2}=\mu_{3}$ na distribuição von Mises. proc iml;

$\mathrm{y}=\mathrm{y} 1 ; \quad / *$ dados da amostra $1 * /$

run estvon;

$\mathrm{mu} 1=\mathrm{mu} 0 ;$

$\mathrm{r} 1=\mathrm{rbar} ;$

$\mathrm{n} 1=\mathrm{n}$;

kappa1=kappa;

$\mathrm{y}=\mathrm{y} 2 ; \quad \quad \quad{ }^{*}$ dados da amostra $2^{*} /$

run estvon;

$\mathrm{mu} 2=\mathrm{mu} 0 ;$

$\mathrm{r} 2=$ rbar;

$\mathrm{n} 2=\mathrm{n} ;$

kappa2=kappa;

$\mathrm{y}=\mathrm{y} 3 ; \quad /^{*}$ dados da amostra $3^{*} /$

run estvon;

$\mathrm{mu} 3=\mathrm{mu} 0 ;$

r3=rbar; 


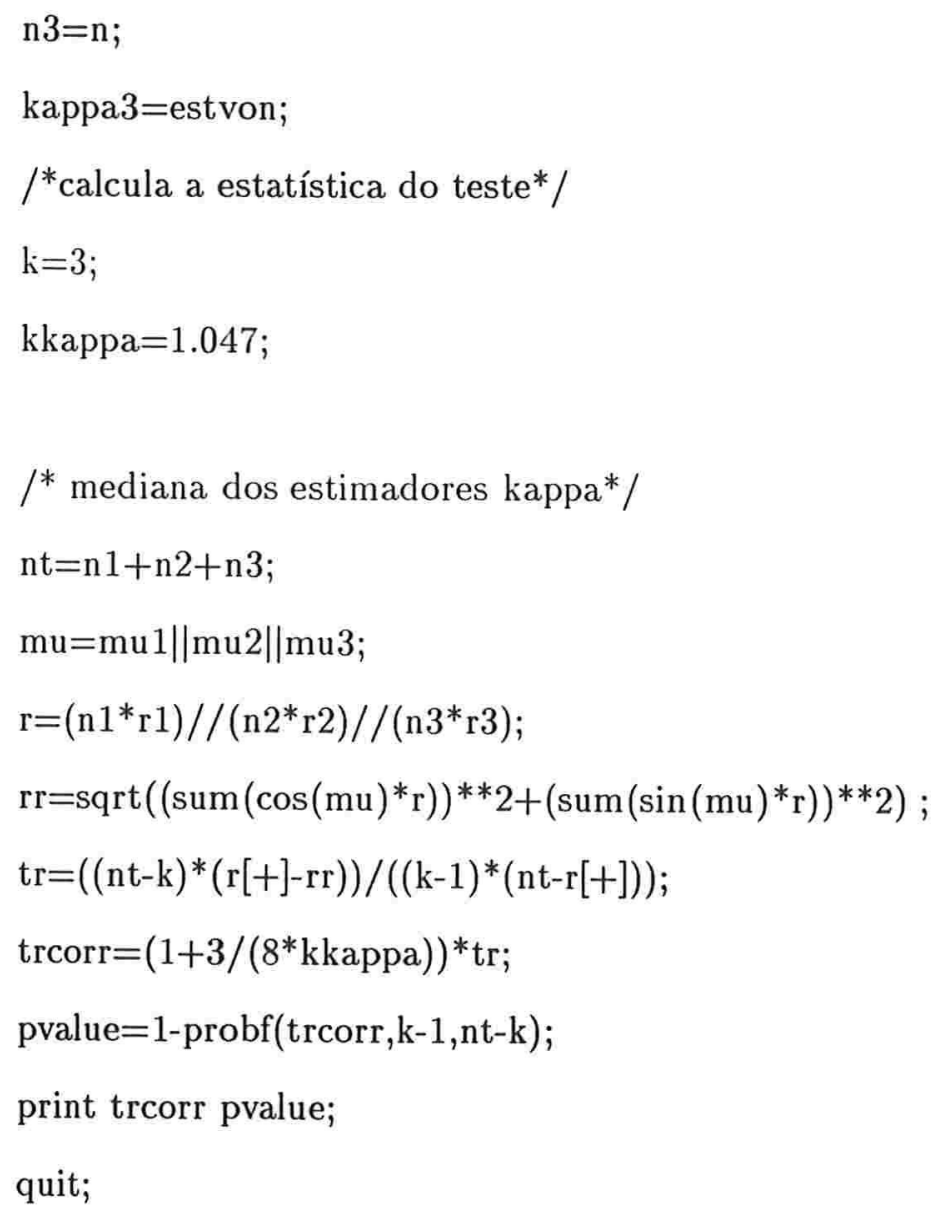

Módulo que gera amostras de tamanho $\mathrm{n}$ da distribuição de von Mises com parâmetros $\mu$ e $\kappa$ (em radianos).

start von;

$\mathrm{pi}=3.14159 ;$

$\mathrm{mu}=$ amedio* ${ }^{*} \mathrm{i} / 180$

$\mathrm{a}=1+\left(1+4^{*}\left(\right.\right.$ kappa\#\#2))\#\#0.5; $\mathrm{b}=\left(\mathrm{a}-\left(2^{*} \mathrm{a}\right) \# \# 0.5\right) /(2 *$ kappa $)$;

$\mathrm{r}=(1+\mathrm{b} \# \# 2) /(2 * \mathrm{~b})$;

$\mathrm{u} 1=\mathrm{j}(\mathrm{n}, 1) ; \mathrm{u} 2=\mathrm{j}(\mathrm{n}, 1) ; \mathrm{u} 3=\mathrm{j}(\mathrm{n}, 1)$; 
$\mathrm{z}=\mathrm{u} 1 ; \mathrm{f}=\mathrm{u} 1 ; \mathrm{c}=\mathrm{u} 1 ;$ teta $=\mathrm{u} 1 ;$ teta $1=\mathrm{u} 1 ;$

do $j=1$ to $n$;

p1:

$\mathrm{u} 1[\mathrm{j}]=\operatorname{ranuni}(2344)$;

$\mathrm{u} 2[\mathrm{j}]=\operatorname{ranuni}(1344)$;

$\mathrm{u} 3[\mathrm{j}]=\operatorname{ranuni}(26781234)$;

$\mathrm{z}[\mathrm{j}]=\cos \left(\mathrm{pi}^{*} \mathrm{u} 1[\mathrm{j}]\right)$;

$\mathrm{f}[\mathrm{j}]=\left(1+\mathrm{r}^{*} \mathrm{z}[\mathrm{j}]\right) /(\mathrm{r}+\mathrm{z}[\mathrm{j}])$;

$c[\mathrm{j}]=\operatorname{kappa}^{*}(\mathrm{r}-\mathrm{f}[\mathrm{j}])$;

if $c[j]^{*}(2-c[j])>u 2[j]$ then goto $p 2$;

else if $\log (c[j] / u 2[j])<(c[j]-1)$ then goto $p 1$;

else goto $\mathrm{p} 2$;

$\mathrm{p} 2: \operatorname{teta}[\mathrm{j}]=\bmod \left((\operatorname{sign}(\mathrm{u} 3[\mathrm{j}]-0.5) * \operatorname{arcos}(\mathrm{f}[\mathrm{j}])+\mathrm{mu}), 2^{*} \mathrm{pi}\right)$;

if teta[j] $<0$ then $\operatorname{teta} 1[\mathrm{j}]=\operatorname{teta}[\mathrm{j}]+2 * \mathrm{pi}$;

else teta $1[\mathrm{j}]=\operatorname{teta}[\mathrm{j}]$;

end;

tetag $1=$ teta $1 * 180 / \mathrm{pi} ; \quad /{ }^{*}$ dados em graus $* /$

print teta 1 tetag 1 ;

finish; 


\section{Bibliografia}

[1] Abramowitz, M. e Stegun, I. A. (1970). Handbook of mMathematical functions. New York: National Bureau of Standars. $(50,51)$

[2] Artes, R. (1997). Extensões da teoria das equações de estimação generalizados a dados circulares e modelos de dispersão . Tese de Doutorado, IME USP.

[3] Batschelet, E. (1981).Circular Statistics in Biology Data. London: Academic Press.

[4] Best, D. J. e Fisher, N. I. (1979). Efficient simulation of the von Mises distribution. Appl. Statist. 24 152-57.

[5] Cabrera, J., Schmidt-Koenig, K. e Watson, G.S. (1991). The statistical analysis of circular data. Chapter 10 (pp. 285-305) in Human Understanding and animal Awareness, Editors P.P.G. Bateson e P.H. Klopfer. Perspectives in Ethology Series, Volume 9. New York: Plenum Presss.

[6] Collet, D. (1980). Outliers in circular data. Appl. Statist.29, 50-57.

[7] Conover, W.J. (1971) Practical nonparametric Statistics. Wiley, New York and London.

[8] Fisher, N. I. (1993). Statistical analysis of circular data.Cambridge: Cambridge University Press.

[9] Fisher, N. I. (1986). Robust comparasion of dispersion for samples of direction data. Aust. J. Statist. 28, 213-219.

[10] Fisher, N. I. e Hall, P. G.(1989). Bootstrap confidence regions for directional data. $J$. Am. Statist. Assoc. 84, 996-1002.

[11] Fisher, N. I. e Hall, P. G.(1991). Bootstrap algoritms for small samples. J. Statist. Plan. Inference. 27,157-69.

[12] Fisher, N. I. e Lewis, T. (1983). Estimating the common mean direction of several circular or spherical distribution whit, differing dispersions. Biometrika 70, 333-41. 
[13] Fisher, N.I e Powell, C. McA. (1989). Statistical analysis of two-dimensional palaeocurrent data: Methods and Examples. Aust. J. Earth Sci. 36, 91-107.

[14] Hall, P. G. (1992). The bootstrap and Edgeworth Expansion. New York: Springer.

[15] Harrison, D., Kanji, R.J. (1986). Analysis of variance for circular data. J. Appl. Statist. 13, 123-138.

[16] Harrison, D., Kanji, R.J. (1988). The development of analysis of variace for circular data. J. Appl. Statist. 15, 197-224.

[17] Lockhart, R. A. E Stephens, M. A. (1985). Tests of fit for the von Mises distribution. Biometrika 72, 647-52.

[18] Mardia, K. V. (1967). A nonparametric test for the bivariate two-sample location problem. J. Roy. Stat. Soc. B 29, 320-342.

[19] Mardia, K. V. (1969). On the null distribution of a non-parametric test for the bivariate two-sample problem. J. Roy. Stat. Soc. B 31 98-102.

[20] Mardia, K. V. (1972). Statistics of Directional Data. London: Academic Press.

[21] Mardia, K. V. (1975). Statistics of directional data. (With Discusion). J. R. Statist. Soc. B 37,349-93.

[22] Neter, J. ; Kuter, M.H. ; Nachtsheim, C.J. Wasserman, W. (1996). Applied linear statistical models. London; Irwin.1

[23] Rao, J. S. (1966). Large sample tests for homogeneity of angular data. Appendix to a paper by S. Sengupta and J. S. Rao. Sankhya, Ser. B, 28, 172-174.

[24] Rao, J. S. (1984). Nonparametric methods in directional data. Chapter 31 in Handbook of Statistics, Volume 4. Nonparamtric Statistics, Editors P. R. Krishnaiah e P.K. Sen, pp. 755-70. Amsterdam: Elsevier Science Publishers.

[25] Stephens, M. A. (1969). Tests for the von Mises distribution. Biometrika 56, 149-60.

[26] Stephens, M. A. (1982). Use of the von Mises distribution to analyse continuos proportions. Biometrika 69, 730-7.

[27] Underwood, A.J. e Chapman, M.J. (1985). Multifactorial Analysis of Directional of Movement of Animals. Journal of Experimental Marine Biology an Ecology, 91, 17-43.

[28] Upton, G. J. G. (1973). Single-sample tests for the von Mises distribution. Biometrika 60, 87-99.

[29] Upton, G. J. G. (1986). Approximate confidence intervals for the mean direction of a von Mises distribution. Biometrika 73, 525-7. 
[30] Upton, G. J. G. e Fingleton, B. (1989). Spatial data analysis by example. Categorical and Directional data Volume 2. New York: John Wiley.

[31] Watson, G.S. (1983). Statistics on spheres. New York: Wiley.

[32] Wheeler, S. e Watson, G. S. (1964). A distribution-free two-samples test on a circle. Biometrika 51, 256-257.

[33] Zar, J. H. (1974). Biostatistical analysis. Prentice-Hall, Englewood Cliffs, N. J. 